\title{
2005s-04 \\ Distribution-Free Bounds for Serial Correlation Coefficients in Heteroskedastic Symmetric Time Series
}

\author{
Jean-Marie Dufour, Abdeljelil Farhat, \\ Marc Hallin
}

\begin{tabular}{c}
\hline Série Scientifique \\
Scientific Series
\end{tabular}

\author{
Montréal \\ Juillet 2004 \\ (publié en février 2005)
}

(C) 2005 Jean-Marie Dufour, Abdeljelil Farhat, Marc Hallin. Tous droits réservés. All rights reserved. Reproduction partielle permise avec citation du document source, incluant la notice (C)

Short sections may be quoted without explicit permission, iffull credit, including (C) notice, is given to the source.
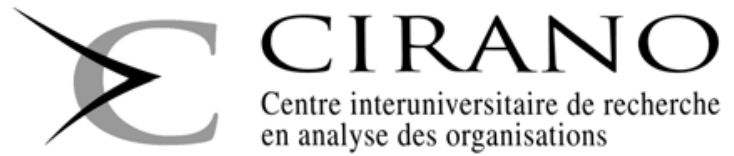

Centre interuniversitaire de recherche en analyse des organisations 


\section{CIRANO}

Le CIRANO est un organisme sans but lucratif constitué en vertu de la Loi des compagnies du Québec. Le financement de son infrastructure et de ses activités de recherche provient des cotisations de ses organisations-membres, d'une subvention d'infrastructure du Ministère du Développement économique et régional et de la Recherche, de même que des subventions et mandats obtenus par ses équipes de recherche.

CIRANO is a private non-profit organization incorporated under the Québec Companies Act. Its infrastructure and research activities are funded through fees paid by member organizations, an infrastructure grant from the Ministère du Développement économique et régional et de la Recherche, and grants and research mandates obtained by its research teams.

PARTENAIRE MAJEUR

$$
\text { Les organisations-partenaires / The Partner Organizations }
$$

. Ministère du Développement économique et régional et de la Recherche [MDERR]

PARTENAIRES

. Alcan inc.

. Axa Canada

. Banque du Canada

. Banque Laurentienne du Canada

. Banque Nationale du Canada

. Banque Royale du Canada

. Bell Canada

. BMO Groupe Financier

. Bombardier

. Bourse de Montréal

. Caisse de dépôt et placement du Québec

. Développement des ressources humaines Canada [DRHC]

. Fédération des caisses Desjardins du Québec

. GazMétro

. Groupe financier Norshield

. Hydro-Québec

. Industrie Canada

. Ministère des Finances du Québec

. Pratt \& Whitney Canada Inc.

. Raymond Chabot Grant Thornton

. Ville de Montréal

. École Polytechnique de Montréal

. HEC Montréal

. Université Concordia

. Université de Montréal

. Université du Québec

. Université du Québec à Montréal

. Université Laval

. Université McGill

. Université de Sherbrooke

ASSOCIE A :

. Institut de Finance Mathématique de Montréal (IFM²)

. Laboratoires universitaires Bell Canada

. Réseau de calcul et de modélisation mathématique $\left[\mathrm{RCM}^{2}\right]$

. Réseau de centres d'excellence MITACS (Les mathématiques des technologies de l'information et des systèmes complexes)

Les cahiers de la série scientifique $(\mathrm{CS})$ visent à rendre accessibles des résultats de recherche effectuée au CIRANO afin de susciter échanges et commentaires. Ces cahiers sont écrits dans le style des publications scientifiques. Les idées et les opinions émises sont sous l'unique responsabilité des auteurs et ne représentent pas nécessairement les positions du CIRANO ou de ses partenaires.

This paper presents research carried out at CIRANO and aims at encouraging discussion and comment. The observations and viewpoints expressed are the sole responsibility of the authors. They do not necessarily represent positions of CIRANO or its partners. 


\title{
Distribution-Free Bounds for Serial Correlation Coefficients in Heteroskedastic Symmetric Time Series"
}

\author{
Jean-Marie Dufour ${ }^{\dagger}$, Abdeljelil Farhat ${ }^{\ddagger}$ Marc Hallin ${ }^{\S}$
}

\begin{abstract}
Résumé / Abstract
Nous étudions le problème qui consiste à tester l'hypothèse que des observations $X_{1,} \cdots, X_{n}$ d'une série chronologique sont indépendantes avec des distributions non spécifiées (possiblement non identiques) symétriques autour d'une médiane connue. Nous proposons plusieurs bornes sur les distributions des coefficients d'autocorrélation : bornes exponentielles, bornes de type Eaton, bornes de Chebyshev et bornes de Berry-Esséen-Zolotarev. Les bornes sont exactes dans les échantillons finis, non paramétriques et faciles à calculer. Nous évaluons par simulation la performance des bornes et comparons celle-ci à celle de tests d'autocorrélation traditionnels. Les procédures proposées sont appliquées à des données de taux d'intérêt américaines ("commercial paper rate").
\end{abstract}

Mots clés : autocorrelation; serial dependence; nonparametric test; distribution-free test; heterogeneity; heteroskedasticity; symmetric distribution; robustness; exact test; bound; exponential bound; large deviations; Chebyshev inequality; Berry-Esséen; interest rates.

\footnotetext{
* The authors thank two anonymous referees and the Editor, Ronald Gallant, for several useful comments, as well as Sophie Mahseredjian for programming assistance. This work was supported by the the Belgian Federal Government (P.A.I. contract), the Canada Research Chair Program (Chair in Econometrics, Université de Montréal), the Institut de Finance mathématique de Montréal (IFM2), the Canadian Network of Centres of Excellence [program on Mathematics of Information Technology and Complex Systems, (MITACS)], the Communauté française de Belgique (A.R.C. contract), the Intercollegiate Center for Management Sciences (Bruxelles), the Fonds de recherche sur la société et la culture (Québec), the Fonds de recherche sur la nature et les technologies (Québec), the Natural Sciences and Engineering Research Council of Canada, the Social Sciences and Humanities Research Council of Canada, the Canada Council for the Arts (Killam Fellowship), and the Alexander-vonHumboldt Foundation (Germany).

${ }^{\dagger}$ Canada Research Chair Holder (Econometrics). Centre interuniversitaire de recherche en analyse des organisations (CIRANO), Centre interuniversitaire de recherche en économie quantitative (CIREQ), and Département de sciences économiques, Université de Montréal. Mailing address: Département de sciences économiques, Université de Montréal, C.P. 6128 succursale Centre-ville, Montréal, Québec, Canada H3C 3J7. Tel: 1 (514) 343-2400; Fax: 1 (514) 343-5831; e-mail: jean.marie.dufour@umontreal.ca. Web page: http://www.fas.umontreal.ca/SCECO/Dufour

ॠ Canada Research Chair in Econometrics, CIRANO and CIREQ, Université de Montréal, and Faculté des Sciences Économiques et de Gestion de Mahdia. Mailing address: Département des Méthodes Quantitatives, Faculté des Sciences Économiques et de Gestion de Mahdia, Sidi Messaoud Hiboun, Mahdia 5111, Tunisie. Tel: 21673 671190; Fax: 21673 671188; e-mail: abdeljelil.farhat@umontreal.ca.

${ }_{\S}^{\S}$ Département de mathématiques, I.S.R.O., and European Centre for Advanced Research in Economics and Statistics (ECARES), Université Libre de Bruxelles. Mailing address: ECARES, Université Libre de Bruxelles, Campus du Solbosch, CP 114, avenue F.D. Roosevelt 50, 1050 Bruxelles, Belgium. Tel: 322650 2886; Fax: 322650 5899; e-mail: mhallin@ulb.ac.be.
} 
We consider the problem of testing whether the observations $X_{1}, \cdots, X_{n}$ of a time series are independent with unspecified (possibly nonidentical) distributions symmetric about a common known median. Various bounds on the distributions of serial correlation coefficients are proposed: exponential bounds, Eaton-type bounds, Chebyshev bounds and Berry-EsséenZolotarev bounds. The bounds are exact in finite samples, distribution-free and easy to compute. The performance of the bounds is evaluated and compared with traditional serial dependence tests in a simulation experiment. The procedures proposed are applied to U.S. data on interest rates (commercial paper rate).

Keywords: autocorrelation; serial dependence; nonparametric test; distribution-free test; heterogeneity; heteroskedasticity; symmetric distribution; robustness; exact test; bound; exponential bound; large deviations; Chebyshev inequality; Berry-Esséen; interest rates.

Codes JEL : C14, C22, C12, C32, E4 


\section{Contents}

List of Propositions $\quad$ iv

$\begin{array}{ll}\text { 1. Introduction } & 1\end{array}$

2. Exponential bounds $\quad 3$

3. Bounds based on moments 5

4. Berry-Esséen-Zolotarev bounds 6

$\begin{array}{lll}\text { 5. Simulation experiment } & 7\end{array}$

6. Application to commercial paper rate 13

$\begin{array}{lll}\text { 7. Conclusion } & 16\end{array}$

$\begin{array}{ll}\text { A. Appendix: Proofs } & 17\end{array}$

B. Appendix: Conditional moments of the autocorrelations 18

C. Appendix: Additional simulation results $\quad 18$ 


\section{List of Propositions}

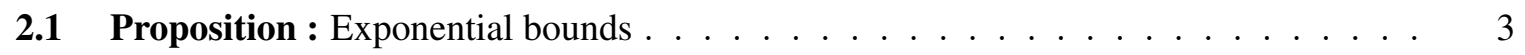

3.1 Proposition : Improved Eaton-Pinelis bounds . . . . . . . . . . . . . . 5

3.2 Proposition : Generalized Chebyshev bounds . . . . . . . . . . . . . 6

4.1 Proposition : Berry-Esséen-Zolotarev bounds . . . . . . . . . . . . . 7

Proof of Proposition $\mathbf{2 . 1} \ldots \ldots \ldots \ldots$

Proof of Proposition $\mathbf{3 . 1} \ldots \ldots \ldots \ldots$

Proof of Proposition $3.2 \ldots \ldots \ldots \ldots$

Proof of Proposition $4.1 \ldots \ldots \ldots$

\section{List of Tables}

1 Heterokedasticity patterns studied . . . . . . . . . . . . . . . 8

2 Empirical levels of serial dependence tests at nominal level $0.05 \ldots \ldots$. . . . . . . 9

3 Empirical powers of serial dependence tests at level $0.05 \ldots \ldots$. . . . . . . 10

4 Empirical powers of serial dependence tests at level $0.05 \ldots \ldots . . \ldots 11$

5 Quarterly Commercial Paper Interest Rate 1951:2-1983:4 (logarithm, first differences): autocorrelations and bounds on p-values for two-sided tests . . . . . . . . 14

6 Quarterly Commercial Paper Interest Rate 1951:2-1983:4 (logarithm, second differences) : autocorrelations and bounds on p-values for two-sided tests . . . . . 15

C.1 Empirical levels of serial dependence tests at nominal level 0.05. $n=30 \ldots \ldots$

C.2 Empirical levels of serial dependence tests at nominal level 0.05. $n=60 \ldots \ldots 20$

C.3 Empirical levels of serial dependence tests at nominal level 0.05. $n=100 \ldots \ldots \quad 21$

C.4 Critical values for size-corrected tests with level $0.05 \ldots \ldots \ldots$. . . . . . 22

C.5 Empirical powers of serial dependence tests at level $0.05, \varphi=0.2$, Gaussian errors 23

C.6 Empirical powers of serial dependence tests at level $0.05, \varphi=0.2$, Cauchy errors 24

C.7 Empirical powers of serial dependence tests at level 0.05, $\varphi=0.3$, Gaussian errors 25

C.8 Empirical powers of serial dependence tests at level 0.05, $\varphi=0.3$, Cauchy errors 26

C.9 Empirical powers of serial dependence tests at level 0.05, $\varphi=0.4$, Gaussian errors 27

C.10 Empirical powers of serial dependence tests at level 0.05, $\varphi=0.4$, Cauchy errors 28

C.11 Empirical powers of serial dependence tests at level $0.05, \varphi=0.5$, Gaussian errors 29

C.12 Empirical powers of serial dependence tests at level $0.05, \varphi=0.5$, Cauchy errors 30

C.13 Empirical powers of serial dependence tests at level 0.05, $\varphi=0.6$, Gaussian errors 31

C.14 Empirical powers of serial dependence tests at level $0.05, \varphi=0.6$, Cauchy errors 32

C.15 Empirical powers of serial dependence tests at level $0.05, \varphi=0.8$, Gaussian errors 33

C.16 Empirical powers of serial dependence tests at level $0.05, \varphi=0.8$, Cauchy errors 34

C.17 Empirical powers of serial dependence tests at level 0.05, $\varphi=0.9$, Gaussian errors 35

C.18 Empirical powers of serial dependence tests at level $0.05, \varphi=0.9$, Cauchy errors 36 


\section{Introduction}

Let $X_{1}, X_{2}, \ldots, X_{n}$ be a time series of length $n$. In many situations, it is of interest to test whether the $X_{t}$ 's are independent against an alternative of serial dependence, say, at lag $k(k \geq 1)$. If under the null hypothesis the observations are assumed to be identically distributed with known mean $\mu$, a natural test consists in rejecting the null hypothesis for large or small values of the autocorrelation coefficient

$$
r_{k}=\sum_{t=1}^{n-k}\left(X_{t}-\mu\right)\left(X_{t+k}-\mu\right) / \sum_{t=1}^{n}(X-\mu)^{2}
$$

where $1 \leq k \leq n-1$. Under general regularity conditions, the distribution of $r_{k}$ is approximately normal with mean zero and variance $n^{-1}$; see Anderson (1971, chapter 8) or Brockwell and Davis (1991, chapter 7).

When the observations are not identically distributed or their distributions are heavy-tailed, such a procedure can clearly be inappropriate. In this paper, we study the null hypothesis $H_{0}$ under which the observations $X_{1}, \ldots, X_{n}$ are independent but possibly nonidentically distributed, with distributions symmetric about known medians $\mu_{t}$. No assumption about the existence of the moments of $X_{1}, \ldots, X_{n}$ is made, and the distribution of the observations can be discrete. Since $X_{t}$ can be replaced by $X_{t}-\mu_{t}$, we can, without loss of generality, assume that $\mu_{1}=\cdots=\mu_{n}=0$. Consequently, we shall henceforth set $\mu_{t}=0, t=1, \ldots, n$.

The hypothesis $H_{0}$ is "nonparametric" in the sense that no finite-dimensional parameter vector can determine entirely the probability distribution of the observations $X_{1}, X_{2}, \ldots, X_{n}$. Following standard terminology [see Lehmann (1986, sections 3.1 and 3.5)], a test of $H_{0}$ has level $\alpha$ if the probability of rejecting $H_{0}$ is not greater than $\alpha$ under any distribution of $X=\left(X_{1}, \ldots, X_{n}\right)^{\prime}$ included in $H_{0}(0<\alpha<1)$. If moreover the supremum of the rejection probabilities over $H_{0}$ is equal to $\alpha$, one says that the test has size $\alpha$. Since $H_{0}$ covers a wide spectrum of probability distributions and because of the "parametric origin" of the coefficient $r_{k}$, the distribution of $r_{k}$ under $H_{0}$ depends on the form of the distribution of the observations. Without additional assumptions, it is unknown. Consequently, no similar critical region of the type $\left|r_{k}\right|>c$ (where $c$ is a nonstochastic critical point which depends on the level of the test) does exist: i.e., for $0<c<1$, the probability of the event $\left|r_{k}\right|>c$ is not constant over the set of data generating processes (DGP) in $H_{0}$, and finding a valid critical value involves bounding the distribution of $r_{k}$ over $H_{0}$ or considering data-dependent critical regions for $r_{k}$. In particular, there is strictly no guarantee that the actual sizes of tests based on the asymptotic (normal) distribution of $r_{k}$ will be less than or equal to their nominal level (as tests of $H_{0}$ ) in finite samples. The same will hold a fortiori for critical values obtained under parametric assumptions, e.g., the assumption that $X_{1}, \ldots, X_{n}$ are independent and identically distributed (i.i.d.) random variables according to a $\mathrm{N}\left(0, \sigma^{2}\right)$ distribution [in which case exact critical values may be computed using Imhof's algorithm]: such critical values - though they belong to daily practice - simply do not yield valid tests of the nonparametric hypothesis $H_{0}$.

The objective of this paper is to develop finite-sample ( $\alpha$-level) tests based on $r_{k}$ for the nonparametric null hypothesis $H_{0}$. In other words, we need to ensure that the probability of rejecting $H_{0}$ is not greater than $\alpha$ under any DGP in $H_{0}$. This problem is quite distinct from the one where one tries to approximate the distribution of $r_{k}$ under some specific distribution included in $H_{0}$ (like 
the i.i.d. Gaussian model). Following a classical nonparametric technique, we shall do this here by using an appropriate conditioning. When $X_{1}, X_{2}, \ldots, X_{n}$ are absolutely continuous, the vector of absolute values $|X|=\left(\left|X_{1}\right|, \ldots,\left|X_{n}\right|\right)^{\prime}$ is a complete sufficient statistic for $H_{0}$. Further, classical arguments of similarity and Neyman structure lead one to consider tests that are conditional with respect to the complete sufficient statistic $|X|$; see Lehmann (1986, chapter 4). Indeed, conditioning on $|X|$ is a necessary requirement to obtain a valid test under conditions of general heterogeneity (heteroskedasticity); see Lehmann and Stein (1949), Pratt and Gibbons (1981, Section 5.10), Dufour and Hallin (1991, section 1), and Dufour (2003, section 4.2)]. The conditional distribution of $X=\left(X_{1}, X_{2}, \ldots, X_{n}\right)^{\prime}$ given $|X|$ is then determined by the distribution of the signs of $X_{1}, \ldots, X_{n}$. Since, under $H_{0}$, the signs are independent symmetric Bernoulli variables, the conditional distribution of $r_{k}$ (given the vector of absolute values $|X|$ ) may in principle be computed, e.g., by enumeration. In practice, however, the conditional distribution of $r_{k}$ depends on each specific sample, because it is a function of $|X|$, and so finding critical values may be difficult. This problem is also met in the well-known case of permutation $t$-tests; see Pratt and Gibbons (1981, chapter 4).

For the problem of testing $H_{0}$ against location-shift alternatives, simple bounds for the conditional and unconditional distributions of the $t$-statistic were provided in Edelman $(1986,1990)$ and Dufour and Hallin (1991, 1993); similar bounds for general linear signed rank statistics have also been proposed in Dufour and Hallin (1992). Beyond the important advantage of exactness for any sample size, extensive comparisons in Dufour and Hallin $(1991,1992,1993)$ indicate that the bounds studied (exponential, Chebyshev-type, Eaton-type, Berry-Esséen) can be surprisingly tight, especially if one takes the minimum of the various bounds.

In this paper, we give analogous results for tests of $H_{0}$ based on $r_{k}$ against serial dependence alternatives. Four types of bounds are presented: (1) exponential bounds (Proposition 2.1); (2) improved Eaton bounds (Proposition 3.1); (3) Chebyshev-type bounds (Proposition 3.2); (4) BerryEsséen-Zolotarev bounds (Proposition 4.1). The exponential bounds are based on the conditional moment generating function of $r_{k}$ (given $|X|$ ), the improved Eaton and Chebyshev-type bounds on conditional moments of $r_{k}$ (a truncated third moment in the case of the Eaton bound), while the Berry-Esséen-Zolotarev bound is based on the normal distribution function. The exponential, Eaton, Chebyshev and Berry-Esséen bounds extend to the case of autocorrelation coefficients the bounds proposed in Dufour and Hallin (1991, 1992, 1993).

All these bounds are exact in finite samples and simple to compute. They are applicable despite the presence of general forms of nonnormality and heteroskedasticity (provided the symmetry hypothesis holds). In particular, no assumption on the existence of moments is required, and the variables considered may have continuous or discrete distributions. None of the bounds given uniformly dominates the others. While the three first classes of bounds are especially useful to obtain upper bounds for small tail areas, the Berry-Esséen bounds can be tighter for larger tail areas (i.e., tails associated with points that are closer to the center of the distribution) and yield lower bounds on tail areas as well. Conservative conditional (given $|X|$ ) as well as unconditional conservative $p$-values, or critical points, for tests based on $r_{k}$ can be obtained from any one of these bounds. Since all the bounds are simple to compute, the obvious strategy here is to take the smallest $p$-value yielded by the different bounds (or, equivalently, the tightest critical point). Such $p$-values provide 
a useful nonparametric check on the significance of tests based on autocorrelation coefficients.

The exponential bounds are described in section 2, the Eaton and Chebyshev bounds are given in section 3, while the Berry-Esséen bounds are derived in section 4. In section 5, simulation results on the performance of the bounds are presented. In section 6, we illustrate the use of the bounds by applying them to data on commercial paper interest rates in the U.S. We conclude in section 7.

\section{Exponential bounds}

In the following proposition, we derive exponential bounds for the tail areas of the conditional distribution of $r_{k}$ given $|X|$ under the null hypothesis that $X_{1}, \ldots, X_{n}$ are independent with distributions symmetric about zero. The notation a.s. means almost surely, while the symbol ":=" represents a definition. The proofs of the propositions appear in appendix A.

Proposition 2.1 Exponential bounds. Let $X_{1}, \ldots, X_{n}$ be independent random variables with distributions symmetric about zero, $|X|=\left(\left|X_{1}\right|, \ldots,\left|X_{n}\right|\right)^{\prime}$, and

$$
\begin{aligned}
r_{k} & :=\sum_{t=1}^{n-k} X_{t} X_{t+k} / \sum_{t=1}^{n} X_{t}^{2}, \quad 1 \leq k \leq n-1, \\
w_{k t} & :=\left|X_{t} X_{t+k}\right| /\left(\sum_{\tau=1}^{n-k} X_{\tau}^{2} X_{\tau+k}^{2}\right)^{1 / 2}, \quad t=1, \ldots, n-k,
\end{aligned}
$$

where we use the convention $0 / 0=0$. Then the conditional distribution of $r_{k}$ given $|X|$ is symmetric about zero and

$$
\begin{aligned}
\mathrm{P}\left[r_{k} \geq y|| X \mid\right] & \leq B_{k}\left(y_{k},|X|\right) \leq \exp \left(-y_{k}^{2}\right) \prod_{t=1}^{n-k} \cosh \left(w_{k t} y_{k}\right) \\
& \leq \exp \left(-y_{k}^{2}\right)\left[\cosh \left(y_{k} / \sqrt{n_{k}^{*}}\right)\right]^{n_{k}^{*}} \leq \exp \left(-y_{k}^{2} / 2\right)
\end{aligned}
$$

a.s., for all $y>0$ and $1 \leq k \leq n-1$, where $y_{k}:=y / D_{k}(|X|), \cosh (x):=\left(e^{x}+e^{-x}\right) / 2$, $n_{k}^{*}:=\operatorname{card}\left(\left\{t:\left|X_{t} X_{t+k}\right| \neq 0,1 \leq t \leq n-k\right\}\right)$ is the number of products $X_{t} X_{t+k}$ different from zero,

$$
\begin{aligned}
D_{k}(|X|) & :=\left(\sum_{t=1}^{n-k} X_{t}^{2} X_{t+k}^{2}\right)^{1 / 2} /\left(\sum_{t=1}^{n} X_{t}^{2}\right), \\
B_{k}(y,|X|) & :=\inf _{z \geq 0}\left\{\exp (-z y) \prod_{t=1}^{n-k} \cosh \left(w_{k t} z\right)\right\}
\end{aligned}
$$

and the four bounds in (2.3) are set equal to zero when $D_{k}(|X|)=0$. 
From the symmetry of the conditional distribution of $r_{k}$, it is clear that $\mathrm{P}\left[\left|r_{k}\right| \geq y|| X \mid\right]=$ $2 \mathrm{P}\left[r_{k} \geq y|| X \mid\right]=2 \mathrm{P}\left[r_{k} \leq-y|| X \mid\right]$ a.s., so that (2.3) can also be used to bound $\mathrm{P}\left[r_{k} \leq-y\right]$ and $\mathrm{P}\left[\left|r_{k}\right| \geq y|| X \mid\right]$ for any $y>0$. In (2.3), four bounds on the tail areas $\mathrm{P}\left[r_{k} \geq y|| X \mid\right]$ are given. Denote them by $E_{1 k} \leq E_{2 k} \leq E_{3 k} \leq E_{4 k}$ in ascending order. These bounds are increasingly looser, but the larger ones are easier to compute. In particular, $E_{2 k}, E_{3 k}$ and $E_{4 k}$ only require information about the second empirical moments of the sample $\left(r_{k}\right.$ and $\left.\sum X_{t}^{2}\right)$, which may be useful when the complete observation vector $X=\left(X_{1}, \ldots, X_{n}\right)^{\prime}$ is not available to an investigator. The exponential bound $E_{4 k}=\exp \left(-y_{k}^{2} / 2\right)$ is similar to a bound given by Edelman (1986) and Efron (1969) for the case of $t$-statistics; for an earlier related result, see also Hoeffding (1963). In contrast with the case of $t$-statistics, however, this bound now explicitly depends on $|X|$ through $D_{k}(|X|)$. The second largest bound $E_{3 k}=\exp \left(-y_{k}^{2}\right)\left[\cosh \left(y_{k} / \sqrt{n_{k}^{*}}\right)\right]^{n_{k}^{*}}$ uniformly improves the latter by explicitly taking into account the sample size and the lag. It is based on a result given by Eaton (1970) for linear combinations of independent Bernoulli variables. For example, for $n-k=10$ and $y_{k}=3$, we have $E_{3 k}=0.0064$ while $E_{4 k}=0.0111$. Similarly, the bound $E_{2 k}=\exp \left(-y_{k}^{2}\right)$ $\prod_{t=1}^{n-k} \cosh \left(w_{k t} y_{k}\right)$ improves the two previous ones by explicitly taking into account the weights $w_{k t}, t=1, \ldots, n-k$. When the weights are equal, i.e., $w_{k t}=1 / \sqrt{n_{k}^{*}}, t=1, \ldots, n-k$, the bounds $E_{2 k}$ and $E_{3 k}$ coincide. In the other cases, $E_{2 k}$ can yield substantial improvements over $E_{3 k}$, especially when the data contain a large outlier. For example, if $w_{k t} \rightarrow 0, n_{k}^{*}=10$ and $y_{k}=3$, the ratio $E_{2 k} / E_{3 k}$ converges to 0.1933 . Finally, the smallest bound $E_{1 k} \equiv B_{k}\left(y_{k},|X|\right)$ is obtained by finding the infimum of the function $M_{k}(z)=\exp \left(-z y_{k}\right) \prod_{t=1}^{n-k} \cosh \left(w_{k t} z\right)$ for $z \geq 0 . E_{4 k}$ in turn can yield substantial improvement over the previous bounds. The function $B_{k}(y,|X|)$ has the following more explicit expression:

$$
\begin{aligned}
B_{k}(y,|X|) & =0, & & \text { if } \sum_{t=1}^{n-k} w_{k t}<y, \\
& =\left(\frac{1}{2}\right)^{n_{k}^{*}}, & & \text { if } \sum_{t=1}^{n-k} w_{k t}=y, \\
& =\exp \left(-z_{k}^{*} y\right) \prod_{t=1}^{n-k} \cosh \left(w_{k t} z_{k}^{*}\right), & & \text { if } \sum_{t=1}^{n-k} w_{k t}>y,
\end{aligned}
$$

where $z_{k}^{*}$ is the unique positive number that solves the equation

$$
\sum_{t=1}^{n-k} w_{k t}\left[\left(1-e^{-2 w_{k t} z_{k}^{*}}\right) /\left(1+e^{-2 w_{k t} z_{k}^{*}}\right)\right]=y .
$$

It is fairly easy to compute $B_{k}(y,|X|)$ by numerical methods; for further discussion, see Dufour and Hallin (1992, pp. 315-317).

Since they depend on $|X|$ only through $D_{k}(|X|)$, the two largest bounds $E_{3 k}$ and $E_{4 k}$ in (2.3) also yield simple unconditional bounds: for all $y>0$,

$$
\mathrm{P}\left[r_{k} \geq y D_{k}(|X|)\right] \leq \exp \left(-y^{2}\right)[\cosh (y / \sqrt{n-k})]^{n-k} \leq \exp \left(-y^{2} / 2\right) .
$$


However, in most practical cases, the weights $w_{k t}$ are known so that the better bounds $E_{1 k}$ and $E_{2 k}$ are available: conditional critical values based on the latter always yield less conservative tests (both conditionally and unconditionally).

\section{Bounds based on moments}

The exponential bounds described in Proposition $\mathbf{2 . 1}$ are based on the conditional moment generating function of $r_{k}$ given $|X|$. In this section, we give two sets of bounds based on considering appropriate conditional moments of $r_{k}$. The first one applies results from Eaton (1970), Pinelis (1994) and Dufour and Hallin (1993), and is based on minimizing a truncated third order moment. We denote by $\varphi(y)=(2 \pi)^{-1 / 2} \exp \left(-y^{2} / 2\right)$ the $\mathrm{N}(0,1)$ density function, and by $(y)_{+}$the positive part of any real number $y$, i.e., $(y)_{+}=\max (0, y)$.

Proposition 3.1 Improved EATON-PINELIS BOUNDS. Under the assumptions and notations of Proposition 2.1, we have:

$$
\begin{aligned}
\mathrm{P}\left[r_{k} \geq y|| X \mid\right] & \leq \min \left\{B_{E}\left(y_{k} ; n_{k}^{*}\right), 0.5 y_{k}^{-2}, 0.5\right\}:=B_{E P}^{*}\left(y_{k} ; n_{k}^{*}\right) \\
& \leq \min \left\{B_{E}\left(y_{k}\right), 0.5 y_{k}^{-2}, 0.5\right\}:=B_{E P}\left(y_{k}\right)
\end{aligned}
$$

a.s., for all $y>0$, where

$$
\begin{aligned}
& B_{E}(y ; m):=(0.5) \inf _{0 \leq c<y}\left\{(0.5)^{m} \sum_{j=0}^{m}\left(\begin{array}{c}
m \\
j
\end{array}\right) f_{c}\left[(j-(m / 2)) /(m / 4)^{1 / 2}\right] /(y-c)^{3}\right\} \\
& f_{c}(x):=\left[(|x|-c)_{+}\right]^{3},\left(\begin{array}{c}
m \\
j
\end{array}\right):=m ! /[j !(m-j) !], \text { and } \\
& B_{E}(y):=\inf _{0 \leq c<y} \int_{c}^{\infty}\left(\frac{z-c}{y-c}\right)^{3} \varphi(z) d z \\
&=\inf _{0 \leq c<y}\left\{\left[\varphi(c)\left(2+c^{2}\right)-(1-\Phi(c))\left(c^{3}+3 c\right)\right] /(y-c)^{3}\right\}
\end{aligned}
$$

Calculation of the bounds, $B_{E P}^{*}(y ; m)$ and $B_{E P}(y)$ is discussed in Dufour and Hallin (1993), where the associated (conservative) critical values for standard significance levels are also reported. It is of interest to note that the bound $B_{E P}$ enjoys an optimality property in the sense that it is tightest among all bounds based on expectations of convex functions of a standard normal variable; see Pinelis (1994) and Dufour and Hallin (1993). Note also that the function $B_{E}(y ; m)$ is monotonic increasing in $m$, i.e., $B_{E}(y ; m) \leq B_{E}(y ; m+1)$ for $y>0$.

Another related method consists in bounding the tail areas of $r_{k}$ with Chebyshev-type inequalities. As observed in Dufour and Hallin (1992), such bounds can be quite tight, especially if they are based on higher-order moments (i.e., moments of order greater than 2). We summarize these in the following proposition. 
Proposition 3.2 Generalized Chebyshev bounds. Let the assumptions and notations of Proposition 2.1 hold. Then, for any positive even integer $p$ and for any $y>0$,

$$
\begin{aligned}
\mathrm{P}\left[r_{k} \geq y|| X \mid\right] & \leq \frac{\mathrm{E}\left(r_{k}^{p}|| X \mid\right)}{2 y^{p}} \leq \frac{D_{k}(|X|)^{p} \mathrm{E}\left[Y\left(n_{k}^{*}\right)^{p}\right]}{2 y^{p}} \\
& \leq\left[\frac{(p-1)(p-3) \cdots 3 \cdot 1}{2 y^{p}}\right] D_{k}(|X|)^{p}
\end{aligned}
$$

and

$$
\mathrm{P}\left[r_{k} \geq y|| X \mid\right] \leq\left[\frac{\left(p_{k}^{*}-1\right)\left(p_{k}^{*}-3\right) \cdots 3 \cdot 1}{2 y^{p_{k}^{*}}}\right] D_{k}(|X|)^{p_{k}^{*}}
$$

a.s., where $Y(m)$ refers to a $\operatorname{Bin}(m, 0.5)$ random variable, $p_{k}^{*}=\max \left\{2, \bar{p}_{k}\right\}$ and $\bar{p}_{k}$ is the largest even integer such that $\bar{p}_{k}<1+y_{k}^{2}$.

To implement the first bound in (3.4), we need the conditional moments of $r_{k}$ given $|X|$. These can be established easily from (A.1), (A.2) and (A.3) in the proof of Proposition $\mathbf{2 . 1}$ and equations (3.2) to (3.6) in Dufour and Hallin (1992); the appropriate expressions are given in Appendix B. Even moments $\mathrm{E}\left[r_{k}^{p}|| X \mid\right]$ of order greater than 12 can be established by analogous methods, but the algebra is correspondingly more involved. These moments as well as well as higher order ones can also be established by using symbolic manipulation programs. The standardized binomial moments can be computed up to any desired order from formulae (3.8) and (3.9) in Dufour and Hallin (1992), and so the two larger bounds in (3.4) above can be obtained easily for any value of $p$. Clearly, the bounds in (3.4) can be computed for several values of $p$ and the minimum of these bounds again provides a valid bound. The bound (3.5) is the explicit solution of this minimization process (over all even values of $p \geq 2$ ) based on the third bound in (3.4), which is based on the moments of a $\mathrm{N}(0,1)$ distribution.

\section{Berry-Esséen-Zolotarev bounds}

The results of the two previous sections yield upper bounds on the tail areas of autocorrelation coefficients under the null hypothesis of independence, and they can therefore be used to check whether we can safely reject the null hypothesis at a given level under relatively weak nonparametric assumptions. Further, these bounds are reasonably tight only when $y$ is not too small (say, $y>1.5$ ). In many cases, it would also be helpful to have a lower bound which could be used to decide whether an autocorrelation coefficient unambiguously lies in the acceptance region of the (conditional) test based on $r_{k}$.

Unfortunately, it appears much more difficult to obtain lower bounds similar to the upper bounds previously given. In order to obtain such lower bounds as well as upper bounds whose behavior may be more satisfactory for lower values of $y$, we will consider bounds of the Berry-Esséen type. More precisely, in the following proposition, we combine results of van Beek (1972) and Zolotarev (1965) to bound the difference between the conditional distribution of $r_{k}$ and the standard normal one. 
Proposition 4.1 BerRy-EsséEn-Zolotarev bounds. Under the assumptions and notations of Proposition 2.1 and provided $X_{t} X_{t+k} \neq 0$ for at least one $t(1 \leq t \leq n-k)$, we have

$$
\begin{aligned}
\mid \mathrm{P}\left[r_{k} \geq y|| X \mid\right] & -\Phi\left[y / D_{k}(|X|)\right] \mid \\
& \leq \Delta:=\min \left\{0.7975 \sum_{t=1}^{n}\left|w_{k t}\right|^{3}, 0.366145\left(\sum_{t=1}^{n}\left|w_{k t}\right|^{3}\right)^{1 / 4}\right\} \\
& \leq 0.366145
\end{aligned}
$$

for all $y$, where $\Phi(y)$ denotes the $\mathrm{N}(0,1)$ distribution function.

It is clear that inequality (4.1) can provide both upper and lower bounds on the tail areas of $r_{k}$ :

$$
\begin{aligned}
B E_{L}:=1-\Phi\left[y / D_{k}(|X|)\right]-\Delta & \leq \mathrm{P}\left[r_{k} \geq y|| X \mid\right] \\
& \leq 1-\Phi\left[y / D_{k}(|X|)\right]+\Delta:=B E_{U} \quad \text { a.s. }
\end{aligned}
$$

This implies that the normal approximation is good when $\sum\left|w_{k t}\right|^{3}$ is small. It also follows from (4.1) that the conditional distribution of $r_{k}$ given $|X|$ - hence also its unconditional distribution converges to a normal distribution when $\sum\left|w_{k t}\right|^{3}$ goes to zero. But, of course, the main interest of (4.1) lies in the fact that it is an operational finite-sample approximation result, not a convergence theorem.

\section{Simulation experiment}

In order to provide some evidence on the size and power of the proposed bounds, we considered an $\mathrm{AR}(1)$ process of the form:

$$
\begin{aligned}
X_{t} & =\varphi X_{t-1}+u_{t}, \quad t=1, \ldots, n, \\
u_{t} & =d_{t} v_{t}, \quad t=1, \ldots, n,
\end{aligned}
$$

where the variables $v_{t}, t=1, \ldots, n$, are i.i.d., the $d_{t}$ 's are scale parameters which determine the form of the heteroskedasticity, and $X_{t}=0$ (fixed). Two types of distributions for $v_{t}$ were considered:

$$
\begin{array}{ll}
\text { (G) } \quad v_{t} \stackrel{i . i . d .}{\sim} \mathrm{N}(0,1), \quad t=1, \ldots, n, \\
\text { (C) } v_{t} \stackrel{i . i . d .}{\sim} \text { Cauchy, } \quad t=1, \ldots, n .
\end{array}
$$

For the error heterogeneity, the patterns described in Table 1 were studied.

Results of our simulation are reported in Tables 2 - 4. In these tables, the statistics $\left|t\left(r_{1}\right)\right|$, $\left|\tilde{t}\left(r_{1}\right)\right|,\left|t\left(\hat{\rho}_{k}\right)\right|$ and $\left|\bar{t}\left(\hat{\rho}_{k}\right)\right|$ represent four alternative ways of standardizing traditional (parametric) autocorrelation coefficients, while $E_{11}$ is the best exponential bound. The autocorrelation statistics 
Table 1. Heteroskedasticity patterns studied

\begin{tabular}{|c|c|c|c|c|}
\hline & Type & & & \\
\hline M1 & Homoskedasticity & $d_{t}=$ & 1, & $t=1, \ldots, n$ \\
\hline M2 & One outlier I & $\begin{aligned} d_{t} & = \\
& =\end{aligned}$ & $\begin{array}{l}10 \\
1\end{array}$ & $\begin{array}{l}\text { if } t=n / 2 \\
\text { otherwise }\end{array}$ \\
\hline M3 & One outlier II & $\begin{aligned} d_{t} & = \\
& =\end{aligned}$ & $\begin{array}{l}100, \\
1, \\
\end{array}$ & $\begin{array}{l}\text { if } t=n / 2 \\
\text { otherwise }\end{array}$ \\
\hline M4 & Exponential I & $d_{t}=$ & $e^{t / 10}$ & $t=1, \ldots, n$ \\
\hline M5 & Exponential II & $d_{t}=$ & $e^{t / 2}$, & $t=1, \ldots, n$ \\
\hline M6 & Two outliers I & $\begin{aligned} d_{t} & = \\
& =\end{aligned}$ & $\begin{array}{l}10 \\
1, \\
\end{array}$ & $\begin{array}{l}\text { if } t=\frac{n}{2} \text { or } \frac{n}{2}+1 \\
\text { otherwise }\end{array}$ \\
\hline M7 & Two outliers II & $\begin{aligned} d_{t} & = \\
& =\end{aligned}$ & $\begin{array}{l}100, \\
1,\end{array}$ & $\begin{array}{l}\text { if } t=\frac{n}{2} \text { or } \frac{n}{2}+1 \\
\text { otherwise }\end{array}$ \\
\hline M8 & Two outliers III & $\begin{aligned} d_{t} & = \\
& =\end{aligned}$ & $\begin{array}{l}10^{6}, \\
1,\end{array}$ & $\begin{array}{l}\text { if } t=\frac{n}{2} \text { or } \frac{n}{2}+1 \\
\text { otherwise }\end{array}$ \\
\hline
\end{tabular}

are:

$$
\begin{gathered}
\left|t\left(r_{k}\right)\right|=\left|\sqrt{n} r_{k}\right|, \quad\left|\tilde{t}\left(r_{k}\right)\right|=\left|r_{k} / \sigma_{k}\right|, \\
\left|t\left(\hat{\rho}_{k}\right)\right|=\left|\sqrt{n} \hat{\rho}_{k}\right|, \quad\left|\bar{t}\left(\hat{\rho}_{k}\right)\right|=\left|\left(\hat{\rho}_{k}-\mu_{k}\right) / \sigma_{k}\right|,
\end{gathered}
$$

where $r_{k}$ is defined in (2.1),

$$
\hat{\rho}_{k}=\sum_{t=1}^{n-k}\left(X_{t}-\bar{X}\right)\left(X_{t+k}-\bar{X}\right) / \sum_{t=1}^{n}\left(X_{t}-\bar{X}\right)^{2}
$$

is the usual "centered" autocorrelation coefficient, while $\mu_{k}=-(n-k) /[n(n+1)]$ and $\sigma_{k}^{2}=$ $(n-k) /[n(n+2)]$ are the adjusted mean and variance suggested in Dufour and Roy (1985) for the case of a sequence of i.i.d. observations.

For each of the above parametric statistics, we also report the results of tests based on four ways of computing critical values: (1) standard asymptotic normal critical values; (2) critical points based on the Imhof algorithm assuming the observations are i.i.d. Gaussian (the Imhof critical values were also cross-checked by simulation); (3) critical values obtained by simulation under each specific distribution and heteroskedasticity pattern considered; (4) critical values based on the largest critical point that we found over the set of distributions and heteroskedasticity patterns considered - i.e. models M1 - M8 with $v_{t}$ following a $\mathrm{N}(0,1)$ or a Cauchy distribution - which are all included in the null hypothesis of independence. Of course, the second method is the best choice under the assumptions made by the Imhof algorithm, but will not control the level (in the sense of 
Table 2. Empirical levels of serial dependence tests at nominal level $\alpha=0.05$

\begin{tabular}{|c|c|c|c|c|c|c|c|c|c|c|}
\hline \multirow{3}{*}{$\begin{array}{c}\text { Sample size: } n=30 \\
\text { Error distribution }\left(v_{t}\right) \\
\text { Heteroskedasticity type }\end{array}$} & \multicolumn{10}{|c|}{ Asymptotic tests and bounds } \\
\hline & \multicolumn{5}{|c|}{$\mathrm{N}(0,1)$} & \multicolumn{5}{|c|}{ Cauchy } \\
\hline & M1 & M2 & M5 & M7 & M8 & M1 & M2 & M5 & M7 & M8 \\
\hline$\left|t\left(r_{1}\right)\right|$ & 3.90 & 1.67 & 33.60 & 49.46 & 51.32 & 2.47 & 2.43 & 18.68 & 23.44 & 34.36 \\
\hline$\left|\tilde{t}\left(r_{1}\right)\right|$ & 4.96 & 2.20 & 36.17 & 52.26 & 54.42 & 2.95 & 2.88 & 20.34 & 25.86 & 36.92 \\
\hline$\left|t\left(\hat{\rho}_{k}\right)\right|$ & 4.22 & 1.91 & 31.78 & 47.29 & 49.11 & 2.43 & 2.32 & 17.58 & 21.91 & 32.69 \\
\hline$\left|\bar{t}\left(\hat{\rho}_{k}\right)\right|$ & 4.65 & 2.17 & 34.45 & 49.90 & 51.95 & 2.88 & 2.62 & 19.15 & 23.93 & 34.90 \\
\hline$E_{11}$ & 0.95 & 0.87 & 2.28 & 0.86 & 0.00 & 1.10 & 1.33 & 2.84 & 1.68 & 0.01 \\
\hline \multirow{2}{*}{ Best bound } & 1.11 & 1.00 & 2.28 & 0.87 & 0.00 & 1.16 & 1.36 & 2.84 & 1.68 & 0.01 \\
\hline & \multicolumn{10}{|c|}{ Tests based on Imhof critical values } \\
\hline$\left|t\left(r_{1}\right)\right|$ & 5.09 & 2.30 & 36.59 & 52.75 & 54.88 & 3.04 & 2.96 & 20.77 & 26.34 & 37.30 \\
\hline$\left|\tilde{t}\left(r_{1}\right)\right|$ & 5.45 & 2.49 & 37.39 & 53.71 & 55.65 & 3.21 & 3.17 & 21.41 & 27.13 & 37.97 \\
\hline$\left|t\left(\hat{\rho}_{k}\right)\right|$ & 5.57 & 2.53 & 35.01 & 50.98 & 52.37 & 2.95 & 3.10 & 19.91 & 24.78 & 35.58 \\
\hline \multirow[t]{2}{*}{$\left|\bar{t}\left(\hat{\rho}_{k}\right)\right|$} & 5.20 & 2.47 & 35.74 & 51.34 & 53.54 & 3.08 & 2.82 & 20.05 & 25.48 & 36.12 \\
\hline & \multicolumn{10}{|c|}{ Tests based on global size correction } \\
\hline$\left|t\left(r_{1}\right)\right|$ & 0.02 & 0.00 & 5.07 & 0.00 & 0.00 & 0.03 & 0.00 & 1.73 & 0.12 & 0.00 \\
\hline$\left|\tilde{t}\left(r_{1}\right)\right|$ & 0.02 & 0.00 & 5.07 & 0.00 & 0.00 & 0.03 & 0.00 & 1.73 & 0.12 & 0.00 \\
\hline$\left|t\left(\hat{\rho}_{k}\right)\right|$ & 0.02 & 0.00 & 4.86 & 0.00 & 0.00 & 0.02 & 0.00 & 1.60 & 0.12 & 0.00 \\
\hline$\left|\bar{t}\left(\hat{\rho}_{k}\right)\right|$ & 0.03 & 0.00 & 5.10 & 0.00 & 0.00 & 0.02 & 0.01 & 1.74 & 0.18 & 0.00 \\
\hline & \multicolumn{10}{|c|}{ Tests based on model-specific size correction } \\
\hline$\left|t\left(r_{1}\right)\right|$ & 4.86 & 5.01 & 5.07 & 5.10 & 5.30 & 5.17 & 5.15 & 5.24 & 4.94 & 5.01 \\
\hline$\left|\tilde{t}\left(r_{1}\right)\right|$ & 4.86 & 5.01 & 5.07 & 5.10 & 5.30 & 5.17 & 5.15 & 5.24 & 4.94 & 5.01 \\
\hline$\left|t\left(\hat{\rho}_{k}\right)\right|$ & 4.80 & 4.73 & 4.86 & 4.90 & 5.17 & 5.15 & 5.23 & 5.35 & 5.22 & 4.87 \\
\hline$\left|\bar{t}\left(\hat{\rho}_{k}\right)\right|$ & 4.60 & 4.93 & 5.10 & 5.29 & 4.77 & 5.11 & 5.04 & 5.22 & 5.10 & 5.28 \\
\hline Sample size: $n=60$ & \multicolumn{10}{|c|}{ Asymptotic tests and bounds } \\
\hline$\left|t\left(r_{1}\right)\right|$ & 4.20 & 2.83 & 50.90 & 65.69 & 66.21 & 2.88 & 2.92 & 30.90 & 30.08 & 48.90 \\
\hline$\left|\tilde{t}\left(r_{1}\right)\right|$ & 4.89 & 3.22 & 51.92 & 66.58 & 67.12 & 3.07 & 3.09 & 31.61 & 30.75 & 49.77 \\
\hline$\left|t\left(\hat{\rho}_{k}\right)\right|$ & 4.24 & 2.81 & 49.98 & 65.08 & 65.57 & 2.84 & 2.88 & 30.30 & 29.55 & 48.09 \\
\hline$\left|\bar{t}\left(\hat{\rho}_{k}\right)\right|$ & 4.77 & 3.05 & 51.27 & 65.82 & 66.49 & 2.95 & 3.13 & 31.19 & 30.39 & 49.12 \\
\hline$E_{11}$ & 0.72 & 0.91 & 2.31 & 0.62 & 0.00 & 1.15 & 1.08 & 2.95 & 1.45 & 0.00 \\
\hline \multirow[t]{2}{*}{ Best bound } & 1.01 & 1.21 & 2.31 & 0.62 & 0.00 & 1.24 & 1.10 & 2.95 & 1.45 & 0.00 \\
\hline & \multicolumn{10}{|c|}{ Tests based on Imhof critical values } \\
\hline$\left|t\left(r_{1}\right)\right|$ & 4.99 & 3.29 & 52.05 & 66.69 & 67.23 & 3.12 & 3.12 & 31.75 & 30.90 & 49.87 \\
\hline$\left|\tilde{t}\left(r_{1}\right)\right|$ & 5.20 & 3.48 & 52.33 & 66.96 & 67.59 & 3.21 & 3.19 & 31.93 & 31.12 & 50.17 \\
\hline$\left|t\left(\hat{\rho}_{k}\right)\right|$ & 5.12 & 3.34 & 51.29 & 66.19 & 66.70 & 3.09 & 3.09 & 31.25 & 30.51 & 49.13 \\
\hline \multirow{2}{*}{$\left|\bar{t}\left(\hat{\rho}_{k}\right)\right|$} & 4.95 & 3.16 & 51.85 & 66.29 & 66.91 & 3.07 & 3.20 & 31.57 & 30.81 & 49.55 \\
\hline & \multicolumn{10}{|c|}{ Tests based on global size correction } \\
\hline$\left|t\left(r_{1}\right)\right|$ & 0.00 & 0.00 & 5.09 & 0.00 & 0.00 & 0.00 & 0.00 & 1.86 & 0.08 & 0.00 \\
\hline$\left|\tilde{t}\left(r_{1}\right)\right|$ & 0.00 & 0.00 & 5.09 & 0.00 & 0.00 & 0.00 & 0.00 & 1.86 & 0.08 & 0.00 \\
\hline$\left|t\left(\hat{\rho}_{k}\right)\right|$ & 0.00 & 0.00 & 5.20 & 0.00 & 0.00 & 0.00 & 0.00 & 1.88 & 0.08 & 0.00 \\
\hline \multirow[t]{2}{*}{$\left|\bar{t}\left(\hat{\rho}_{k}\right)\right|$} & 0.00 & 0.00 & 5.03 & 0.00 & 0.00 & 0.00 & 0.00 & 1.88 & 0.07 & 0.00 \\
\hline & \multicolumn{10}{|c|}{ Tests based on model-specific size correction } \\
\hline$\left|t\left(r_{1}\right)\right|$ & 4.72 & 5.26 & 5.07 & 5.19 & 4.83 & 5.12 & 4.99 & 5.42 & 4.83 & 4.65 \\
\hline$\left|\tilde{t}\left(r_{1}\right)\right|$ & 4.72 & 5.26 & 5.07 & 5.19 & 4.83 & 5.12 & 4.99 & 5.42 & 4.83 & 4.65 \\
\hline$\left|t\left(\hat{\rho}_{k}\right)\right|$ & 4.59 & 5.09 & 5.11 & 5.34 & 4.96 & 5.03 & 4.85 & 5.35 & 4.85 & 5.03 \\
\hline$\left|\bar{t}\left(\hat{\rho}_{k}\right)\right|$ & 4.67 & 5.28 & 5.07 & 5.16 & 4.95 & 5.07 & 4.98 & 5.35 & 4.61 & 4.93 \\
\hline
\end{tabular}


Table 3. Empirical powers of serial dependence tests at level $\alpha=0.05$

$$
X_{t}=0.2 X_{t-1}+u_{t}
$$

\begin{tabular}{|c|c|c|c|c|c|c|c|c|c|c|}
\hline \multirow{2}{*}{$\begin{array}{c}\text { Sample size: } n=30 \\
\text { Error distribution }\left(v_{t}\right)\end{array}$} & \multicolumn{10}{|c|}{ Asymptotic tests and bounds } \\
\hline & \multicolumn{5}{|c|}{$\mathrm{N}(0,1)$} & \multicolumn{5}{|c|}{ Cauchy } \\
\hline Test $\backslash$ Model & M1 & M2 & M5 & M7 & M8 & M1 & M2 & M5 & M7 & M8 \\
\hline$E_{11}$ & 4.81 & 7.15 & 3.51 & 19.51 & 48.06 & 13.37 & 14.59 & 7.29 & 19.78 & 44.49 \\
\hline \multirow[t]{2}{*}{ Best bound } & 5.66 & 7.54 & 3.51 & 19.52 & 48.06 & 13.59 & 14.72 & 7.29 & 19.78 & 44.49 \\
\hline & \multicolumn{10}{|c|}{ Tests based on global size correction } \\
\hline$\left|t\left(r_{1}\right)\right|$ & 0.22 & 0.02 & 7.77 & 0.19 & 0.00 & 0.08 & 0.11 & 2.98 & 0.66 & 0.00 \\
\hline$\left|\tilde{t}\left(r_{1}\right)\right|$ & 0.22 & 0.02 & 7.77 & 0.19 & 0.00 & 0.08 & 0.11 & 2.98 & 0.66 & 0.00 \\
\hline$\left|t\left(\hat{\rho}_{k}\right)\right|$ & 0.12 & 0.02 & 6.61 & 0.03 & 0.00 & 0.03 & 0.08 & 2.36 & 0.25 & 0.00 \\
\hline \multirow[t]{2}{*}{$\left|\bar{t}\left(\hat{\rho}_{k}\right)\right|$} & 0.37 & 0.05 & 8.57 & 0.60 & 0.00 & 0.10 & 0.13 & 3.48 & 0.83 & 0.00 \\
\hline & \multicolumn{10}{|c|}{ Tests based on model-specific size correction } \\
\hline$\left|t\left(r_{1}\right)\right|$ & 18.71 & 23.05 & 7.77 & 24.77 & 25.27 & 16.03 & 16.16 & 8.59 & 14.08 & 16.75 \\
\hline$\left|\tilde{t}\left(r_{1}\right)\right|$ & 18.71 & 23.05 & 7.77 & 24.77 & 25.27 & 16.03 & 16.16 & 8.59 & 14.08 & 16.75 \\
\hline$\left|t\left(\hat{\rho}_{k}\right)\right|$ & 12.36 & 13.78 & 6.61 & 20.38 & 20.32 & 10.95 & 10.72 & 7.37 & 11.67 & 13.63 \\
\hline$\left|\bar{t}\left(\hat{\rho}_{k}\right)\right|$ & 17.73 & 21.54 & 8.57 & 24.93 & 25.26 & 15.71 & 15.91 & 8.99 & 14.90 & 17.42 \\
\hline Sample size: $n=60$ & \multicolumn{10}{|c|}{ Asymptotic tests and bounds } \\
\hline$E_{1}$ & 11.54 & 13.92 & 3.54 & 21.85 & 49.02 & 26.04 & 25.75 & 7.40 & 25.78 & 44.41 \\
\hline \multirow[t]{2}{*}{ Best bound } & 13.71 & 15.26 & 3.55 & 21.85 & 49.02 & 26.32 & 26.09 & 7.40 & 25.79 & 44.41 \\
\hline & \multicolumn{10}{|c|}{ Tests based on global size correction } \\
\hline$\left|t\left(r_{1}\right)\right|$ & 0.00 & 0.00 & 8.00 & 0.11 & 0.00 & 0.01 & 0.00 & 3.16 & 0.33 & 0.00 \\
\hline$\left|\tilde{t}\left(r_{1}\right)\right|$ & 0.00 & 0.00 & 8.00 & 0.11 & 0.00 & 0.0 & 0.00 & 3.16 & 0.33 & 0.00 \\
\hline$\left|t\left(\hat{\rho}_{k}\right)\right|$ & 0.00 & 0.00 & 7.54 & 0.00 & 0.00 & 0.00 & 0.00 & 2.88 & 0.25 & 0.00 \\
\hline$\left|\bar{t}\left(\hat{\rho}_{k}\right)\right|$ & 0.01 & 0.00 & 8.33 & 0.20 & 0.00 & 0.02 & 0.00 & 3.37 & 0.36 & 0.00 \\
\hline & \multicolumn{10}{|c|}{ Tests based on model-specific size correction } \\
\hline$\left|t\left(r_{1}\right)\right|$ & 33.22 & 41.38 & 8.00 & 24.79 & 25.42 & 43.25 & 42.27 & 8.66 & 12.34 & 17.18 \\
\hline$\left|\tilde{t}\left(r_{1}\right)\right|$ & 33.22 & 41.38 & 8.00 & 24.79 & 25.42 & 43.25 & 42.27 & 8.66 & 12.34 & 17.18 \\
\hline$\left|t\left(\hat{\rho}_{k}\right)\right|$ & 27.21 & 33.31 & 7.54 & 22.97 & 23.38 & 29.29 & 27.99 & 8.14 & 11.11 & 16.55 \\
\hline$\left|\bar{t}\left(\hat{\rho}_{k}\right)\right|$ & 32.26 & 39.92 & 8.33 & 25.20 & 25.75 & 41.73 & 41.69 & 9.00 & 12.50 & 18.09 \\
\hline
\end{tabular}


Table 4. Empirical powers of serial dependence tests at level $\alpha=0.05$

$$
X_{t}=0.9 X_{t-1}+u_{t}
$$

\begin{tabular}{|c|c|c|c|c|c|c|c|c|c|c|}
\hline Sample size: $n=30$ & \multicolumn{10}{|c|}{ Asymptotic tests and bounds } \\
\hline Error distribution $\left(v_{t}\right)$ & \multicolumn{5}{|c|}{$\mathrm{N}(0,1)$} & \multicolumn{5}{|c|}{ Cauchy } \\
\hline Test $\backslash$ Model & M1 & M2 & M5 & M7 & M8 & M1 & M2 & M5 & M7 & M8 \\
\hline$E_{11}$ & 97.94 & 97.95 & 19.45 & 83.83 & 84.70 & 94.39 & 94.67 & 35.89 & 90.42 & 89.65 \\
\hline \multirow[t]{2}{*}{ Best bound } & 98.20 & 98.18 & 19.45 & 84.21 & 85.06 & 94.55 & 94.92 & 35.95 & 90.81 & 89.97 \\
\hline & \multicolumn{10}{|c|}{ Tests based on global size correction } \\
\hline$\left|\underset{\sim}{t}\left(r_{1}\right)\right|$ & 92.69 & 97.09 & 40.57 & 79.63 & 79.43 & 93.46 & 94.40 & 56.06 & 88.42 & 86.01 \\
\hline$\left|\tilde{t}\left(r_{1}\right)\right|$ & 92.69 & 97.09 & 40.57 & 79.63 & 79.43 & 93.46 & 94.40 & 56.06 & 88.42 & 86.01 \\
\hline$\left|t\left(\hat{\rho}_{k}\right)\right|$ & 80.15 & 90.72 & 40.76 & 71.37 & 71.49 & 86.36 & 87.83 & 54.99 & 81.97 & 80.07 \\
\hline \multirow[t]{2}{*}{$\left|\bar{t}\left(\hat{\rho}_{k}\right)\right|$} & 85.21 & 93.59 & 47.30 & 73.70 & 73.76 & 89.35 & 90.57 & 61.06 & 84.20 & 81.78 \\
\hline & \multicolumn{10}{|c|}{ Tests based on model-specific size correction } \\
\hline$\left|t\left(r_{1}\right)\right|$ & 99.65 & 99.95 & 40.57 & 84.29 & 83.85 & 98.99 & 99.09 & 71.44 & 92.65 & 88.98 \\
\hline$\left|\tilde{t}\left(r_{1}\right)\right|$ & 99.65 & 99.95 & 40.57 & 84.29 & 83.85 & 98.99 & 99.09 & 71.44 & 92.65 & 88.98 \\
\hline$\left|t\left(\hat{\rho}_{k}\right)\right|$ & 98.52 & 99.71 & 40.76 & 77.68 & 77.46 & 98.28 & 98.41 & 71.21 & 88.70 & 84.87 \\
\hline$\left|\bar{t}\left(\hat{\rho}_{k}\right)\right|$ & 99.06 & 99.84 & 47.30 & 79.27 & 79.06 & 98.59 & 98.64 & 77.24 & 89.86 & 85.98 \\
\hline Sample size: $n=60$ & \multicolumn{10}{|c|}{ Asymptotic tests and bounds } \\
\hline$E_{11}$ & 100 & 100 & 18.45 & 86.29 & 85.85 & 99.09 & 99.15 & 35.00 & 96.03 & 90.32 \\
\hline \multirow[t]{2}{*}{ Best bound } & 100 & 100 & 18.45 & 86.55 & 86.23 & 99.11 & 99.18 & 35.10 & 96.28 & 90.52 \\
\hline & \multicolumn{10}{|c|}{ Tests based on global size correction } \\
\hline$\left|t\left(r_{1}\right)\right|$ & 99.11 & 99.55 & 39.31 & 80.56 & 80.27 & 97.93 & 98.15 & 55.43 & 92.95 & 86.61 \\
\hline$\left|\tilde{t}\left(r_{1}\right)\right|$ & 99.11 & 99.55 & 39.31 & 80.56 & 80.27 & 97.93 & 98.15 & 55.43 & 92.95 & 86.61 \\
\hline$\left|t\left(\hat{\rho}_{k}\right)\right|$ & 97.54 & 98.57 & 40.00 & 76.78 & 75.75 & 97.02 & 97.44 & 55.51 & 90.50 & 83.31 \\
\hline$\left|\bar{t}\left(\hat{\rho}_{k}\right)\right|$ & 98.11 & 98.92 & 42.82 & 77.46 & 76.66 & 97.43 & 97.68 & 58.09 & 91.07 & 83.98 \\
\hline & \multicolumn{10}{|c|}{ Tests based on model-specific size correction } \\
\hline$\left|\underset{\sim}{t}\left(r_{1}\right)\right|$ & 100 & 100 & 39.31 & 84.95 & 84.35 & 99.68 & 99.62 & 70.70 & 96.31 & 89.96 \\
\hline$\left|\tilde{t}\left(r_{1}\right)\right|$ & 100 & 100 & 39.31 & 84.95 & 84.35 & 99.68 & 99.62 & 70.70 & 96.31 & 89.96 \\
\hline$\left|t\left(\hat{\rho}_{k}\right)\right|$ & 100 & 100 & 40.00 & 81.52 & 81.11 & 99.58 & 99.57 & 70.87 & 94.98 & 87.73 \\
\hline$\left|\bar{t}\left(\hat{\rho}_{k}\right)\right|$ & 100 & 100 & 42.82 & 82.35 & 81.69 & 99.60 & 99.59 & 74.26 & 95.35 & 88.13 \\
\hline
\end{tabular}


ensuring that the probability of rejecting the null hypothesis of independence is not larger than the level) in other cases covered by the null hypothesis of independence (e.g., with heteroskedasticity); for further discussion of the Imhof algorithm, see Imhof (1961), Koerts and Abrahamse (1969) and Dufour and King (1991). The third method provides a theoretical benchmark that cannot be achieved in practice, because the heteroskedasticity pattern is not specified by the null hypothesis of independence. The fourth method is the one closest to what one would like to do for a distributionfree test that is robust to non-normality and heteroskedasticity of unknown form, based on these statistics. It is not clear, however, that the (marginal) distributions of these statistics can be bounded in a useful way under the (very wide) null hypothesis considered by the conditional bounds we propose [for further of discussion of this point, see Pratt and Gibbons (1981, chapter 4), Dufour and Hallin (1991, section 1), Dufour (2003, section 4.2)]. We do not have a way of producing provably valid critical values for these tests. So the "size-corrected" critical values used for the unconditional tests remain too "small" and the powers presented overestimate the true power of these procedures for the nonparametric null hypothesis studied.

All tests are performed at the 0.05 nominal level. Sample sizes $n=30,60$ were considered. Results on empirical frequencies of type I errors (empirical level) appear in Table 2, while powers for $\varphi=0.2,0.9$ appear in tables $3-4{ }^{1}$ Size and power frequencies were evaluated using 10000 replications. Critical values for the "size-corrected" tests were obtained out of a preliminary simulation involving 100000 replications. Most calculations were performed with Fortran 90 programs (Sun Workshop Compiler Fortran 90 4.2) running on a Unix server. Critical values based on the Imhof algorithm were obtained using the SHAZAM version 9) computer program [Whistler, White, Wong and Bates (2001)].

We see from these results that the bounds constitute the only method that allows one to control the level of the test for all the patterns considered, in the sense that the probability of type I error is never larger than the nominal level 0.05 of the test. By contrast, the probability of type I of error can get as large as 0.54 for $n=30$ and 0.65 for $n=60$ in the limited number cases considered in this experiment, so the size of the tests considered is at least as large as these numbers, even though the nominal size is $0.05 .^{2}$ In particular, tests based on exact critical values designed for i.i.d. Gaussian observations behave very poorly in such circumstances.

Once standard tests are corrected for size, the bounds can lead to substantial power gains. This holds despite the fact that our "size corrections" are incomplete, so the powers of the tests that are not based on bounds are overestimated. The adjustments required to correct the size of these procedures are simply too "large" to yield useful tests of the nonparametric hypothesis considered. This shows clearly that the distribution-free bounds presented in this paper can at least provide a useful check on the reliability of serial dependence tests that are not provably distribution-free.

We also observed that the tightest exponential $E_{11}=B_{1}\left(y_{1},|X|\right)$ yields the best results in terms of power (for a level of 0.05 ), with a performance that is very close to the one provided by the minimum value over all the bounds (which may be supplied by a different bound, depending on the

\footnotetext{
${ }^{1}$ The complete results of our experiment are reported in Appendix C.

${ }^{2}$ Under a sufficiently important heteroskedasticity, it is not clear that traditional test statistics have the usual asymptotic normal distribution, so there is no presumption that standard asymptotic theory will work well here or exhibit convergence. This can be contrasted with the fact that the conditional distribution-free tests proposed here are provably exact under in same crcumstances.
} 
sample).

\section{Application to commercial paper rate}

In this section, we illustrate how the bounds derived above can be used by applying them to U.S. data on interest rates. We will study the autocorrelation structure of the first and second differences of the logarithm of the commercial paper rate [denoted by $\ln \left(r_{t}\right)$ ] from 1951 to 1983 (quarterly, 132 observations). The source of the data is Balke and Gordon (1986, pp. 789-808).

For these two series, we report in Tables 5 and 6 the usual centered version of traditional autocorrelations $\hat{\rho}_{k}$ [defined in (5.7)] and the uncentered autocorrelations $r_{k}$ [in (2.1)], for $k=1, \ldots, 20$. Since both series have means very close to zero, there is very little difference between the two sets of autocorrelation coefficients (see also the $t$-statistics reported in the tables). Even though we are mostly interested by the minimal upper bound on the $p$-value for testing independence, we also report the individual bounds for the sake of comparison (but one would not normally report all this information). The bounds reported are for two-sided tests, i.e., we compute bounds on $\mathrm{P}\left[\left|r_{k}\right| \geq y|| X \mid\right]=2 \mathrm{P}\left[r_{k} \geq|y||| X \mid\right]$ at $y=\hat{r}_{k}$ (observed value of $r_{k}$ ). The upper bounds on $\mathrm{P}\left[r_{k} \geq|y||| X \mid\right]$ computed are based on the four exponential bounds $E_{1 k} \leq E_{2 k} \leq E_{3 k} \leq E_{4 k}$ from (2.3), the improved Eaton-Pinelis-type bounds $B_{E P}^{*}$ and $B_{E P}$ from (3.1), Chebyshev bounds based on the exact conditional even moments of $r_{k}$, binomial moments and normal moments as given in (3.4) - (3.5), and the Berry-Esséen-Zolotarev type bound $B E_{U}$ given by (4.2). The Chebyshev bound $(C)$ based on the exact moments of $r_{k}$ is the minimal value yielded by the six first even moments $(p=2,4, \ldots, 12)$, the one based on the binomial moments is the best over the first 15 even moments $(p=2,4, \ldots, 30)$, while the normal moment bound is based on (3.5). We also report the Berry-Esséen lower bound obtained from (4.2). All the upper bounds we consider (except $B_{E P}^{*}$ and $B_{E P}$ ) can take values larger than 1.0 : since a probability cannot be greater than 1.0, any one of these bounds can be improved by taking the minimum given by the bound and 1.0. Consequently, when an upper bound exceeds one, we report 1.0 in the table. Similarly, when the lower bound is less than zero, we report 0.0 in the table.

From the results in Table 5, we see that the series $X_{t}=(1-B) \ln \left(r_{t}\right)=\ln \left(r_{t}\right)-\ln \left(r_{t-1}\right)$ exhibits four autocorrelations $r_{k}$ (at lags $\left.k=1,2,6,7\right)$ whose absolute values exceed two asymptotic standard errors $\left(\left|r_{k}\right| \geq 2 / \sqrt{n}=2 / \sqrt{131}=0.175\right)$. Among these, three $(k=1,6,7)$ are clearly significant at level 0.05 under the assumption that $X_{1}, \ldots, X_{n}$ have distributions symmetric about zero. It is also of interest to note that the autocorrelations at lags $k=3,5,8,9,12,13,14,18$ are clearly not significant at level 0.05 . Depending on cases, the best upper bound is obtained by using either a Chebyshev $(C)$, Eaton-type $\left(B_{E P}^{*}\right)$ or Berry-Esséen bound.

The autocorrelations for the second differences $X_{t}=(1-B)^{2} \ln \left(r_{t}\right)$ in Table 6 exhibit only one autocorrelation (at $k=2$ ) whose absolute value is greater than two asymptotic standard errors $\left(\left|r_{k}\right| \geq 2 / \sqrt{n}=2 / \sqrt{130}=0.175\right)$. The nonparametric upper bound on the $p$-value for $\left|r_{2}\right|$ indicates that this is significant even for a level as low as 0.00002; the best upper bound is given here by the exponential bound $E_{1}$. In this case, all the upper bounds (except the Berry-Esséen one) indicate that this is significant at level 0.005. The Berry-Esséen lower bound indicates that the autocorrelations at lags $k=5,12,13$ are clearly not significant at level 0.05 . Overall the second differences 


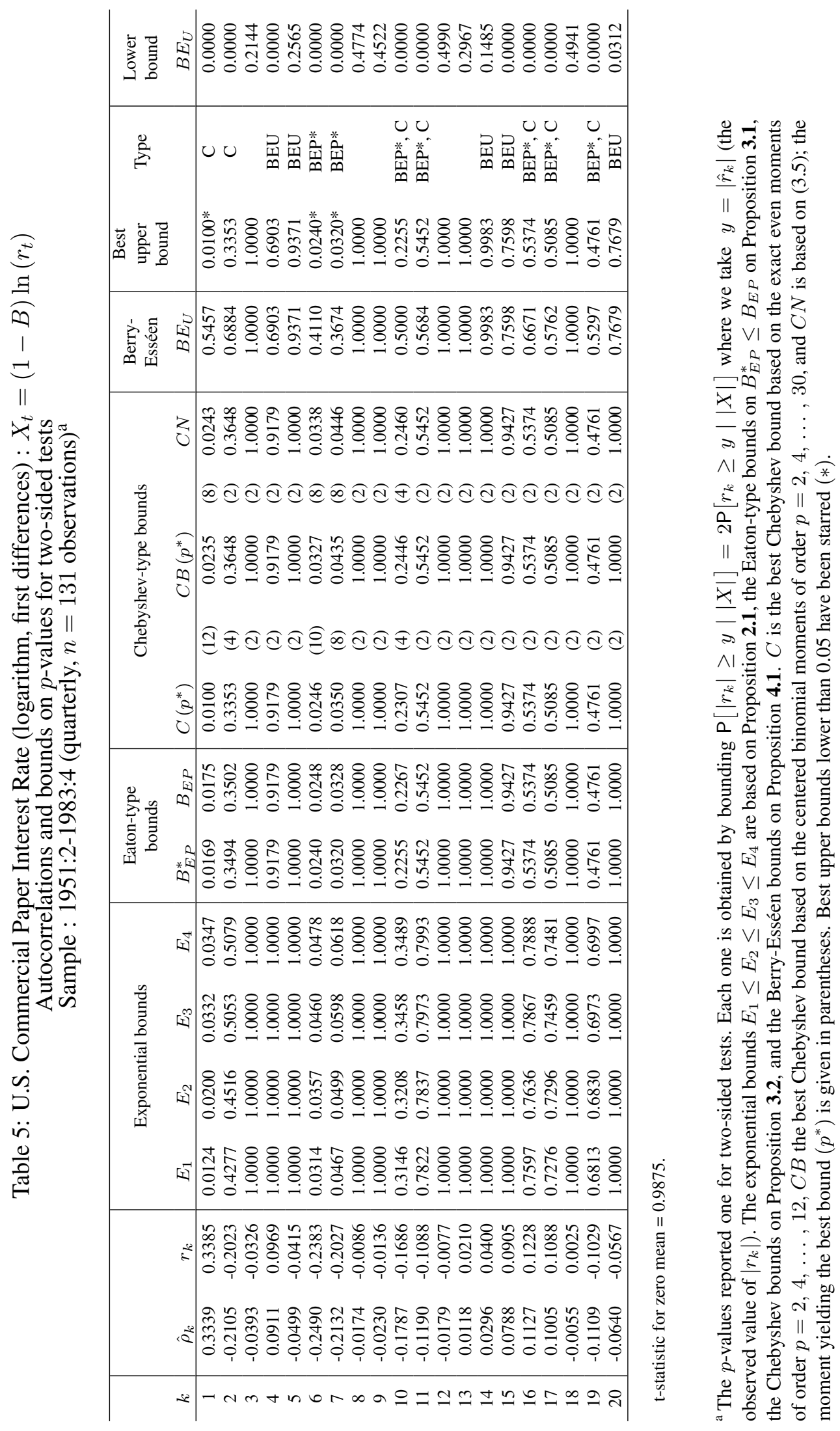




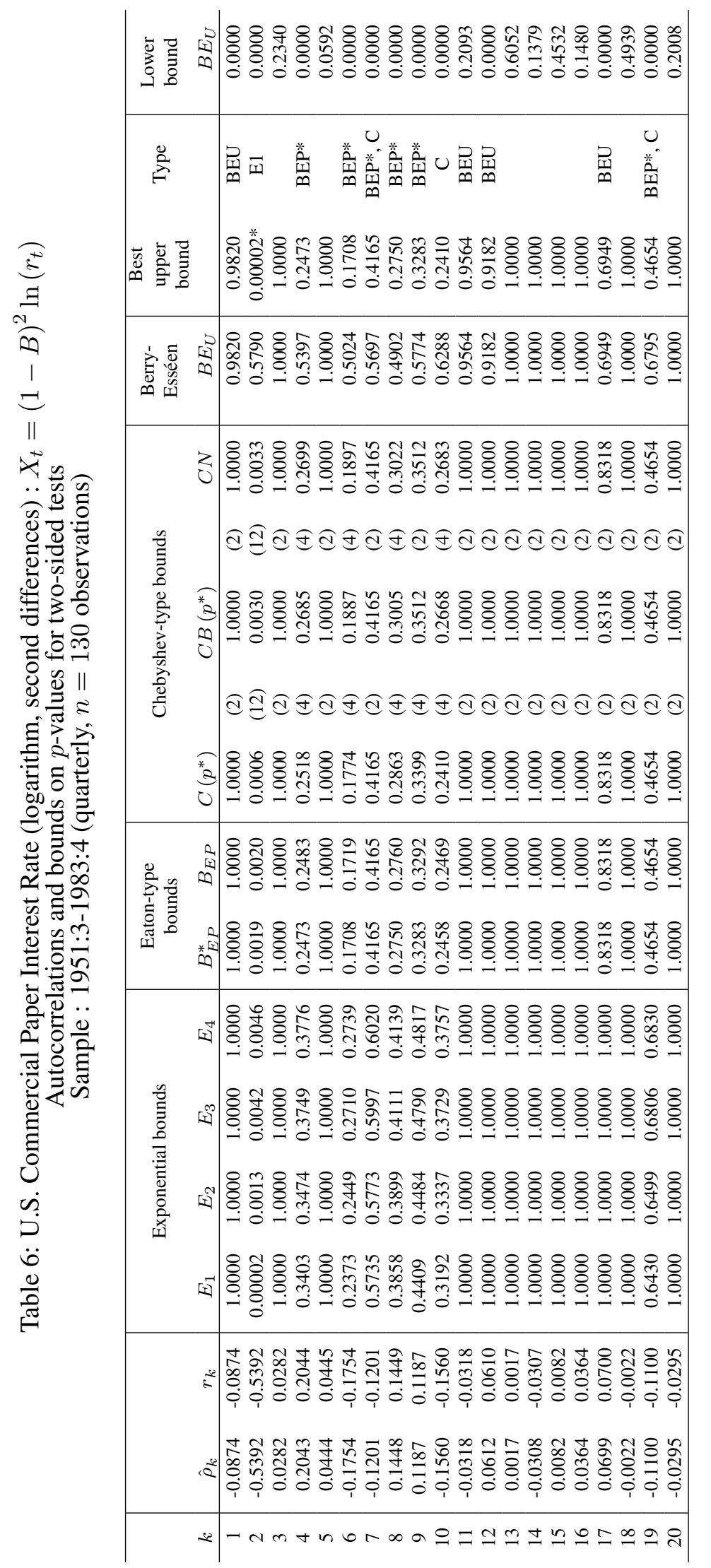

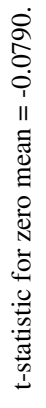


of $\ln \left(r_{t}\right)$ seem to have a simpler autocorrelation structure than the first differences $(1-B) \ln \left(r_{t}\right)$.

\section{Conclusion}

In this paper, we suggested several ways of bounding the distribution of serial correlation coefficients, under a nonparametric null hypothesis of serial independence, allowing for both discrete and continuous distributions as well as general heterogeneity of unknown form. As required in the case of a sufficiently general heteroskedasticity, the proposed technique is based on the conditional distribution of the autocorrelations given the absolute values of the observations, which is then bounded by considering the distribution of the signs. The bounds proposed are valid for any sample size and do not rely on asymptotic approximations. In order to do that we assumed that the observations have symmetric (non-identical) distributions with respect to known medians.

These are, of course, real restrictions. But minimal distributional assumptions are needed to get testable hypotheses. The symmetry assumption is quite common in econometrics and statistics and holds for many important distributional families [e.g., Gaussian distributions, Cauchy distributions, a wide class a stable laws, etc.]. Relaxing it will require the introduction of alternative assumptions, such as i.i.d. observations (which precludes heteroskedasticity); see Dufour and Roy (1985) and Hallin and Puri (1992). Of course, which set of restrictions is most appropriate will depend on the context.

The assumption that the observations have known medians can be relaxed more easily. For example, if we assume that the observations have the same median, it is possible to obtain an exact confidence interval for this unknown median (which plays the role of a nuisance parameter), for example by inverting a sign test or the nonparametric $t$ test described in Dufour and Hallin (1991). One can then test test serial independence by using use a two-stage confidence procedure similar to the ones proposed in Dufour (1990), Campbell and Dufour (1997) and Dufour and Kiviet (1998) in other contexts. Designing such a procedure, or alternative ones that would deal such nuisance parameters, goes beyond the scope of the present article and will be considered in a subsequent paper. 


\section{A. Appendix: Proofs}

Proof of Proposition 2.1 Let $Z_{k t}=X_{t} X_{t+k}, t=1, \ldots, n-k$, and let $\operatorname{sgn}(x)$ be the sign function: $\operatorname{sgn}(x)=-1$ if $x<0,0$ if $x=0$, and 1 if $x>0$.Then we can write

$$
r_{k}=D_{k}(|X|) \sum_{t=1}^{n-k} w_{k t} S_{k t}=D_{k}(|X|) R_{k}
$$

where $S_{k t}=\operatorname{sgn}\left(Z_{k t}\right), t=1, \ldots, n-k, R_{k}=\sum_{t=1}^{n-k} w_{k t} S_{k t}$, and $\sum w_{k t}^{2}=1$. When $Z_{k 1}=\cdots=Z_{k, n-k}=0$, we have $r_{k}=D_{k}(|X|)=0$, so that $\mathrm{P}\left[r_{k} \geq y|| X \mid\right]=0$, a.s., and the result holds trivially. We now suppose that $Z_{k t} \neq 0$ for at least one $t$. Let $A_{k}(|X|)=\left\{t:\left|X_{t}\right| \neq 0,1 \leq t \leq n-k\right\}$ and $B_{k}(|X|)=\left\{t:\left|X_{t} X_{t+k}\right| \neq 0,1 \leq t \leq n-k\right\}$. Clearly, $t \in B_{k}(|X|)$ if and only if $t \in A_{k}(|X|)$ and $t+k \in A_{k}(|X|)$, and

$$
R_{k}=\sum_{t=1}^{n-k} w_{k t} S_{k t}=\sum_{t \in B_{k}(|X|)} w_{k t} S_{k t} .
$$

By the independence of $X_{1}, \ldots, X_{n}$ and by the symmetry assumption, the variables in the set $\left\{\operatorname{sgn}\left(X_{t}\right): t \in A_{k}(|X|)\right\}$ are independent conditional on $|X|$, with $\mathrm{P}\left[\operatorname{sgn}\left(X_{t}\right)=-1|| X \mid\right]=$ $\mathrm{P}\left[\operatorname{sgn}\left(X_{t}\right)=1|| X \mid\right]=0.5$. Further, since $\operatorname{sgn}\left(Z_{k t}\right)=\operatorname{sgn}\left(X_{t}\right) \operatorname{sgn}\left(X_{t+k}\right)$, it is easy to see that the variables in the set $\left\{S_{k t}: t \in B_{k}(|X|)\right\}$ are independent conditional on $|X|$ with

$$
\mathrm{P}\left[S_{k t}=-1|| X \mid\right]=\mathrm{P}\left[S_{k t}=1|| X \mid\right]=0.5 ;
$$

see Dufour (1981). It is clear from (A.1) - (A.3) that the conditional distribution of $r_{k}$ given $|X|$ is symmetric about zero. Further, using Markov's inequality and observing that $\cosh \left(w_{k t} z\right)=$ $\cosh (0)=1$ for $t \notin B_{k}(|X|)$, we have:

$$
\mathrm{P}\left[R_{k} \geq y|| X \mid\right] \leq \mathrm{E}\left[\exp \left(z R_{k}|| X \mid\right)\right] / \exp (z y)=\prod_{t=1}^{n-k} \cosh \left(w_{k t} z\right) / \exp (z y)
$$

for all $z \geq 0$ and for all $y$. Consequently, for all $y>0$,

$$
\begin{aligned}
\mathrm{P}\left[R_{k} \geq y|| X \mid\right] & \leq \inf _{z \geq 0}\left\{\exp (-z y) \prod_{t=1}^{n-k} \cosh \left(w_{k t} z\right)\right\} \leq \exp \left(-y^{2}\right) \prod_{t=1}^{n-k} \cosh \left(w_{k t} y\right) \\
& =\exp \left(-y^{2}\right) \prod_{t \in B_{k}(|X|)} \cosh \left(w_{k t} y\right) \leq \exp \left(-y^{2}\right)\left\{\cosh \left(y / \sqrt{n_{k}^{*}}\right)\right\}^{n_{k}^{*}} \\
& <\exp \left(-y^{2}\right)\left\{\exp \left[\left(y / \sqrt{n_{k}^{*}}\right)^{2} / 2\right]\right\}^{n_{k}^{*}}=\exp \left(-y^{2} / 2\right)
\end{aligned}
$$

where the second inequality is obtained by taking $z=y$ in (A.4), the third one follows from Corollary 1 and Example 2 of Eaton (1970), and the last one is obtained by noting that cosh $(x)<$ 
$\exp \left(x^{2} / 2\right)$ for $x>0$ [Edelman (1986)]. Inequality (2.3) follows from (A.4) on observing that $r_{k}=D_{k}(|X|) R_{k}$.

Proof of Proposition 3.1 When $X_{t} X_{t+k}=0$, for $t=1, \ldots, n-k$, we have $r_{k}=0$ and (3.1) clearly holds. When $X_{t} X_{t+k} \neq 0$ for some $t$, the result follows from (A.1) to (A.3), and then by applying Proposition 1 from Dufour and Hallin (1993) to $R_{k}$ in (A.2).

Proof of Proposition 3.2 When $X_{t} X_{t+k}=0$, for $t=1, \ldots, n-k$, we have $r_{k}=0$, and (3.4)-(3.5) clearly hold. Otherwise, the result follows from (A.1) to (A.3), and Proposition 2 in Dufour and Hallin (1992).

Proof of Proposition 4.1 The result is immediate from (A.1) to (A.3) and Proposition 3 from Dufour and Hallin (1992).

\section{B. Appendix: Conditional moments of the autocorrelations}

The conditional moments $\mathrm{E}\left(r_{k}^{p}|| X \mid\right)$ in (3.4) can be computed by noting that

$$
\mathrm{E}\left(r_{k}^{p}|| X \mid\right)=D_{k}(|X|)^{p} \mathrm{E}\left(R_{k}^{p}|| X \mid\right)
$$

where, provided $D_{k}(|X|) \neq 0$ (otherwise, $r_{k}=0$ ), $\mathrm{E}\left(R_{k}^{2}|| X \mid\right)=1$ and, for $p=4,6, \ldots, 12$, $\mathrm{E}\left(R_{k}^{p}|| X \mid\right)$ is given by the following formulae: setting $W_{k p}=\sum_{t=1}^{n-k} w_{k t}^{p}$,

$$
\begin{aligned}
\mathrm{E}\left(R_{k}^{4}|| X \mid\right)= & 3-2 W_{k 4} \\
\mathrm{E}\left(R_{k}^{6}|| X \mid\right)= & 15-30 W_{k 4}+16 W_{k 6} \\
\mathrm{E}\left(R_{k}^{8}|| X \mid\right)= & 105-420 W_{k 4}+140 W_{k 4}^{2}+448 W_{k 6}-272 W_{k 8} \\
\mathrm{E}\left(R_{k}^{10}|| X \mid\right)= & 945-6300 W_{k 4}+6300 W_{k 4}^{2}+10080 W_{k 6}-6720 W_{k 6} W_{k 4} \\
& -12240 W_{k 8}+7936 W_{k, 10}, \\
\mathrm{E}\left(R_{k}^{12}|| X \mid\right)= & 10395-103950 W_{k 4}+207900 W_{k 4}^{2}-46200 W_{k 4}^{3}+221760 W_{k 6} \\
& -443520 W_{k 6} W_{k 4}+118272 W_{k 6}^{2}-403920 W_{k 8} \\
& +269280 W_{k 8} W_{k 4}+523776 W_{k, 10}-353792 W_{k, 12} .
\end{aligned}
$$

\section{Appendix: Additional simulation results}

In this appendix, we report more complete results on our simulation experiment. In particular, we give results for three sample sizes $(n=30,60,100)$ and more values of the autoregressive coefficient $(\varphi=0.2,0.3,0.4,0.5,0.6,0.8,0.9)$. The empirical levels are reported in tables C.1 C.3, the estimated critical values for correcting size in the the power study in table C.4, while the results on power appear in tables C.5 - C.18. 
Table C.1. Empirical levels of serial dependence tests at nominal level $\alpha=0.05 ; n=30$

\begin{tabular}{|c|c|c|c|c|c|c|c|c|}
\hline$v_{t} \sim \mathrm{N}(0,1)$ & \multicolumn{8}{|c|}{ Asymptotic tests and bounds } \\
\hline Heteroskedasticity type & M1 & M2 & M3 & M4 & M5 & M6 & M7 & M8 \\
\hline$\left|t\left(r_{1}\right)\right|$ & 3.90 & 1.67 & 0.27 & 16.75 & 33.60 & 29.91 & 49.46 & 51.32 \\
\hline$\left|\tilde{t}\left(r_{1}\right)\right|$ & 4.96 & 2.20 & 0.36 & 19.21 & 36.17 & 33.50 & 52.26 & 54.42 \\
\hline$\left|t\left(\hat{\rho}_{k}\right)\right|$ & 4.22 & 1.91 & 0.27 & 16.49 & 31.78 & 27.81 & 47.29 & 49.11 \\
\hline$\left|\bar{t}\left(\hat{\rho}_{k}\right)\right|$ & 4.65 & 2.17 & 0.30 & 18.13 & 34.45 & 31.06 & 49.90 & 51.95 \\
\hline$E_{11}$ & 0.95 & 0.87 & 0.87 & 1.27 & 2.28 & 1.22 & 0.86 & 0.00 \\
\hline \multirow[t]{2}{*}{ Best bound } & 1.11 & 1.00 & 0.89 & 1.40 & 2.28 & 1.26 & 0.87 & 0.00 \\
\hline & \multicolumn{8}{|c|}{ Tests based on Imhof critical values } \\
\hline$\left|t\left(r_{1}\right)\right|$ & 5.09 & 2.30 & 0.38 & 19.57 & 36.59 & 34.07 & 52.75 & 54.88 \\
\hline$\left|\tilde{t}\left(r_{1}\right)\right|$ & 5.45 & 2.49 & 0.39 & 20.33 & 37.39 & 35.00 & 53.71 & 55.65 \\
\hline$\left|t\left(\hat{\rho}_{k}\right)\right|$ & 5.57 & 2.53 & 0.37 & 19.12 & 35.01 & 31.87 & 50.98 & 52.37 \\
\hline$\left|\bar{t}\left(\hat{\rho}_{k}\right)\right|$ & 5.20 & 2.47 & 0.35 & 19.44 & 35.74 & 32.61 & 51.34 & 53.54 \\
\hline & \multicolumn{8}{|c|}{ Tests based on global size correction } \\
\hline$\left|t\left(r_{1}\right)\right|$ & 0.02 & 0.00 & 0.00 & 0.70 & 5.07 & 0.04 & 0.00 & 0.00 \\
\hline$\left|\tilde{t}\left(r_{1}\right)\right|$ & 0.02 & 0.00 & 0.00 & 0.70 & 5.07 & 0.04 & 0.00 & 0.00 \\
\hline$\left|t\left(\hat{\rho}_{k}\right)\right|$ & 0.02 & 0.00 & 0.01 & 0.77 & 4.86 & 0.10 & 0.00 & 0.00 \\
\hline$\left|\bar{t}\left(\hat{\rho}_{k}\right)\right|$ & 0.03 & 0.00 & 0.00 & 0.81 & 5.10 & 0.01 & 0.00 & 0.00 \\
\hline & \multicolumn{8}{|c|}{ Tests based on model-specific size correction } \\
\hline$\left|t\left(r_{1}\right)\right|$ & 4.86 & 5.01 & 5.02 & 5.44 & 5.07 & 4.99 & 5.10 & 5.30 \\
\hline$\left|\tilde{t}\left(r_{1}\right)\right|$ & 4.86 & 5.01 & 5.02 & 5.44 & 5.07 & 4.99 & 5.10 & 5.30 \\
\hline$\left|t\left(\hat{\rho}_{k}\right)\right|$ & 4.80 & 4.73 & 5.09 & 5.30 & 4.86 & 5.25 & 4.90 & 5.17 \\
\hline$\left|\bar{t}\left(\hat{\rho}_{k}\right)\right|$ & 4.60 & 4.93 & 5.24 & 5.39 & 5.10 & 4.99 & 5.29 & 4.77 \\
\hline$v_{t} \sim$ Cauchy & \multicolumn{8}{|c|}{ Asymptotic tests and bounds } \\
\hline$\left|t\left(r_{1}\right)\right|$ & 2.47 & 2.43 & 1.70 & 4.82 & 18.68 & 6.27 & 23.44 & 34.36 \\
\hline$\left|\tilde{t}\left(r_{1}\right)\right|$ & 2.95 & 2.88 & 2.00 & 5.63 & 20.34 & 7.26 & 25.86 & 36.92 \\
\hline$\left|t\left(\hat{\rho}_{k}\right)\right|$ & 2.43 & 2.32 & 1.58 & 4.75 & 17.58 & 6.01 & 21.91 & 32.69 \\
\hline$\left|\bar{t}\left(\hat{\rho}_{k}\right)\right|$ & 2.88 & 2.62 & 1.84 & 5.30 & 19.15 & 6.67 & 23.93 & 34.90 \\
\hline$E_{11}$ & 1.10 & 1.33 & 1.35 & 1.37 & 2.84 & 1.39 & 1.68 & 0.01 \\
\hline Best bound & 1.16 & 1.36 & 1.36 & 1.40 & 2.84 & 1.43 & 1.68 & 0.01 \\
\hline & \multicolumn{8}{|c|}{ Tests based on Imhof critical values } \\
\hline$\left|t\left(r_{1}\right)\right|$ & 3.04 & 2.96 & 2.03 & 5.70 & 20.77 & 7.37 & 26.34 & 37.30 \\
\hline$\left|\tilde{t}\left(r_{1}\right)\right|$ & 3.21 & 3.17 & 2.06 & 5.88 & 21.41 & 7.62 & 27.13 & 37.97 \\
\hline$\left|t\left(\hat{\rho}_{k}\right)\right|$ & 2.95 & 3.10 & 1.80 & 5.62 & 19.91 & 7.15 & 24.78 & 35.58 \\
\hline$\left|\bar{t}\left(\hat{\rho}_{k}\right)\right|$ & 3.08 & 2.82 & 1.95 & 5.65 & 20.05 & 7.12 & 25.48 & 36.12 \\
\hline & \multicolumn{8}{|c|}{ Tests based on global size correction } \\
\hline$\left|t\left(r_{1}\right)\right|$ & 0.03 & 0.00 & 0.01 & 0.05 & 1.73 & 0.08 & 0.12 & 0.00 \\
\hline$\left|\tilde{t}\left(r_{1}\right)\right|$ & 0.03 & 0.00 & 0.01 & 0.05 & 1.73 & 0.08 & 0.12 & 0.00 \\
\hline$\left|t\left(\hat{\rho}_{k}\right)\right|$ & 0.02 & 0.00 & 0.01 & 0.05 & 1.60 & 0.10 & 0.12 & 0.00 \\
\hline$\left|\bar{t}\left(\hat{\rho}_{k}\right)\right|$ & 0.02 & 0.01 & 0.01 & 0.06 & 1.74 & 0.08 & 0.18 & 0.00 \\
\hline & \multicolumn{8}{|c|}{ Tests based on model-specific size correction } \\
\hline$\left|t\left(r_{1}\right)\right|$ & 5.17 & 5.15 & 5.10 & 5.11 & 5.24 & 5.43 & 4.94 & 5.01 \\
\hline$\left|\tilde{t}\left(r_{1}\right)\right|$ & 5.17 & 5.15 & 5.10 & 5.11 & 5.24 & 5.43 & 4.94 & 5.01 \\
\hline$\left|t\left(\hat{\rho}_{k}\right)\right|$ & 5.15 & 5.23 & 5.06 & 5.23 & 5.35 & 5.46 & 5.22 & 4.87 \\
\hline$\left|\bar{t}\left(\hat{\rho}_{k}\right)\right|$ & 5.11 & 5.04 & 5.09 & 5.19 & 5.22 & 5.48 & 5.10 & 5.28 \\
\hline
\end{tabular}


Table C.2. Empirical levels of serial dependence tests at nominal level $\alpha=0.05 ; n=60$

\begin{tabular}{|c|c|c|c|c|c|c|c|c|}
\hline$v_{t} \sim \mathrm{N}(0,1)$ & \multicolumn{8}{|c|}{ Asymptotic tests and bounds } \\
\hline Heteroskedasticity type & M1 & M2 & M3 & M4 & M5 & M6 & M7 & M8 \\
\hline$\left|t\left(r_{1}\right)\right|$ & 4.20 & 2.83 & 0.23 & 33.14 & 50.90 & 40.04 & 65.69 & 66.21 \\
\hline$\left|\tilde{t}\left(r_{1}\right)\right|$ & 4.89 & 3.22 & 0.26 & 34.31 & 51.92 & 41.38 & 66.58 & 67.12 \\
\hline$\left|t\left(\hat{\rho}_{k}\right)\right|$ & 4.24 & 2.81 & 0.24 & 32.44 & 49.98 & 39.13 & 65.08 & 65.57 \\
\hline$\left|\bar{t}\left(\hat{\rho}_{k}\right)\right|$ & 4.77 & 3.05 & 0.30 & 33.88 & 51.27 & 40.50 & 65.82 & 66.49 \\
\hline$E_{11}$ & 0.72 & 0.91 & 0.96 & 1.13 & 2.31 & 0.94 & 0.62 & 0.00 \\
\hline \multirow[t]{2}{*}{ Best bound } & 1.01 & 1.21 & 1.00 & 1.25 & 2.31 & 1.04 & 0.62 & 0.00 \\
\hline & \multicolumn{8}{|c|}{ Tests based on Imhof critical values } \\
\hline$\left|t\left(r_{1}\right)\right|$ & 4.99 & 3.29 & 0.27 & 34.55 & 52.05 & 41.57 & 66.69 & 67.23 \\
\hline$\left|\tilde{t}\left(r_{1}\right)\right|$ & 5.20 & 3.48 & 0.28 & 35.13 & 52.33 & 42.00 & 66.96 & 67.59 \\
\hline$\left|t\left(\hat{\rho}_{k}\right)\right|$ & 5.12 & 3.34 & 0.32 & 34.07 & 51.29 & 40.66 & 66.19 & 66.70 \\
\hline$\left|\bar{t}\left(\hat{\rho}_{k}\right)\right|$ & 4.95 & 3.16 & 0.31 & 34.36 & 51.85 & 41.09 & 66.29 & 66.91 \\
\hline & \multicolumn{8}{|c|}{ Tests based on global size correction } \\
\hline$\left|t\left(r_{1}\right)\right|$ & 0.00 & 0.00 & 0.00 & 0.70 & 5.07 & 0.01 & 0.00 & 0.00 \\
\hline$\left|\tilde{t}\left(r_{1}\right)\right|$ & 0.00 & 0.00 & 0.00 & 0.70 & 5.07 & 0.01 & 0.00 & 0.00 \\
\hline$\left|t\left(\hat{\rho}_{k}\right)\right|$ & 0.00 & 0.00 & 0.00 & 0.72 & 5.11 & 0.01 & 0.00 & 0.00 \\
\hline$\left|\bar{t}\left(\hat{\rho}_{k}\right)\right|$ & 0.00 & 0.00 & 0.00 & 0.66 & 5.07 & 0.01 & 0.00 & 0.00 \\
\hline & \multicolumn{8}{|c|}{ Tests based on model-specific size correction } \\
\hline$\left|t\left(r_{1}\right)\right|$ & 4.72 & 5.26 & 5.06 & 4.90 & 5.07 & 5.53 & 5.19 & 4.83 \\
\hline$\left|\tilde{t}\left(r_{1}\right)\right|$ & 4.72 & 5.26 & 5.06 & 4.90 & 5.07 & 5.53 & 5.19 & 4.83 \\
\hline$\left|t\left(\hat{\rho}_{k}\right)\right|$ & 4.59 & 5.09 & 5.42 & 4.92 & 5.11 & 5.22 & 5.34 & 4.96 \\
\hline$\left|\bar{t}\left(\hat{\rho}_{k}\right)\right|$ & 4.67 & 5.28 & 5.09 & 5.03 & 5.07 & 5.60 & 5.16 & 4.95 \\
\hline$v_{t} \sim$ Cauchy & \multicolumn{8}{|c|}{ Asymptotic tests and bounds } \\
\hline$\left|t\left(r_{1}\right)\right|$ & 2.88 & 2.92 & 2.01 & 10.19 & 30.90 & 6.03 & 30.08 & 48.90 \\
\hline$\left|\tilde{t}\left(r_{1}\right)\right|$ & 3.07 & 3.09 & 2.18 & 10.70 & 31.61 & 6.41 & 30.75 & 49.77 \\
\hline$\left|t\left(\hat{\rho}_{k}\right)\right|$ & 2.84 & 2.88 & 2.07 & 10.11 & 30.30 & 5.75 & 29.55 & 48.09 \\
\hline$\left|\bar{t}\left(\hat{\rho}_{k}\right)\right|$ & 2.95 & 3.13 & 2.06 & 10.60 & 31.19 & 6.24 & 30.39 & 49.12 \\
\hline$E_{11}$ & 1.15 & 1.08 & 1.10 & 1.43 & 2.95 & 1.11 & 1.45 & 0.00 \\
\hline Best bound & 1.24 & 1.10 & 1.16 & 1.48 & 2.95 & 1.14 & 1.45 & 0.00 \\
\hline & \multicolumn{8}{|c|}{ Tests based on Imhof critical values } \\
\hline$\left|t\left(r_{1}\right)\right|$ & 3.12 & 3.12 & 2.21 & 10.83 & 31.75 & 6.47 & 30.90 & 49.87 \\
\hline$\left|\tilde{t}\left(r_{1}\right)\right|$ & 3.21 & 3.19 & 2.28 & 11.12 & 31.93 & 6.59 & 31.12 & 50.17 \\
\hline$\left|t\left(\hat{\rho}_{k}\right)\right|$ & 3.09 & 3.09 & 2.19 & 10.82 & 31.25 & 6.25 & 30.51 & 49.13 \\
\hline$\left|\bar{t}\left(\hat{\rho}_{k}\right)\right|$ & 3.07 & 3.20 & 2.19 & 10.81 & 31.57 & 6.36 & 30.81 & 49.55 \\
\hline & \multicolumn{8}{|c|}{ Tests based on global size correction } \\
\hline$\left|t\left(r_{1}\right)\right|$ & 0.00 & 0.00 & 0.00 & 0.09 & 1.61 & 0.01 & 0.06 & 0.00 \\
\hline$\left|\tilde{t}\left(r_{1}\right)\right|$ & 0.00 & 0.00 & 0.00 & 0.09 & 1.61 & 0.01 & 0.06 & 0.00 \\
\hline$\left|t\left(\hat{\rho}_{k}\right)\right|$ & 0.00 & 0.00 & 0.00 & 0.09 & 1.66 & 0.01 & 0.07 & 0.00 \\
\hline$\left|\bar{t}\left(\hat{\rho}_{k}\right)\right|$ & 0.00 & 0.00 & 0.00 & 0.11 & 1.58 & 0.01 & 0.05 & 0.00 \\
\hline & \multicolumn{8}{|c|}{ Tests based on model-specific size correction } \\
\hline$\left|t\left(r_{1}\right)\right|$ & 5.12 & 4.99 & 4.72 & 5.02 & 5.42 & 5.30 & 4.83 & 4.65 \\
\hline$\left|\tilde{t}\left(r_{1}\right)\right|$ & 5.12 & 4.99 & 4.72 & 5.02 & 5.42 & 5.30 & 4.83 & 4.65 \\
\hline$\left|t\left(\hat{\rho}_{k}\right)\right|$ & 5.03 & 4.85 & 4.65 & 4.95 & 5.35 & 5.22 & 4.85 & 5.03 \\
\hline$\left|\bar{t}\left(\hat{\rho}_{k}\right)\right|$ & 5.07 & 4.98 & 4.71 & 4.99 & 5.35 & 5.33 & 4.61 & 4.93 \\
\hline
\end{tabular}


Table C.3. Empirical levels of serial dependence tests at nominal level $\alpha=0.05 ; n=100$

\begin{tabular}{|c|c|c|c|c|c|c|c|c|}
\hline$v_{t} \sim \mathrm{N}(0,1)$ & \multicolumn{8}{|c|}{ Asymptotic tests and bounds } \\
\hline Heteroskedasticity type & M1 & M2 & M3 & M4 & M5 & M6 & M7 & M8 \\
\hline$\left|t\left(r_{1}\right)\right|$ & 4.63 & 3.37 & 0.43 & 45.62 & 61.60 & 43.65 & 73.56 & 73.98 \\
\hline$\left|\tilde{t}\left(r_{1}\right)\right|$ & 4.92 & 3.60 & 0.46 & 46.30 & 62.11 & 44.63 & 73.98 & 74.29 \\
\hline$\left|t\left(\hat{\rho}_{k}\right)\right|$ & 4.73 & 3.34 & 0.47 & 45.34 & 61.08 & 43.41 & 73.14 & 73.53 \\
\hline$\left|\bar{t}\left(\hat{\rho}_{k}\right)\right|$ & 4.93 & 3.60 & 0.46 & 45.96 & 61.78 & 44.32 & 73.68 & 74.08 \\
\hline$E_{11}$ & 0.79 & 0.84 & 0.86 & 1.14 & 2.48 & 0.79 & 0.60 & 0.00 \\
\hline \multirow[t]{2}{*}{ Best bound } & 0.99 & 1.17 & 0.93 & 1.21 & 2.48 & 0.87 & 0.60 & 0.00 \\
\hline & \multicolumn{8}{|c|}{ Tests based on global size correction } \\
\hline$\left|t\left(r_{1}\right)\right|$ & 0.00 & 0.00 & 0.00 & 0.70 & 5.15 & 0.00 & 0.00 & 0.00 \\
\hline$\left|\tilde{t}\left(r_{1}\right)\right|$ & 0.00 & 0.00 & 0.00 & 0.70 & 5.15 & 0.00 & 0.00 & 0.00 \\
\hline$\left|t\left(\hat{\rho}_{k}\right)\right|$ & 0.00 & 0.00 & 0.00 & 0.75 & 5.19 & 0.00 & 0.00 & 0.00 \\
\hline$\left|\bar{t}\left(\hat{\rho}_{k}\right)\right|$ & 0.00 & 0.00 & 0.00 & 0.74 & 5.09 & 0.00 & 0.00 & 0.00 \\
\hline & \multicolumn{8}{|c|}{ Tests based on model-specific size correction } \\
\hline$\left|t\left(r_{1}\right)\right|$ & 4.90 & 5.34 & 4.82 & 4.95 & 5.15 & 5.20 & 4.93 & 4.92 \\
\hline$\left|\tilde{t}\left(r_{1}\right)\right|$ & 4.90 & 5.34 & 4.82 & 4.95 & 5.15 & 5.20 & 4.93 & 4.92 \\
\hline$\left|t\left(\hat{\rho}_{k}\right)\right|$ & 4.83 & 5.36 & 4.66 & 4.98 & 5.19 & 5.06 & 4.82 & 5.11 \\
\hline$\left|\bar{t}\left(\hat{\rho}_{k}\right)\right|$ & 4.88 & 5.39 & 4.75 & 4.97 & 5.09 & 5.22 & 4.95 & 4.96 \\
\hline$v_{t} \sim$ Cauchy & \multicolumn{8}{|c|}{ Asymptotic tests and bounds } \\
\hline$\left|t\left(r_{1}\right)\right|$ & 2.96 & 2.98 & 2.25 & 16.40 & 38.37 & 4.65 & 31.18 & 56.84 \\
\hline$\left|\tilde{t}\left(r_{1}\right)\right|$ & 3.08 & 3.09 & 2.36 & 16.76 & 38.92 & 4.81 & 31.58 & 57.35 \\
\hline$\left|t\left(\hat{\rho}_{k}\right)\right|$ & 2.95 & 2.98 & 2.25 & 16.22 & 38.23 & 4.66 & 30.78 & 56.68 \\
\hline$\left|\bar{t}\left(\hat{\rho}_{k}\right)\right|$ & 3.03 & 3.01 & 2.28 & 16.36 & 38.66 & 4.78 & 31.09 & 56.92 \\
\hline$E_{11}$ & 1.10 & 1.19 & 1.08 & 1.52 & 2.85 & 1.32 & 1.38 & 0.00 \\
\hline Best bound & 1.19 & 1.23 & 1.10 & 1.52 & 2.85 & 1.35 & 1.38 & 0.00 \\
\hline & \multicolumn{8}{|c|}{ Tests based on global size correction } \\
\hline$\left|t\left(r_{1}\right)\right|$ & 0.00 & 0.00 & 0.01 & 0.07 & 1.55 & 0.00 & 0.04 & 0.00 \\
\hline$\left|\tilde{t}\left(r_{1}\right)\right|$ & 0.00 & 0.00 & 0.01 & 0.07 & 1.55 & 0.00 & 0.04 & 0.00 \\
\hline$\left|t\left(\hat{\rho}_{k}\right)\right|$ & 0.00 & 0.00 & 0.01 & 0.07 & 1.57 & 0.00 & 0.04 & 0.00 \\
\hline$\left|\bar{t}\left(\hat{\rho}_{k}\right)\right|$ & 0.00 & 0.00 & 0.01 & 0.08 & 1.58 & 0.00 & 0.04 & 0.00 \\
\hline & \multicolumn{8}{|c|}{ Tests based on model-specific size correction } \\
\hline$\left|t\left(r_{1}\right)\right|$ & 4.97 & 5.13 & 4.56 & 5.51 & 4.86 & 4.77 & 4.73 & 4.68 \\
\hline$\left|\tilde{t}\left(r_{1}\right)\right|$ & 4.97 & 5.13 & 4.56 & 5.51 & 4.86 & 4.77 & 4.73 & 4.68 \\
\hline$\left|t\left(\hat{\rho}_{k}\right)\right|$ & 5.02 & 5.08 & 4.53 & 5.39 & 4.92 & 4.81 & 4.57 & 4.70 \\
\hline$\left|\bar{t}\left(\hat{\rho}_{k}\right)\right|$ & 4.86 & 4.99 & 4.53 & 5.45 & 4.92 & 4.82 & 4.81 & 4.85 \\
\hline
\end{tabular}

Note - For $n=100$, the Imhof algorithm became numerically unstable. Further, in the case where the latter applies (i.i.d. Gaussian observations), it is apparent from the above results that the asymptotic critical values are practically exact. Consequently, for $n=100$, we will not report simulation results based on Imhof critical values. 
Table C.4. Critical values for size-corrected tests with level $\alpha=0.05$

a. Model-specific critical values

\begin{tabular}{|c|c|c|c|c|c|c|c|c|}
\hline & \multicolumn{8}{|c|}{$n=30, v_{t} \sim \mathrm{N}(0,1)$} \\
\hline & M1 & M2 & M3 & M4 & M5 & M6 & M7 & M8 \\
\hline$\left|t\left(r_{1}\right)\right|$ & 1.878465 & 1.589726 & 0.669122 & 2.664882 & 3.442610 & 2.693328 & 2.777222 & 2.775027 \\
\hline$\left|\tilde{t}\left(r_{1}\right)\right|$ & 1.970150 & 1.667319 & 0.701781 & 2.794952 & 3.610639 & 2.824786 & 2.912775 & 2.910472 \\
\hline$\left|t\left(\hat{\rho}_{k}\right)\right|$ & 1.909817 & 1.622474 & 0.712442 & 2.635794 & 3.378111 & 2.636964 & 2.739768 & 2.753799 \\
\hline \multirow[t]{2}{*}{$\left|\bar{t}\left(\hat{\rho}_{k}\right)\right|$} & 1.963357 & 1.656611 & 0.696682 & 2.741489 & 3.517594 & 2.744493 & 2.844584 & 2.855707 \\
\hline & \multicolumn{8}{|c|}{$n=30, v_{t} \sim$ Cauchy } \\
\hline$\left|t\left(r_{1}\right)\right|$ & 1.581895 & 1.573432 & 1.187341 & 1.922169 & 2.884731 & 2.040721 & 2.675898 & 2.763140 \\
\hline$\left|\tilde{t}\left(r_{1}\right)\right|$ & 1.659106 & 1.650230 & 1.245293 & 2.015987 & 3.025531 & 2.140327 & 2.806505 & 2.898006 \\
\hline$\left|t\left(\hat{\rho}_{k}\right)\right|$ & 1.590586 & 1.577626 & 1.205998 & 1.905994 & 2.813909 & 2.007700 & 2.589812 & 2.709071 \\
\hline \multirow[t]{2}{*}{$\left|\bar{t}\left(\hat{\rho}_{k}\right)\right|$} & 1.639214 & 1.618274 & 1.232452 & 1.973277 & 2.945075 & 2.088720 & 2.703483 & 2.799635 \\
\hline & \multicolumn{8}{|c|}{$n=60, v_{t} \sim \mathrm{N}(0,1)$} \\
\hline$\left|t\left(r_{1}\right)\right|$ & 1.923251 & 1.701257 & 0.817359 & 3.731782 & 4.833327 & 3.421299 & 3.883162 & 3.893266 \\
\hline$\left|\tilde{t}\left(r_{1}\right)\right|$ & 1.970746 & 1.743270 & 0.837543 & 3.823938 & 4.952686 & 789 & 3.979057 & 3.989411 \\
\hline$\left|t\left(\hat{\rho}_{k}\right)\right|$ & 1.940905 & 1.715555 & 0.837857 & 3.698896 & 4.775711 & 3.378436 & 3.833714 & 3.860026 \\
\hline \multirow[t]{2}{*}{$\left|\bar{t}\left(\hat{\rho}_{k}\right)\right|$} & 1.971420 & 1.742014 & 0.835665 & 3.773769 & 4.899128 & 3.451324 & 3.923278 & 3.943605 \\
\hline & \multicolumn{8}{|c|}{$n=60, v_{t} \sim$ Cauchy } \\
\hline$\left|t\left(r_{1}\right)\right|$ & 1.587317 & 1.58 & 1.278107 & 2.591782 & 4.039616 & 2.06 & 3.573439 & 3.874248 \\
\hline$\left|\tilde{t}\left(r_{1}\right)\right|$ & 1.626516 & 1.624246 & 1.309670 & 2.655786 & 4.139374 & 2.115484 & 3.661685 & 3.969923 \\
\hline$\left|t\left(\hat{\rho}_{k}\right)\right|$ & 1.588293 & 1.582660 & 1.284447 & 2.566254 & 3.994436 & 2.043048 & 3.511895 & 3.785808 \\
\hline \multirow[t]{2}{*}{$\left|\bar{t}\left(\hat{\rho}_{k}\right)\right|$} & 1.617556 & 1.611527 & 1.295008 & 2.614433 & 4.086018 & 2.091978 & 3.597454 & 3.874049 \\
\hline & \multicolumn{8}{|c|}{$n=100, v_{t} \sim \mathrm{N}(0,1)$} \\
\hline$\left|t\left(r_{1}\right)\right|$ & 1.936850 & 1.775824 & 0.930278 & 4.799030 & 6.186322 & 4.009946 & 4.981868 & 5.009529 \\
\hline$\left|\tilde{t}\left(r_{1}\right)\right|$ & 1.965688 & 1.802265 & 0.944129 & 4.870483 & 6.278432 & 4.069651 & 5.056044 & 5.084116 \\
\hline$\left|t\left(\hat{\rho}_{k}\right)\right|$ & 1.948487 & 1.783135 & 0.940931 & 4.762880 & 6.149153 & 3.977697 & 4.935454 & 4.964416 \\
\hline \multirow[t]{2}{*}{$\left|\bar{t}\left(\hat{\rho}_{k}\right)\right|$} & 1.966353 & 1.799861 & 0.942239 & 4.831045 & 6.237931 & 4.033077 & 5.006537 & 5.031303 \\
\hline & \multicolumn{8}{|c|}{$n=100, v_{t} \sim$ Cauchy } \\
\hline$\left|t\left(r_{1}\right)\right|$ & 1.552881 & 1.536918 & 1.289574 & 3.329494 & 5.223522 & 1.935397 & 4.294783 & 4.987568 \\
\hline$\left|\tilde{t}\left(r_{1}\right)\right|$ & 1.576002 & 1.559801 & 1.308775 & 3.379067 & 5.301296 & 1.964213 & 4.358729 & 5.061829 \\
\hline $\mid t\left(\hat{\rho}_{k}\right)$ & 1.556296 & 1.540831 & 1.289064 & 3.303165 & 5.185667 & 1.929854 & 4.252619 & 4.918678 \\
\hline & 1.579980 & 1.561243 & 1.305322 & 3.347292 & 5.253193 & 1.951267 & 4.310394 & 4.993854 \\
\hline
\end{tabular}

b. Imhof and global critical values

\begin{tabular}{|c|c|c|c|c|c|}
\cline { 2 - 6 } \multicolumn{1}{c|}{} & \multicolumn{2}{c|}{$n=30$} & \multicolumn{2}{c|}{$n=60$} & $n=100$ \\
\cline { 2 - 6 } \multicolumn{1}{c|}{} & Imhof & Global & Imhof & Global & Global \\
\hline$\left|t\left(r_{1}\right)\right|$ & 1.852781 & 3.442610 & 1.905275 & 4.833327 & 6.186322 \\
$\left|\tilde{t}\left(r_{1}\right)\right|$ & 1.913544 & 3.610639 & 1.936770 & 4.952686 & 6.278432 \\
$\left|t\left(\hat{\rho}_{k}\right)\right|$ & 1.852781 & 3.378111 & 1.905275 & 4.775711 & 6.149153 \\
$\left|\bar{t}\left(\hat{\rho}_{k}\right)\right|$ & 1.913544 & 3.517594 & 1.936770 & 4.899128 & 6.237931 \\
\hline
\end{tabular}


Table C.5. Empirical powers of serial dependence tests at level $\alpha=0.05$ $X_{t}=0.2 X_{t-1}+u_{t}, v_{t} \sim \mathrm{N}(0,1)$

\begin{tabular}{|c|c|c|c|c|c|c|c|c|}
\hline Sample size: $n=30$ & \multicolumn{8}{|c|}{ Asymptotic tests and bounds } \\
\hline Test $\backslash$ Model & M1 & M2 & M3 & M4 & M5 & M6 & M7 & M8 \\
\hline$E_{11}$ & 4.81 & 7.15 & 16.25 & 3.89 & 3.51 & 6.93 & 19.51 & 48.06 \\
\hline Best bound & 5.66 & 7.54 & 16.27 & 4.09 & 3.51 & 7.03 & 19.52 & 48.06 \\
\hline & \multicolumn{8}{|c|}{ Tests based on global size correction } \\
\hline$\left|t\left(r_{1}\right)\right|$ & 0.22 & 0.02 & 0.01 & 2.43 & 7.77 & 1.40 & 0.19 & 0.00 \\
\hline$\left|\tilde{t}\left(r_{1}\right)\right|$ & 0.22 & 0.02 & 0.01 & 2.43 & 7.77 & 1.40 & 0.19 & 0.00 \\
\hline$\left|t\left(\hat{\rho}_{k}\right)\right|$ & 0.12 & 0.02 & 0.01 & 1.59 & 6.61 & 0.58 & 0.03 & 0.00 \\
\hline$\left|\bar{t}\left(\hat{\rho}_{k}\right)\right|$ & 0.37 & 0.05 & 0.03 & 2.68 & 8.57 & 2.05 & 0.60 & 0.00 \\
\hline & \multicolumn{8}{|c|}{ Tests based on model-specific size correction } \\
\hline$\left|t\left(r_{1}\right)\right|$ & 18.71 & 23.05 & 95.57 & 11.30 & 7.77 & 17.21 & 24.77 & 25.27 \\
\hline$\left|\tilde{t}\left(r_{1}\right)\right|$ & 18.71 & 23.05 & 95.57 & 11.30 & 7.77 & 17.21 & 24.77 & 25.27 \\
\hline$\left|t\left(\hat{\rho}_{k}\right)\right|$ & 12.36 & 13.78 & 86.81 & 8.78 & 6.61 & 12.88 & 20.38 & 20.32 \\
\hline$\left|\bar{t}\left(\hat{\rho}_{k}\right)\right|$ & 17.73 & 21.54 & 94.54 & 11.52 & 8.57 & 18.21 & 24.93 & 25.26 \\
\hline Sample size: $n=60$ & \multicolumn{8}{|c|}{ Asymptotic tests and bounds } \\
\hline$E_{11}$ & 11.54 & 13.92 & 22.85 & 4.00 & 3.54 & 11.69 & 21.85 & 49.02 \\
\hline Best bound & 13.71 & 15.26 & 23.06 & 4.21 & 3.55 & 12.13 & 21.85 & 49.02 \\
\hline & \multicolumn{8}{|c|}{ Tests based on global size correction } \\
\hline$\left|t\left(r_{1}\right)\right|$ & 0.00 & 0.00 & 0.00 & 2.46 & 8.00 & 0.19 & 0.11 & 0.00 \\
\hline$\left|\tilde{t}\left(r_{1}\right)\right|$ & 0.00 & 0.00 & 0.00 & 2.46 & 8.00 & 0.19 & 0.11 & 0.00 \\
\hline$\left|t\left(\hat{\rho}_{k}\right)\right|$ & 0.00 & 0.00 & 0.00 & 2.19 & 7.54 & 0.09 & 0.00 & 0.00 \\
\hline$\left|\bar{t}\left(\hat{\rho}_{k}\right)\right|$ & 0.01 & 0.00 & 0.00 & 2.73 & 8.33 & 0.32 & 0.20 & 0.00 \\
\hline & \multicolumn{8}{|c|}{ Tests based on model-specific size correction } \\
\hline$\left|t\left(r_{1}\right)\right|$ & 33.22 & 41.38 & 96.86 & 10.76 & 8.00 & 17.50 & 24.79 & 25.42 \\
\hline$\left|\tilde{t}\left(r_{1}\right)\right|$ & 33.22 & 41.3 & 96.86 & 10.76 & 8.00 & 17.50 & 24.79 & 25.42 \\
\hline$\left|t\left(\hat{\rho}_{k}\right)\right|$ & 27.21 & 33.3 & 95.13 & 9.6 & 7.54 & 15.48 & 22.97 & 23.38 \\
\hline$\left|\bar{t}\left(\hat{\rho}_{k}\right)\right|$ & 32.26 & 39.92 & 96.50 & 11.09 & 8.33 & 17.87 & 25.20 & 25.75 \\
\hline Sample size: $n=100$ & \multicolumn{8}{|c|}{ Asymptotic tests and bounds } \\
\hline$E_{11}$ & 22.61 & 24.80 & 30.30 & 4.04 & 3.67 & 19.70 & 24.94 & 47.42 \\
\hline Best bound & 25.76 & 26.98 & 30.60 & 4.22 & 3.67 & 20.38 & 24.96 & 47.42 \\
\hline & \multicolumn{8}{|c|}{ Tests based on global size correction } \\
\hline$\left|t\left(r_{1}\right)\right|$ & 0.00 & 0.00 & 0.00 & 2.52 & 8.26 & 0.03 & 0.10 & 0.00 \\
\hline$\left|\tilde{t}\left(r_{1}\right)\right|$ & 0.00 & 0.0 & 0.00 & 2.52 & 8.26 & 0.03 & 0.10 & 0.00 \\
\hline$\left|t\left(\hat{\rho}_{k}\right)\right|$ & 0.00 & 0.00 & 0.00 & 2.28 & 8.01 & 0.02 & 0.03 & 0.00 \\
\hline$\left|\bar{t}\left(\hat{\rho}_{k}\right)\right|$ & 0.00 & 0.00 & 0.00 & 2.57 & 8.46 & 0.04 & 0.17 & 0.00 \\
\hline & \multicolumn{8}{|c|}{ Tests based on model-specific size correction } \\
\hline$\left|t\left(r_{1}\right)\right|$ & 50.14 & 58.93 & 98.31 & 11.65 & 8.26 & 18.16 & 24.05 & 24.43 \\
\hline$\left|\tilde{t}\left(r_{1}\right)\right|$ & 50.14 & 58.93 & 98.31 & 11.65 & 8.26 & 18.16 & 24.05 & 24.43 \\
\hline$\left|t\left(\hat{\rho}_{k}\right)\right|$ & 44.96 & 52.77 & 97.50 & 11.03 & 8.01 & 17.02 & 23.27 & 23.62 \\
\hline$\left|\bar{t}\left(\hat{\rho}_{k}\right)\right|$ & 49.03 & 58.08 & 98.11 & 11.90 & 8.46 & 18.43 & 24.39 & 24.87 \\
\hline
\end{tabular}


Table C.6. Empirical powers of serial dependence tests at level $\alpha=0.05$

$$
X_{t}=0.2 X_{t-1}+u_{t}, v_{t} \sim \text { Cauchy }
$$

\begin{tabular}{|c|c|c|c|c|c|c|c|c|}
\hline \multirow{2}{*}{$\begin{array}{c}\text { Sample size: } n=30 \\
\text { Test } \backslash \text { Model }\end{array}$} & \multicolumn{8}{|c|}{ Asymptotic tests and bounds } \\
\hline & M1 & M2 & M3 & M4 & M5 & M6 & M7 & M8 \\
\hline$E_{11}$ & 13.37 & 14.59 & 18.76 & 10.96 & 7.29 & 14.31 & 19.78 & 44.49 \\
\hline Best bound & 13.59 & 14.72 & 18.79 & 11.05 & 7.29 & 14.42 & 19.78 & 44.49 \\
\hline & \multicolumn{8}{|c|}{ Tests based on global size correction } \\
\hline$\left|t\left(r_{1}\right)\right|$ & 0.08 & 0.11 & 0.06 & 0.50 & 2.98 & 0.33 & 0.66 & 0.00 \\
\hline$\left|\tilde{t}\left(r_{1}\right)\right|$ & 0.08 & 0.11 & 0.06 & 0.50 & 2.98 & 0.33 & 0.66 & 0.00 \\
\hline$\left|t\left(\hat{\rho}_{k}\right)\right|$ & 0.03 & 0.08 & 0.02 & 0.33 & 2.36 & 0.20 & 0.25 & 0.00 \\
\hline$\left|\bar{t}\left(\hat{\rho}_{k}\right)\right|$ & 0.10 & 0.13 & 0.06 & 0.69 & 3.48 & 0.34 & 0.83 & 0.00 \\
\hline & \multicolumn{8}{|c|}{ Tests based on model-specific size correction } \\
\hline$\left|t\left(r_{1}\right)\right|$ & 16.03 & 16.16 & 28.45 & 12.84 & 8.59 & 11.51 & 14.08 & 16.75 \\
\hline$\left|\tilde{t}\left(r_{1}\right)\right|$ & 16.03 & 16.16 & 28.45 & 12.84 & 8.59 & 11.51 & 14.08 & 16.75 \\
\hline$\left|t\left(\hat{\rho}_{k}\right)\right|$ & 10.95 & 10.72 & 13.27 & 9.49 & 7.37 & 9.01 & 11.67 & 13.63 \\
\hline$\left|\bar{t}\left(\hat{\rho}_{k}\right)\right|$ & 15.71 & 15.91 & 27.22 & 12.54 & 8.99 & 11.70 & 14.90 & 17.42 \\
\hline Sample size: $n=60$ & \multicolumn{8}{|c|}{ Asymptotic tests and bounds } \\
\hline$E_{11}$ & 26.04 & 25.75 & 28.16 & 13.24 & 7.40 & 23.49 & 25.78 & 44.41 \\
\hline \multirow[t]{2}{*}{ Best bound } & 26.32 & 26.09 & 28.22 & 13.35 & 7.40 & 23.76 & 25.79 & 44.41 \\
\hline & \multicolumn{8}{|c|}{ Tests based on global size correction } \\
\hline$\left|t\left(r_{1}\right)\right|$ & 0.01 & 0.00 & 0.00 & 0.32 & 3.16 & 0.06 & 0.33 & 0.00 \\
\hline$\left|\tilde{t}\left(r_{1}\right)\right|$ & 0.01 & 0.00 & 0.00 & 0.32 & 3.16 & 0.06 & 0.33 & 0.00 \\
\hline$\left|t\left(\hat{\rho}_{k}\right)\right|$ & 0.00 & 0.00 & 0.00 & 0.27 & 2.88 & 0.04 & 0.25 & 0.00 \\
\hline$\left|\bar{t}\left(\hat{\rho}_{k}\right)\right|$ & 0.02 & 0.00 & 0.00 & 0.38 & 3.37 & 0.05 & 0.36 & 0.00 \\
\hline & \multicolumn{8}{|c|}{ Tests based on model-specific size correction } \\
\hline$\left|t\left(r_{1}\right)\right|$ & 43.25 & 42.27 & 84.59 & 13.22 & 8.66 & 17.82 & 12.34 & 17.18 \\
\hline$\left|\tilde{t}\left(r_{1}\right)\right|$ & 43.25 & 42.27 & 84.59 & 13.22 & 8.66 & 17.82 & 12.34 & 17.18 \\
\hline$\left|t\left(\hat{\rho}_{k}\right)\right|$ & 29.29 & 27. & 71.23 & 11. & 8.14 & 14.48 & 11 & 16.55 \\
\hline$\left|\bar{t}\left(\hat{\rho}_{k}\right)\right|$ & 41.73 & 41.69 & 82.53 & 13.23 & 9.00 & 17.74 & 12.50 & 18.09 \\
\hline Sample size: $n=100$ & \multicolumn{8}{|c|}{ Asymptotic tests and bounds } \\
\hline$E_{11}$ & 37.85 & 38.89 & 39.03 & 13.08 & 7.88 & 35.69 & 31.75 & 44.79 \\
\hline \multirow[t]{2}{*}{ Best bound } & 38.19 & 39.30 & 39.23 & 13.29 & 7.88 & 35.97 & 31.77 & 44.79 \\
\hline & \multicolumn{8}{|c|}{ Tests based on global size correction } \\
\hline$\left|t\left(r_{1}\right)\right|$ & 0.00 & 0.01 & 0.01 & 0.33 & 3.45 & 0.01 & 0.08 & 0.00 \\
\hline$\left|\tilde{t}\left(r_{1}\right)\right|$ & 0.00 & 0.01 & 0.01 & 0.33 & 3.45 & 0.01 & 0.08 & 0.00 \\
\hline$\left|t\left(\hat{\rho}_{k}\right)\right|$ & 0.00 & 0.01 & 0.01 & 0.26 & 3.29 & 0.01 & 0.08 & 0.00 \\
\hline$\left|\bar{t}\left(\hat{\rho}_{k}\right)\right|$ & 0.00 & 0.01 & 0.01 & 0.32 & 3.60 & 0.02 & 0.09 & 0.00 \\
\hline & \multicolumn{8}{|c|}{ Tests based on model-specific size correction } \\
\hline$\left|t\left(r_{1}\right)\right|$ & 84.26 & 85.02 & 94.12 & 13.14 & 8.62 & 61.29 & 10.65 & 16.92 \\
\hline$\left|\tilde{t}\left(r_{1}\right)\right|$ & 84.26 & 85.02 & 94.12 & 13.14 & 8.62 & 61.29 & 10.65 & 16.92 \\
\hline$\left|t\left(\hat{\rho}_{k}\right)\right|$ & 79.03 & 80.30 & 92.49 & 11.96 & 8.28 & 41.66 & 10.13 & 16.29 \\
\hline$\left|\bar{t}\left(\hat{\rho}_{k}\right)\right|$ & 83.09 & 83.92 & 93.74 & 13.10 & 8.80 & 59.22 & 10.86 & 17.42 \\
\hline
\end{tabular}


Table C.7. Empirical powers of serial dependence tests at level $\alpha=0.05$

$$
X_{t}=0.3 X_{t-1}+u_{t}, v_{t} \sim \mathrm{N}(0,1)
$$

\begin{tabular}{|c|c|c|c|c|c|c|c|c|}
\hline Sample size: $n=30$ & \multicolumn{8}{|c|}{ Asymptotic tests and bounds } \\
\hline Test $\backslash$ Model & M1 & M2 & M3 & M4 & M5 & M6 & M7 & M8 \\
\hline$E_{11}$ & 13.26 & 16.35 & 31.86 & 7.94 & 4.66 & 14.55 & 26.86 & 54.08 \\
\hline Best $b$ & & 17.01 & 31.91 & 8.29 & 4.66 & 14.70 & 26.86 & 54.08 \\
\hline & \multicolumn{8}{|c|}{ Tests based on global size correction } \\
\hline$\left|t\left(r_{1}\right)\right|$ & 0.99 & 0.46 & 0.05 & 5.66 & 11.90 & 6.58 & 14.74 & 15.39 \\
\hline$\left|\tilde{t}\left(r_{1}\right)\right|$ & 0.99 & 0.46 & 0.05 & 5.66 & 90 & 6.58 & 14.74 & 15.39 \\
\hline & 0.57 & 0.25 & 0.03 & 4.07 & 10.53 & 3.33 & 4.79 & 5.67 \\
\hline $\bar{t}\left(\hat{\rho}_{k}\right)$ & 1.33 & 0.59 & 0.09 & 6.10 & 13.60 & 7.63 & 16.34 & 17.11 \\
\hline & \multicolumn{8}{|c|}{ Tests based on model-specific size correction } \\
\hline$\left|t\left(r_{1}\right)\right|$ & .31 & 51.69 & 98.85 & 19.59 & 11.90 & 26.93 & 32.93 & 33.52 \\
\hline$\left|\tilde{t}\left(r_{1}\right)\right|$ & 31 & 51.69 & 98.85 & 19.59 & 11.90 & 26.93 & 32.93 & 33.52 \\
\hline$\left|t\left(\hat{\rho}_{k}\right)\right|$ & 6.56 & 34.05 & 97.60 & 15.33 & 10.53 & 21.96 & 28.64 & 28.72 \\
\hline$\left|\bar{t}\left(\hat{\rho}_{k}\right)\right|$ & 4.95 & 47.21 & 98.58 & 19.64 & 13.60 & 26.89 & 32.84 & 33.09 \\
\hline Sample size: $n=60$ & \multicolumn{8}{|c|}{ Asymptotic tests and bounds } \\
\hline$E_{1}$ & 01 & 35.04 & 42.67 & 8.44 & 4.32 & 28.95 & 31.22 & $53.5 \mathrm{c}$ \\
\hline Best b & 76 & 36.99 & 42.85 & 8.72 & 4.32 & 29.61 & 31.24 & 53.5 \\
\hline & \multicolumn{8}{|c|}{ Tests based on global size correction } \\
\hline$t(r$ & 0.06 & 0.06 & 0.01 & 5.77 & 12.11 & 3.15 & 14.27 & 15.23 \\
\hline$\left|\tilde{t}\left(r_{1}\right)\right|$ & 0.0 & 0.06 & 0.01 & & & 3.15 & 14.27 & 15.23 \\
\hline$\left|t\left(\hat{\rho}_{k}\right)\right|$ & 0.0 & 0.03 & 0.00 & 5. & 11 & 2.18 & 10.81 & 12.5 \\
\hline$\left|\bar{t}\left(\hat{\rho}_{k}\right)\right|$ & 0 & 0.05 & 0.00 & 6.20 & 12.75 & 3.64 & 15.29 & 16.16 \\
\hline & \multicolumn{8}{|c|}{ Tests based on model-specific size correction } \\
\hline$\left|t\left(r_{1}\right)\right|$ & 0 & 77.02 & 99.50 & 19.82 & 12.11 & 29.79 & 32.82 & 32.97 \\
\hline$\left|\tilde{t}\left(r_{1}\right)\right|$ & 4.01 & 77.02 & 99.50 & 19 & 12. & 29.79 & 32.82 & 32.97 \\
\hline$\left|t\left(\hat{\rho}_{k}\right)\right|$ & 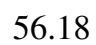 & 69.71 & 99.15 & 17. & 11. & 27.27 & 31.40 & 31.03 \\
\hline$\left|\bar{t}\left(\hat{\rho}_{k}\right)\right|$ & & 75.24 & 99.40 & 20.29 & 12.75 & 30.09 & 33.00 & 33.03 \\
\hline Sample size: $n=10$ & \multicolumn{8}{|c|}{ Asymptotic tests and bounds } \\
\hline$E_{11}$ & 61.0 & 57.10 & 51.15 & 7.7 & 4.90 & 43.02 & 35.33 & 55.01 \\
\hline Best bound & 6 & 59.56 & 51.41 & 8.10 & 4.90 & 43.88 & 35.36 & 55.01 \\
\hline & \multicolumn{8}{|c|}{ Tests based on global size correction } \\
\hline$\left|t\left(r_{1}\right)\right|$ & 0.00 & 0.01 & 0.00 & 5.21 & 11.98 & 1.42 & 14.83 & 16.47 \\
\hline$\left|\tilde{t}\left(r_{1}\right)\right|$ & 0.00 & 0.01 & 0.00 & 5.21 & 11.98 & 1.42 & 14.83 & 16.47 \\
\hline$\left|t\left(\hat{\rho}_{k}\right)\right|$ & 0.0 & 0.01 & 0.00 & 4.8 & & 0.97 & 12 & 14.73 \\
\hline$\left|\bar{t}\left(\hat{\rho}_{k}\right)\right|$ & 0 & 0.01 & 0.00 & 5.52 & 12.49 & 1.55 & 15.55 & 17.02 \\
\hline & \multicolumn{8}{|c|}{ Tests based on model-specific size correction } \\
\hline$\left|t\left(r_{1}\right)\right|$ & 86.38 & 90.86 & 99.78 & 19.35 & 11.98 & 31.88 & 33.28 & 33.76 \\
\hline$\left|\tilde{t}\left(r_{1}\right)\right|$ & 86.38 & 90.86 & 99.78 & 19.35 & 11.98 & 31.88 & 33.28 & 33.76 \\
\hline$\left|t\left(\hat{\rho}_{k}\right)\right|$ & 82.64 & 88.05 & 99.69 & 18.19 & 11.66 & 30.23 & 32.60 & 32.86 \\
\hline$\left|\bar{t}\left(\hat{\rho}_{k}\right)\right|$ & 85.31 & 90.28 & 99.78 & 19.50 & 12.49 & 32.03 & 33.55 & 34.07 \\
\hline
\end{tabular}


Table C.8. Empirical powers of serial dependence tests at level $\alpha=0.05$

$$
X_{t}=0.3 X_{t-1}+u_{t}, v_{t} \sim \text { Cauchy }
$$

\begin{tabular}{|c|c|c|c|c|c|c|c|c|}
\hline \multirow{2}{*}{$\begin{array}{c}\text { Sample size: } n=30 \\
\text { Test } \backslash \text { Model }\end{array}$} & \multicolumn{8}{|c|}{ Asymptotic tests and bounds } \\
\hline & M1 & M2 & M3 & M4 & M5 & M6 & M7 & M8 \\
\hline$E_{11}$ & 25.26 & 26.99 & 33.92 & 20.12 & 9.81 & 24.40 & 29.42 & 57.95 \\
\hline \multirow[t]{2}{*}{ Best bound } & 25.58 & 27.14 & 33.97 & 20.27 & 9.81 & 24.52 & 29.42 & 57.95 \\
\hline & \multicolumn{8}{|c|}{ Tests based on global size correction } \\
\hline$\left|t\left(r_{1}\right)\right|$ & 0.64 & 0.37 & 0.54 & 1.25 & 5.05 & 1.31 & 6.04 & 9.86 \\
\hline$\left|\tilde{t}\left(r_{1}\right)\right|$ & 0.64 & 0.37 & 0.54 & 1.25 & 5.05 & 1.31 & 6.04 & 9.86 \\
\hline$\left|t\left(\hat{\rho}_{k}\right)\right|$ & 0.36 & 0.17 & 0.19 & 0.85 & 4.20 & 0.71 & 2.10 & 3.31 \\
\hline$\left|\bar{t}\left(\hat{\rho}_{k}\right)\right|$ & 0.71 & 0.44 & 0.56 & 1.37 & 5.89 & 1.57 & 7.00 & 11.00 \\
\hline & \multicolumn{8}{|c|}{ Tests based on model-specific size correction } \\
\hline$\left|t\left(r_{1}\right)\right|$ & 57.56 & 59.30 & 90.60 & 27.45 & 12.55 & 23.52 & 21.68 & 23.89 \\
\hline$\left|\tilde{t}\left(r_{1}\right)\right|$ & 57.56 & 59.30 & 90.60 & 27.45 & 12.55 & 23.52 & 21.68 & 23.89 \\
\hline$\left|t\left(\hat{\rho}_{k}\right)\right|$ & 30.58 & 30.54 & 78.42 & 19.18 & 11.12 & 17.48 & 18.89 & 20.15 \\
\hline$\left|\bar{t}\left(\hat{\rho}_{k}\right)\right|$ & 51.18 & 53.48 & 88.20 & 27.06 & 13.50 & 23.34 & 22.27 & 24.29 \\
\hline Sample size: $n=60$ & \multicolumn{8}{|c|}{ Asymptotic tests and bounds } \\
\hline$E_{11}$ & 44.50 & 44.36 & 46.88 & 23.50 & 10.65 & 40.39 & 38.27 & 57.65 \\
\hline \multirow[t]{2}{*}{ Best bound } & 44.94 & 44.77 & 46.97 & 23.70 & 10.65 & 40.76 & 38.27 & 57.65 \\
\hline & \multicolumn{8}{|c|}{ Tests based on global size correction } \\
\hline$\left|t\left(r_{1}\right)\right|$ & 0.11 & 0.09 & 0.12 & 1.06 & 5.32 & 0.35 & 3.43 & 9.44 \\
\hline$\left|\tilde{t}\left(r_{1}\right)\right|$ & 0.11 & 0.09 & 0.12 & 1.06 & 5.32 & 0.35 & 3.43 & 9.44 \\
\hline$\left|t\left(\hat{\rho}_{k}\right)\right|$ & 0.10 & 0.07 & 0.08 & 0.87 & 4.83 & 0.26 & 2.43 & 7.07 \\
\hline$\left|\bar{t}\left(\hat{\rho}_{k}\right)\right|$ & 0.13 & 0.10 & 0.15 & 1.11 & 5.69 & 0.38 & 3.89 & 9.99 \\
\hline & \multicolumn{8}{|c|}{ Tests based on model-specific size correction } \\
\hline$\left|t\left(r_{1}\right)\right|$ & 88.35 & 88.79 & 96.24 & 30.78 & 13.07 & 71.52 & 20.51 & 23.76 \\
\hline$\left|\tilde{t}\left(r_{1}\right)\right|$ & 88.35 & 88.79 & 96.24 & 30.78 & 13.07 & 71.52 & 20.51 & 23.76 \\
\hline$\left|t\left(\hat{\rho}_{k}\right)\right|$ & 84.13 & 84.52 & 95.14 & 25.89 & 12.27 & 61.35 & 18.90 & 22.72 \\
\hline$\left|\bar{t}\left(\hat{\rho}_{k}\right)\right|$ & 87.18 & 87.82 & 96.13 & 31.30 & 13.75 & 69.30 & 20.54 & 24.44 \\
\hline Sample size: $n=100$ & \multicolumn{8}{|c|}{ Asymptotic tests and bounds } \\
\hline$E_{11}$ & 60.13 & 59.82 & 60.44 & 24.06 & 10.99 & 56.40 & 45.36 & 57.90 \\
\hline \multirow[t]{2}{*}{ Best bound } & 60.51 & 60.10 & 60.55 & 24.22 & 10.99 & 56.74 & 45.37 & 57.90 \\
\hline & \multicolumn{8}{|c|}{ Tests based on global size correction } \\
\hline$\left|t\left(r_{1}\right)\right|$ & 0.04 & 0.06 & 0.08 & 1.04 & 6.12 & 0.15 & 2.04 & 10.38 \\
\hline$\left|\tilde{t}\left(r_{1}\right)\right|$ & 0.04 & 0.06 & 0.08 & 1.04 & 6.12 & 0.15 & 2.04 & 10.38 \\
\hline$\left|t\left(\hat{\rho}_{k}\right)\right|$ & 0.04 & 0.05 & 0.05 & 0.91 & 5.82 & 0.13 & 1.61 & 9.14 \\
\hline$\left|\bar{t}\left(\hat{\rho}_{k}\right)\right|$ & 0.05 & 0.08 & 0.07 & 1.07 & 6.34 & 0.19 & 2.27 & 10.83 \\
\hline & \multicolumn{8}{|c|}{ Tests based on model-specific size correction } \\
\hline$\left|t\left(r_{1}\right)\right|$ & 96.59 & 96.88 & 98.14 & 31.28 & 13.72 & 92.03 & 19.23 & 24.11 \\
\hline$\left|\tilde{t}\left(r_{1}\right)\right|$ & 96.59 & 96.88 & 98.14 & 31.28 & 13.72 & 92.03 & 19.23 & 24.11 \\
\hline$\left|t\left(\hat{\rho}_{k}\right)\right|$ & 95.75 & 96.11 & 97.84 & 28.48 & 13.18 & 90.38 & 18.15 & 23.51 \\
\hline$\left|\bar{t}\left(\hat{\rho}_{k}\right)\right|$ & 96.41 & 96.71 & 98.13 & 31.50 & 14.08 & 91.49 & 19.36 & 24.49 \\
\hline
\end{tabular}


Table C.9. Empirical powers of serial dependence tests at level $\alpha=0.05$ $X_{t}=0.4 X_{t-1}+u_{t}, v_{t} \sim \mathrm{N}(0,1)$

\begin{tabular}{|c|c|c|c|c|c|c|c|c|}
\hline \multirow{2}{*}{$\begin{array}{c}\text { Sample size: } n=30 \\
\text { Test } \backslash \text { Model }\end{array}$} & \multicolumn{8}{|c|}{ Asymptotic tests and bounds } \\
\hline & M1 & M2 & M3 & M4 & M5 & M6 & M7 & M8 \\
\hline$E_{11}$ & 28.28 & 33.03 & 43.34 & 13.25 & 6.37 & 26.44 & 33.18 & 59.20 \\
\hline \multirow[t]{2}{*}{ Best bound } & 30.42 & 34.05 & 43.39 & 13.77 & 6.37 & 26.83 & 33.18 & 59.20 \\
\hline & \multicolumn{8}{|c|}{ Tests based on global size correction } \\
\hline$\left|t\left(r_{1}\right)\right|$ & 4.58 & 2.31 & 0.26 & 10.97 & 16.32 & 16.83 & 26.82 & 27.45 \\
\hline$\left|\tilde{t}\left(r_{1}\right)\right|$ & 4.58 & 2.31 & 0.26 & 10.97 & 16.32 & 16.83 & 26.82 & 27.45 \\
\hline$\left|t\left(\hat{\rho}_{k}\right)\right|$ & 2.94 & 1.14 & 0.10 & 8.23 & 14.89 & 11.03 & 21.12 & 21.72 \\
\hline$\left|\bar{t}\left(\hat{\rho}_{k}\right)\right|$ & 5.42 & 2.61 & 0.20 & 11.91 & 19.31 & 17.46 & 27.36 & 27.86 \\
\hline & \multicolumn{8}{|c|}{ Tests based on model-specific size correction } \\
\hline$\left|t\left(r_{1}\right)\right|$ & 58.70 & 79.63 & 99.65 & 29.83 & 16.32 & 37.85 & 41.41 & 42.27 \\
\hline$\left|\tilde{t}\left(r_{1}\right)\right|$ & 58.70 & 79.63 & 99.65 & 29.83 & 16.32 & 37.85 & 41.41 & 42.27 \\
\hline$\left|t\left(\hat{\rho}_{k}\right)\right|$ & 45.51 & 65.05 & 99.17 & 24.98 & 14.89 & 32.87 & 37.20 & 37.33 \\
\hline$\left|\bar{t}\left(\hat{\rho}_{k}\right)\right|$ & 54.76 & 75.52 & 99.48 & 30.57 & 19.31 & 37.81 & 40.89 & 41.41 \\
\hline Sample size: $n=60$ & \multicolumn{8}{|c|}{ Asymptotic tests and bounds } \\
\hline$E_{11}$ & 62.43 & 59.63 & 54.76 & 14.78 & 6.18 & 45.92 & 37.70 & 58.55 \\
\hline \multirow[t]{2}{*}{ Best bound } & 66.20 & 61.48 & 54.99 & 15.25 & 6.18 & 46.72 & 37.71 & 58.55 \\
\hline & \multicolumn{8}{|c|}{ Tests based on global size correction } \\
\hline$\left|t\left(r_{1}\right)\right|$ & 0.97 & 0.65 & 0.06 & 11.36 & 16.20 & 11.78 & 26.47 & 27.24 \\
\hline$\left|\tilde{t}\left(r_{1}\right)\right|$ & 0.97 & 0.65 & 0.06 & 11.36 & 16.20 & 11.78 & 26.47 & 27.24 \\
\hline$\left|t\left(\hat{\rho}_{k}\right)\right|$ & 0.70 & 0.39 & 0.03 & 10.40 & 15.86 & 9.62 & 24.17 & 25.05 \\
\hline$\left|\bar{t}\left(\hat{\rho}_{k}\right)\right|$ & 1.14 & 0.68 & 0.04 & 11.97 & 17.58 & 12.36 & 26.87 & 27.50 \\
\hline & \multicolumn{8}{|c|}{ Tests based on model-specific size correction } \\
\hline$\left|t\left(r_{1}\right)\right|$ & 86.78 & 94.00 & 99.86 & 31.25 & 16.20 & 42.53 & 41.90 & 41.42 \\
\hline$\left|\tilde{t}\left(r_{1}\right)\right|$ & 86.78 & 94.00 & 99.86 & 31.25 & 16.20 & 42.53 & 41.90 & 41.42 \\
\hline$\left|t\left(\hat{\rho}_{k}\right)\right|$ & 81.55 & 90.97 & 99.80 & 28.97 & 15.86 & 39.98 & 40.10 & 39.81 \\
\hline$\left|\bar{t}\left(\hat{\rho}_{k}\right)\right|$ & 84.86 & 92.97 & 99.88 & 32.05 & 17.58 & 42.44 & 41.86 & 41.29 \\
\hline Sample size: $n=100$ & \multicolumn{8}{|c|}{ Asymptotic tests and bounds } \\
\hline$E_{11}$ & 89.08 & 82.77 & 61.38 & 14.85 & 5.95 & 62.43 & 41.01 & 59.27 \\
\hline \multirow[t]{2}{*}{ Best bound } & 90.84 & 83.78 & 61.54 & 15.36 & 5.95 & 63.00 & 41.04 & 59.27 \\
\hline & \multicolumn{8}{|c|}{ Tests based on global size correction } \\
\hline$\left|t\left(r_{1}\right)\right|$ & 0.36 & 0.19 & 0.01 & 11.55 & 16.41 & 8.47 & 26.95 & 27.67 \\
\hline$\left|\tilde{t}\left(r_{1}\right)\right|$ & 0.36 & 0.19 & 0.01 & 11.55 & 16.41 & 8.47 & 26.95 & 27.67 \\
\hline$\left|t\left(\hat{\rho}_{k}\right)\right|$ & 0.28 & 0.11 & 0.00 & 10.76 & 16.26 & 7.07 & 25.34 & 26.37 \\
\hline$\left|\bar{t}\left(\hat{\rho}_{k}\right)\right|$ & 0.40 & 0.19 & 0.01 & 11.78 & 17.27 & 8.77 & 27.19 & 27.94 \\
\hline & \multicolumn{8}{|c|}{ Tests based on model-specific size correction } \\
\hline$\left|t\left(r_{1}\right)\right|$ & 97.89 & 99.11 & 99.98 & 31.22 & 16.41 & 49.59 & 41.41 & 41.84 \\
\hline$\left|\tilde{t}\left(r_{1}\right)\right|$ & 97.89 & 99.11 & 99.98 & 31.22 & 16.41 & 49.59 & 41.41 & 41.84 \\
\hline$\left|t\left(\hat{\rho}_{k}\right)\right|$ & 96.80 & 98.48 & 99.98 & 29.89 & 16.26 & 47.49 & 40.56 & 40.77 \\
\hline$\left|\bar{t}\left(\hat{\rho}_{k}\right)\right|$ & 97.49 & 98.85 & 99.98 & 31.57 & 17.27 & 49.36 & 41.43 & 41.98 \\
\hline
\end{tabular}


Table C.10. Empirical powers of serial dependence tests at level $\alpha=0.05$

$$
X_{t}=0.4 X_{t-1}+u_{t}, v_{t} \sim \text { Cauchy }
$$

\begin{tabular}{|c|c|c|c|c|c|c|c|c|}
\hline \multirow{2}{*}{$\begin{array}{c}\text { Sample size: } n=30 \\
\text { Test } \backslash \text { Model }\end{array}$} & \multicolumn{8}{|c|}{ Asymptotic tests and bounds } \\
\hline & M1 & M2 & M3 & M4 & M5 & M6 & M7 & M8 \\
\hline$E_{11}$ & 37.94 & 38.74 & 45.78 & 30.24 & 13.21 & 35.47 & 38.77 & 63.29 \\
\hline \multirow[t]{2}{*}{ Best bound } & 38.36 & 39.09 & 45.81 & 30.49 & 13.22 & 35.76 & 38.78 & 63.29 \\
\hline & \multicolumn{8}{|c|}{ Tests based on global size correction } \\
\hline$\left|t\left(r_{1}\right)\right|$ & 1.97 & 2.13 & 1.24 & 3.68 & 9.88 & 4.09 & 13.74 & 18.68 \\
\hline$\left|\tilde{t}\left(r_{1}\right)\right|$ & 1.97 & 2.13 & 1.24 & 3.68 & 9.88 & 4.09 & 13.74 & 18.68 \\
\hline$\left|t\left(\hat{\rho}_{k}\right)\right|$ & 1.19 & 1.18 & 0.76 & 2.38 & 8.15 & 2.62 & 9.68 & 14.56 \\
\hline$\left|\bar{t}\left(\hat{\rho}_{k}\right)\right|$ & 2.34 & 2.21 & 1.38 & 4.23 & 11.13 & 4.37 & 14.34 & 19.06 \\
\hline & \multicolumn{8}{|c|}{ Tests based on model-specific size correction } \\
\hline$\left|t\left(r_{1}\right)\right|$ & 82.35 & 84.09 & 96.07 & 67.81 & 21.05 & 61.49 & 32.19 & 33.84 \\
\hline$\left|\tilde{t}\left(r_{1}\right)\right|$ & 82.35 & 84.09 & 96.07 & 67.81 & 21.06 & 61.49 & 32.19 & 33.84 \\
\hline$\left|t\left(\hat{\rho}_{k}\right)\right|$ & 71.56 & 73.42 & 93.82 & 50.59 & 18.00 & 39.04 & 27.28 & 28.77 \\
\hline$\left|\bar{t}\left(\hat{\rho}_{k}\right)\right|$ & 79.05 & 81.06 & 95.63 & 65.15 & 22.20 & 56.77 & 32.20 & 33.77 \\
\hline Sample size: $n=60$ & \multicolumn{8}{|c|}{ Asymptotic tests and bounds } \\
\hline$E_{11}$ & 60.80 & 60.38 & 59.98 & 34.29 & 13.00 & 56.56 & 47.59 & 62.72 \\
\hline \multirow[t]{2}{*}{ Best bound } & 61.26 & 60.73 & 60.07 & 34.62 & 13.00 & 56.96 & 47.62 & 62.72 \\
\hline & \multicolumn{8}{|c|}{ Tests based on global size correction } \\
\hline$\left|t\left(r_{1}\right)\right|$ & 0.83 & 0.71 & 0.62 & 3.12 & 9.07 & 1.30 & 10.16 & 18.55 \\
\hline$\left|\tilde{t}\left(r_{1}\right)\right|$ & 0.83 & 0.71 & 0.62 & 3.12 & 9.07 & 1.30 & 10.16 & 18.55 \\
\hline$\left|t\left(\hat{\rho}_{k}\right)\right|$ & 0.63 & 0.56 & 0.54 & 2.56 & 8.57 & 1.01 & 8.60 & 16.79 \\
\hline$\left|\bar{t}\left(\hat{\rho}_{k}\right)\right|$ & 0.86 & 0.81 & 0.68 & 3.27 & 9.92 & 1.42 & 10.43 & 18.88 \\
\hline & \multicolumn{8}{|c|}{ Tests based on model-specific size correction } \\
\hline$\left|t\left(r_{1}\right)\right|$ & 96.00 & 95.99 & 98.41 & 73.40 & 19.84 & 89.86 & 32.64 & 33.35 \\
\hline$\left|\tilde{t}\left(r_{1}\right)\right|$ & 96.00 & 95.99 & 98.41 & 73.40 & 19.84 & 89.86 & 32.64 & 33.35 \\
\hline$\left|t\left(\hat{\rho}_{k}\right)\right|$ & 94.88 & 94.96 & 98.07 & 68.43 & 18.68 & 87.40 & 29.80 & 31.71 \\
\hline$\left|\bar{t}\left(\hat{\rho}_{k}\right)\right|$ & 95.88 & 95.84 & 98.32 & 73.80 & 20.70 & 89.43 & 32.80 & 33.88 \\
\hline Sample size: $n=100$ & \multicolumn{8}{|c|}{ Asymptotic tests and bounds } \\
\hline$E_{11}$ & 73.82 & 74.29 & 73.42 & 33.95 & 13.93 & 70.70 & 56.18 & 63.32 \\
\hline \multirow[t]{2}{*}{ Best bound } & 74.15 & 74.55 & 73.57 & 34.23 & 13.94 & 71.06 & 56.20 & 63.32 \\
\hline & \multicolumn{8}{|c|}{ Tests based on global size correction } \\
\hline$\left|t\left(r_{1}\right)\right|$ & 0.22 & 0.38 & 0.43 & 3.05 & 10.00 & 0.72 & 7.00 & 19.30 \\
\hline$\left|\tilde{t}\left(r_{1}\right)\right|$ & 0.22 & 0.38 & 0.43 & 3.05 & 10.00 & 0.72 & 7.00 & 19.30 \\
\hline$\left|t\left(\hat{\rho}_{k}\right)\right|$ & 0.17 & 0.28 & 0.38 & 2.61 & 9.45 & 0.60 & 6.16 & 18.15 \\
\hline$\left|\bar{t}\left(\hat{\rho}_{k}\right)\right|$ & 0.22 & 0.40 & 0.42 & 3.07 & 10.40 & 0.70 & 7.25 & 19.52 \\
\hline & \multicolumn{8}{|c|}{ Tests based on model-specific size correction } \\
\hline$\left|t\left(r_{1}\right)\right|$ & 98.64 & 98.67 & 99.20 & 72.92 & 19.93 & 97.13 & 36.37 & 33.24 \\
\hline$\left|\tilde{t}\left(r_{1}\right)\right|$ & 98.64 & 98.67 & 99.20 & 72.92 & 19.93 & 97.13 & 36.37 & 33.24 \\
\hline$\left|t\left(\hat{\rho}_{k}\right)\right|$ & 98.49 & 98.51 & 99.16 & 70.47 & 19.22 & 96.79 & 33.62 & 32.35 \\
\hline$\left|\bar{t}\left(\hat{\rho}_{k}\right)\right|$ & 98.63 & 98.64 & 99.22 & 73.30 & 20.42 & 97.08 & 36.42 & 33.53 \\
\hline
\end{tabular}


Table C.11. Empirical powers of serial dependence tests at level $\alpha=0.05$

$$
X_{t}=0.5 X_{t-1}+u_{t}, v_{t} \sim \mathrm{N}(0,1)
$$

\begin{tabular}{|c|c|c|c|c|c|c|c|c|}
\hline Sample size: $n=30$ & \multicolumn{8}{|c|}{ Asymptotic tests and bounds } \\
\hline Test $\backslash$ Model & M1 & M2 & M3 & M4 & M5 & M6 & M7 & M8 \\
\hline$E_{11}$ & 47.45 & 49.72 & 52.10 & 23.43 & 7.55 & 40.04 & 40.64 & 62.86 \\
\hline Best b & 49.99 & 50.69 & 52.19 & 24.04 & 7.55 & 40.37 & 40.64 & 62.86 \\
\hline & \multicolumn{8}{|c|}{ Tests based on global size correction } \\
\hline$\left|t\left(r_{1}\right)\right|$ & 14.43 & 9.77 & 1.17 & 20.40 & 21.54 & 29.70 & 37.74 & 37.76 \\
\hline$\left|\tilde{t}\left(r_{1}\right)\right|$ & 14.43 & 9.77 & 1.17 & & & & 37.74 & 37.76 \\
\hline$\left|t\left(\hat{\rho}_{k}\right)\right|$ & 9.42 & 5.12 & 0.60 & & 20.62 & & 32.34 & 32.41 \\
\hline$\left|\bar{t}\left(\hat{\rho}_{k}\right)\right|$ & 14.81 & 9.45 & 1.37 & 21.68 & 24.96 & 29.29 & 37.27 & 37.30 \\
\hline & \multicolumn{8}{|c|}{ Tests based on model-specific size correction } \\
\hline$\left|t\left(r_{1}\right)\right|$ & 77.31 & 91.97 & 99.90 & 44.65 & 21.54 & 49.11 & 50.63 & 49.97 \\
\hline$\left|\tilde{t}\left(r_{1}\right)\right|$ & 7.31 & 91.97 & 99.90 & 44 & 21.54 & & 50 & 49.97 \\
\hline$\left|t\left(\hat{\rho}_{k}\right)\right|$ & 5.24 & 84.46 & 99.70 & 38 & 20.62 & & 45.84 & 44.93 \\
\hline$\left|\bar{t}\left(\hat{\rho}_{k}\right)\right|$ & 2.53 & 89.49 & 99.82 & 45.17 & 24.96 & 48.20 & 49.47 & 48.67 \\
\hline Sample size: $n$ & \multicolumn{8}{|c|}{ Asymptotic tests and bounds } \\
\hline$E_{11}$ & 67 & 79.70 & 61.49 & 24.03 & 7.75 & 61.55 & 44.23 & 62.83 \\
\hline Best b & 8.47 & 80.68 & 61.55 & 24.58 & 7.75 & 62.00 & & 62.83 \\
\hline & \multicolumn{8}{|c|}{ Tests based on global size correction } \\
\hline$t\left(r_{1}\right.$ & 8.93 & 6.55 & 0.85 & 20.34 & 22.13 & 26.52 & 37.75 & 37.71 \\
\hline$\left|\tilde{t}\left(r_{1}\right)\right|$ & 8.93 & 6.55 & 0.85 & & & & 37.75 & 37.71 \\
\hline$\left|t\left(\hat{\rho}_{k}\right)\right|$ & 6.64 & 4.49 & 0.59 & & 21 & & 35 & 35.33 \\
\hline$\left|\bar{t}\left(\hat{\rho}_{k}\right)\right|$ & 9.15 & 6.66 & 0.89 & 21.0 & 24.18 & 26.49 & 37.62 & 37.54 \\
\hline & \multicolumn{8}{|c|}{ Tests based on model-specific size correction } \\
\hline$\left|t\left(r_{1}\right)\right|$ & 97.03 & 98.91 & 100 & 45.48 & 22.13 & 57.79 & 50.01 & 50.07 \\
\hline$\left|\tilde{t}\left(r_{1}\right)\right|$ & 7.03 & 98.91 & 10 & 45 & 22 & 57 & 50 & 50.07 \\
\hline$t\left(\hat{\rho}_{k}\right)$ & 517 & 98.08 & 99.99 & 42 & 21 & & 48 & 48.14 \\
\hline $\bar{t}\left(\hat{\rho}_{k}\right.$ & 96.38 & 98.58 & 100 & 46. & & & & 49.66 \\
\hline Sample size: $n=100$ & \multicolumn{8}{|c|}{ Asymptotic tests and bounds } \\
\hline$E_{11}$ & 8.61 & 94.48 & 68.57 & 23.31 & 6.89 & 74.57 & 47.94 & 62.67 \\
\hline Best bound & 0907 & 94.78 & 68.59 & 23.92 & 6.89 & 75.03 & 47 & 62.67 \\
\hline & \multicolumn{8}{|c|}{ Tests based on global size correction } \\
\hline$\left|t\left(r_{1}\right)\right|$ & 5.73 & 4.19 & 0.52 & 19.73 & 21.03 & 23.61 & 38.39 & 37.62 \\
\hline$\left|\tilde{t}\left(r_{1}\right)\right|$ & 5.73 & 4.19 & 0.52 & & 21.03 & & 38.39 & 37.62 \\
\hline$\left|t\left(\hat{\rho}_{k}\right)\right|$ & 4.32 & 3.28 & 0.3 & & & & & 36.22 \\
\hline$\left|\bar{t}\left(\hat{\rho}_{k}\right)\right|$ & & 4.54 & 0.58 & 20.25 & 22.22 & 23.59 & 38.38 & 37.61 \\
\hline & \multicolumn{8}{|c|}{ Tests based on model-specific size correction } \\
\hline$\left|t\left(r_{1}\right)\right|$ & 9.85 & 99.98 & 100 & 43.91 & 21.03 & 66.96 & 50.96 & 49.84 \\
\hline$\left|\tilde{t}\left(r_{1}\right)\right|$ & & 99.98 & 100 & 43.91 & 21 & & 50.96 & 49.84 \\
\hline$\left|t\left(\hat{\rho}_{k}\right)\right|$ & 99.76 & 99.93 & 100 & 42.76 & 20.85 & 64.92 & 50.03 & 48.76 \\
\hline$\left|\bar{t}\left(\hat{\rho}_{k}\right)\right|$ & 99.84 & 99.96 & 100 & 44.51 & 22.22 & 66.76 & 50.83 & 49.79 \\
\hline
\end{tabular}


Table C.12. Empirical powers of serial dependence tests at level $\alpha=0.05$

$$
X_{t}=0.5 X_{t-1}+u_{t}, v_{t} \sim \text { Cauchy }
$$

\begin{tabular}{|c|c|c|c|c|c|c|c|c|}
\hline \multirow{2}{*}{$\begin{array}{c}\text { Sample size: } n=30 \\
\text { Test } \backslash \text { Model }\end{array}$} & \multicolumn{8}{|c|}{ Asymptotic tests and bounds } \\
\hline & M1 & M2 & M3 & M4 & M5 & M6 & M7 & M8 \\
\hline$E_{11}$ & 51.25 & 52.16 & 55.57 & 41.39 & 17.34 & 48.53 & 47.78 & 67.07 \\
\hline \multirow[t]{2}{*}{ Best bound } & 51.94 & 52.53 & 55.59 & 41.73 & 17.34 & 48.92 & 47.79 & 67.07 \\
\hline & \multicolumn{8}{|c|}{ Tests based on global size correction } \\
\hline$\left|t\left(r_{1}\right)\right|$ & 7.96 & 7.38 & 3.84 & 10.30 & 15.27 & 11.19 & 25.16 & 29.71 \\
\hline$\left|\tilde{t}\left(r_{1}\right)\right|$ & 7.96 & 7.38 & 3.84 & 10.30 & 15.27 & 11.19 & 25.16 & 29.71 \\
\hline$\left|t\left(\hat{\rho}_{k}\right)\right|$ & 4.60 & 4.20 & 2.11 & 6.90 & 13.09 & 7.24 & 19.13 & 24.03 \\
\hline$\left|\bar{t}\left(\hat{\rho}_{k}\right)\right|$ & 7.91 & 7.58 & 3.77 & 10.93 & 17.55 & 11.46 & 24.56 & 29.21 \\
\hline & \multicolumn{8}{|c|}{ Tests based on model-specific size correction } \\
\hline$\left|t\left(r_{1}\right)\right|$ & 91.78 & 92.38 & 97.67 & 83.93 & 31.34 & 81.70 & 56.40 & 51.77 \\
\hline$\left|\tilde{t}\left(r_{1}\right)\right|$ & 91.78 & 92.38 & 97.67 & 83.93 & 31.34 & 81.70 & 56.40 & 51.77 \\
\hline$\left|t\left(\hat{\rho}_{k}\right)\right|$ & 86.69 & 88.14 & 96.47 & 77.02 & 27.15 & 73.37 & 43.55 & 41.55 \\
\hline$\left|\bar{t}\left(\hat{\rho}_{k}\right)\right|$ & 90.00 & 91.37 & 97.28 & 82.91 & 33.15 & 79.37 & 54.26 & 49.15 \\
\hline Sample size: $n=60$ & \multicolumn{8}{|c|}{ Asymptotic tests and bounds } \\
\hline$E_{11}$ & 73.68 & 73.52 & 72.35 & 46.78 & 16.66 & 69.72 & 56.22 & 66.08 \\
\hline \multirow[t]{2}{*}{ Best bound } & 74.14 & 73.86 & 72.49 & 47.08 & 16.67 & 70.09 & 56.23 & 66.08 \\
\hline & \multicolumn{8}{|c|}{ Tests based on global size correction } \\
\hline$\left|t\left(r_{1}\right)\right|$ & 4.20 & 4.24 & 2.57 & 9.21 & 15.12 & 5.78 & 20.34 & 28.67 \\
\hline$\left|\tilde{t}\left(r_{1}\right)\right|$ & 4.20 & 4.24 & 2.57 & 9.21 & 15.12 & 5.78 & 20.34 & 28.67 \\
\hline$\left|t\left(\hat{\rho}_{k}\right)\right|$ & 3.20 & 2.98 & 1.89 & 7.49 & 14.40 & 4.36 & 17.70 & 26.48 \\
\hline$\left|\bar{t}\left(\hat{\rho}_{k}\right)\right|$ & 4.29 & 4.09 & 2.46 & 9.58 & 16.25 & 5.92 & 19.99 & 28.54 \\
\hline & \multicolumn{8}{|c|}{ Tests based on model-specific size correction } \\
\hline$\left|t\left(r_{1}\right)\right|$ & 97.97 & 98.07 & 98.98 & 87.69 & 31.44 & 95.34 & 62.21 & 50.68 \\
\hline$\left|\tilde{t}\left(r_{1}\right)\right|$ & 97.97 & 98.07 & 98.98 & 87.69 & 31.44 & 95.34 & 62.21 & 50.68 \\
\hline$\left|t\left(\hat{\rho}_{k}\right)\right|$ & 97.55 & 97.67 & 98.85 & 85.69 & 29.42 & 94.27 & 57.72 & 46.17 \\
\hline$\left|\bar{t}\left(\hat{\rho}_{k}\right)\right|$ & 97.87 & 97.93 & 98.93 & 87.71 & 32.73 & 95.04 & 61.67 & 51.15 \\
\hline Sample size: $n=100$ & \multicolumn{8}{|c|}{ Asymptotic tests and bounds } \\
\hline$E_{11}$ & 85.82 & 85.87 & 83.77 & 46.20 & 17.46 & 82.89 & 65.42 & 66.46 \\
\hline \multirow[t]{2}{*}{ Best bound } & 86.05 & 86.03 & 83.84 & 46.60 & 17.48 & 83.17 & 65.44 & 66.46 \\
\hline & \multicolumn{8}{|c|}{ Tests based on global size correction } \\
\hline$\left|t\left(r_{1}\right)\right|$ & 2.22 & 2.54 & 1.99 & 9.61 & 16.14 & 3.53 & 16.67 & 29.31 \\
\hline$\left|\tilde{t}\left(r_{1}\right)\right|$ & 2.22 & 2.54 & 1.99 & 9.61 & 16.14 & 3.53 & 16.67 & 29.31 \\
\hline$\left|t\left(\hat{\rho}_{k}\right)\right|$ & 1.69 & 2.02 & 1.63 & 8.58 & 15.67 & 2.94 & 15.29 & 27.88 \\
\hline$\left|\bar{t}\left(\hat{\rho}_{k}\right)\right|$ & 2.20 & 2.55 & 2.05 & 9.69 & 16.90 & 3.63 & 16.74 & 29.26 \\
\hline & \multicolumn{8}{|c|}{ Tests based on model-specific size correction } \\
\hline$\left|t\left(r_{1}\right)\right|$ & 99.30 & 99.28 & 99.49 & 87.88 & 31.58 & 98.93 & 72.71 & 51.19 \\
\hline$\left|\tilde{t}\left(r_{1}\right)\right|$ & 99.30 & 99.28 & 99.49 & 87.88 & 31.58 & 98.93 & 72.71 & 51.19 \\
\hline$\left|t\left(\hat{\rho}_{k}\right)\right|$ & 99.23 & 99.28 & 99.48 & 87.04 & 30.11 & 98.84 & 70.69 & 48.27 \\
\hline$\left|\bar{t}\left(\hat{\rho}_{k}\right)\right|$ & 99.28 & 99.32 & 99.50 & 88.07 & 32.59 & 98.91 & 72.38 & 51.38 \\
\hline
\end{tabular}


Table C.13. Empirical powers of serial dependence tests at level $\alpha=0.05$

$$
X_{t}=0.6 X_{t-1}+u_{t}, v_{t} \sim \mathrm{N}(0,1)
$$

\begin{tabular}{|c|c|c|c|c|c|c|c|c|}
\hline Sample size: $n=30$ & \multicolumn{8}{|c|}{ Asymptotic tests and bounds } \\
\hline Test $\backslash$ Model & M1 & M2 & M3 & M4 & M5 & M6 & M7 & M8 \\
\hline$E_{11}$ & 68.96 & 65.56 & 63.09 & 34.95 & 9.97 & 53.71 & 52.46 & 67.11 \\
\hline Best 1 & 0.94 & 66.68 & 63.18 & 35.83 & 9.97 & 54.10 & 52.46 & 67.11 \\
\hline & \multicolumn{8}{|c|}{ Tests based on global size correction } \\
\hline$\left|t\left(r_{1}\right)\right|$ & 4.35 & 35.81 & 15.94 & 32.37 & 27.19 & 45.33 & 47.90 & 49.10 \\
\hline$\left|\tilde{t}\left(r_{1}\right)\right|$ & 34.35 & 35.81 & 15.94 & & 27.19 & 45.33 & 47.90 & 49.10 \\
\hline & 3.89 & 19.78 & 3.5 & 27.24 & 26.47 & 37.12 & 42.54 & 43.53 \\
\hline $\bar{t}\left(\hat{\rho}_{k}\right.$ & 31.64 & 31.12 & 8.87 & 34.05 & 31.69 & 43.08 & 46.50 & 47.49 \\
\hline & \multicolumn{8}{|c|}{ Tests based on model-specific size correction } \\
\hline$\left|t\left(r_{1}\right)\right|$ & 90.29 & 97.53 & 99.94 & 58.84 & 27.19 & 62.20 & 58.44 & 59.99 \\
\hline $\mid \tilde{t}\left(r_{1}\right.$ & .29 & 97.53 & 99.94 & 58.84 & 27.19 & 62.20 & 58.44 & 59.99 \\
\hline$\left|t\left(\hat{\rho}_{k}\right)\right|$ & 1.31 & 93.74 & 99.88 & 52.13 & 26.47 & 56.07 & 53.92 & 55.11 \\
\hline$\left|\bar{t}\left(\hat{\rho}_{k}\right)\right|$ & 86.29 & 96.07 & 99.91 & 58.12 & 31.69 & 60.24 & 56.90 & 58.30 \\
\hline Sample size: $n=60$ & \multicolumn{8}{|c|}{ Asymptotic tests and bounds } \\
\hline$E_{1}$ & .88 & 90.38 & 71.61 & 35.89 & 9.98 & 72.50 & 55.79 & 65.55 \\
\hline Best b & 9 & 90.82 & 71.67 & 36.49 & 9.98 & 72.94 & 55.79 & 65.55 \\
\hline & \multicolumn{8}{|c|}{ Tests based on global size correction } \\
\hline$t(r$ & 4.58 & 36.63 & 14.70 & 32.10 & 26.72 & 44.53 & 48.28 & 47.66 \\
\hline$\left|\tilde{t}\left(r_{1}\right)\right|$ & .58 & 36.63 & 14.70 & & 26 & 44.53 & 48 & 47.66 \\
\hline$\left|t\left(\hat{\rho}_{k}\right)\right|$ & 7.68 & 27.27 & 6.41 & & 26 & 40.52 & 45 & 45.40 \\
\hline$\left|\bar{t}\left(\hat{\rho}_{k}\right)\right|$ & 3 & 33.96 & 11.70 & 33.29 & 29.16 & 43.71 & 47.65 & 47.09 \\
\hline & \multicolumn{8}{|c|}{ Tests based on model-specific size correction } \\
\hline$\left|t\left(r_{1}\right)\right|$ & $.4 \mathrm{c}$ & 99.85 & 10 & 59.22 & 26.72 & 71.72 & 59.17 & 57.41 \\
\hline$\left|\tilde{t}\left(r_{1}\right)\right|$ & 49 & 99.85 & 10 & 5 & 26.72 & 71.72 & 59.17 & 57 \\
\hline$\left|t\left(\hat{\rho}_{k}\right)\right|$ & c & 99.63 & 100 & 56.57 & 26.69 & 68.53 & 57.50 & 55.57 \\
\hline$\left|\bar{t}\left(\hat{\rho}_{k}\right)\right|$ & 99.30 & 99.73 & 100 & 59.89 & 29.16 & 70.96 & 58.71 & 56.92 \\
\hline Sample size: $n=10$ & \multicolumn{8}{|c|}{ Asymptotic tests and bounds } \\
\hline$E_{11}$ & 99.8 & 98.25 & 79.71 & & 10.07 & 83.82 & 59.75 & 66.08 \\
\hline Best b & , & 98.32 & 79.71 & 37.22 & 10.07 & 84.02 & 59.76 & 66 \\
\hline & \multicolumn{8}{|c|}{ Tests based on global size correction } \\
\hline$\left|t\left(r_{1}\right)\right|$ & 36.66 & 37.29 & 18.13 & 33.75 & 27.72 & 43.99 & 48.78 & 48.41 \\
\hline$\left|\tilde{t}\left(r_{1}\right)\right|$ & & 37.29 & 18.13 & & 27.72 & 43.99 & 48.78 & 48.41 \\
\hline & & 30.19 & 9.4 & & & 40.94 & & 47.09 \\
\hline$\left|\bar{t}\left(\hat{\rho}_{k}\right)\right|$ & 35.47 & 35.65 & 15.61 & 34.33 & 29.14 & 43.61 & 48.54 & 48.18 \\
\hline & \multicolumn{8}{|c|}{ Tests based on model-specific size correction } \\
\hline$\left|t\left(r_{1}\right)\right|$ & 99 & 100 & 100 & 59.60 & 27.72 & 81.23 & 59.38 & 58.51 \\
\hline$\left|\tilde{t}\left(r_{1}\right)\right|$ & & 100 & 100 & 59.60 & 27.72 & 81.23 & 59.38 & 58.51 \\
\hline$\left|t\left(\hat{\rho}_{k}\right)\right|$ & 99. & 100 & 100 & & 27.48 & 79.38 & 58.48 & 57.61 \\
\hline$\left|\bar{t}\left(\hat{\rho}_{k}\right)\right|$ & 100 & 100 & 100 & 59.88 & 29.14 & 80.56 & 59.19 & 58.36 \\
\hline
\end{tabular}


Table C.14. Empirical powers of serial dependence tests at level $\alpha=0.05$

$$
X_{t}=0.6 X_{t-1}+u_{t}, v_{t} \sim \text { Cauchy }
$$

\begin{tabular}{|c|c|c|c|c|c|c|c|c|}
\hline \multirow{2}{*}{$\begin{array}{c}\text { Sample size: } n=30 \\
\text { Test } \backslash \text { Model }\end{array}$} & \multicolumn{8}{|c|}{ Asymptotic tests and bounds } \\
\hline & M1 & M2 & M3 & M4 & M5 & M6 & M7 & M8 \\
\hline$E_{11}$ & 64.71 & 65.24 & 68.09 & 52.83 & 20.39 & 60.79 & 56.48 & 69.20 \\
\hline Best bound & 65.25 & 65.70 & 68.16 & 53.30 & 20.40 & 61.21 & 56.51 & 69.20 \\
\hline & \multicolumn{8}{|c|}{ Tests based on global size correction } \\
\hline$\left|t\left(r_{1}\right)\right|$ & 29.18 & 29.56 & 23.06 & 29.25 & 25.02 & 32.92 & 40.97 & 44.78 \\
\hline$\left|\tilde{t}\left(r_{1}\right)\right|$ & 29.18 & 29.56 & 23.06 & 29.25 & 25.02 & 32.92 & 40.97 & 44.78 \\
\hline$\left|t\left(\hat{\rho}_{k}\right)\right|$ & 15.63 & 15.66 & 9.28 & 18.06 & 21.77 & 19.74 & 30.35 & 34.77 \\
\hline$\left|\bar{t}\left(\hat{\rho}_{k}\right)\right|$ & 26.33 & 26.62 & 19.05 & 28.52 & 28.45 & 29.51 & 37.92 & 41.80 \\
\hline & \multicolumn{8}{|c|}{ Tests based on model-specific size correction } \\
\hline$\left|t\left(r_{1}\right)\right|$ & 95.33 & 96.10 & 98.66 & 92.23 & 48.31 & 89.78 & 70.89 & 66.96 \\
\hline$\left|\tilde{t}\left(r_{1}\right)\right|$ & 95.33 & 96.10 & 98.66 & 92.23 & 48.31 & 89.78 & 70.89 & 66.96 \\
\hline$\left|t\left(\hat{\rho}_{k}\right)\right|$ & 92.90 & 93.99 & 98.01 & 88.80 & 45.11 & 85.26 & 64.61 & 59.94 \\
\hline$\left|\bar{t}\left(\hat{\rho}_{k}\right)\right|$ & 94.47 & 95.40 & 98.41 & 91.48 & 51.80 & 88.07 & 69.25 & 65.23 \\
\hline Sample size: $n=60$ & \multicolumn{8}{|c|}{ Asymptotic tests and bounds } \\
\hline$E_{11}$ & 84.86 & 85.17 & 84.22 & 58.25 & 20.66 & 81.90 & 67.32 & 69.78 \\
\hline \multirow[t]{2}{*}{ Best bound } & 85.24 & 85.45 & 84.30 & 58.62 & 20.66 & 82.16 & 67.38 & 69.78 \\
\hline & \multicolumn{8}{|c|}{ Tests based on global size correction } \\
\hline$\left|t\left(r_{1}\right)\right|$ & 27.47 & 26.67 & 22.23 & 29.03 & 24.92 & 28.56 & 39.40 & 44.25 \\
\hline$\left|\tilde{t}\left(r_{1}\right)\right|$ & 27.47 & 26.67 & 22.23 & 29.03 & 24.92 & 28.56 & 39.40 & 44.25 \\
\hline$\left|t\left(\hat{\rho}_{k}\right)\right|$ & 19.02 & 18.43 & 13.72 & 23.45 & 23.51 & 20.48 & 33.38 & 39.45 \\
\hline$\left|\bar{t}\left(\hat{\rho}_{k}\right)\right|$ & 26.09 & 25.44 & 21.10 & 28.56 & 26.65 & 26.90 & 37.85 & 42.98 \\
\hline & \multicolumn{8}{|c|}{ Tests based on model-specific size correction } \\
\hline$\left|t\left(r_{1}\right)\right|$ & 98.75 & 98.87 & 99.53 & 93.90 & 48.95 & 98.02 & 77.64 & 66.68 \\
\hline$\left|\tilde{t}\left(r_{1}\right)\right|$ & 98.75 & 98.87 & 99.53 & 93.90 & 48.95 & 98.02 & 77.64 & 66.68 \\
\hline$\left|t\left(\hat{\rho}_{k}\right)\right|$ & 98.58 & 98.73 & 99.44 & 92.93 & 47.43 & 97.62 & 75.26 & 64.55 \\
\hline$\left|\bar{t}\left(\hat{\rho}_{k}\right)\right|$ & 98.72 & 98.87 & 99.48 & 93.92 & 50.63 & 97.85 & 76.98 & 66.36 \\
\hline Sample size: $n=100$ & \multicolumn{8}{|c|}{ Asymptotic tests and bounds } \\
\hline$E_{11}$ & 92.35 & 92.16 & 91.36 & 59.45 & 20.79 & 91.19 & 74.42 & 69.69 \\
\hline \multirow[t]{2}{*}{ Best bound } & 92.40 & 92.27 & 91.45 & 59.91 & 20.80 & 91.31 & 74.46 & 69.69 \\
\hline & \multicolumn{8}{|c|}{ Tests based on global size correction } \\
\hline$\left|t\left(r_{1}\right)\right|$ & 26.81 & 24.84 & 21.84 & 29.96 & 25.14 & 28.14 & 38.51 & 45.23 \\
\hline$\left|\tilde{t}\left(r_{1}\right)\right|$ & 26.81 & 24.84 & 21.84 & 29.96 & 25.14 & 28.14 & 38.51 & 45.23 \\
\hline$\left|t\left(\hat{\rho}_{k}\right)\right|$ & 19.99 & 18.18 & 15.44 & 26.20 & 24.27 & 21.29 & 33.81 & 42.56 \\
\hline$\left|\bar{t}\left(\hat{\rho}_{k}\right)\right|$ & 25.99 & 24.10 & 21.91 & 30.05 & 26.21 & 27.27 & 37.87 & 44.66 \\
\hline & \multicolumn{8}{|c|}{ Tests based on model-specific size correction } \\
\hline$\left|t\left(r_{1}\right)\right|$ & 99.40 & 99.55 & 99.77 & 94.00 & 47.37 & 99.37 & 84.44 & 66.89 \\
\hline$\left|\tilde{t}\left(r_{1}\right)\right|$ & 99.40 & 99.55 & 99.77 & 94.00 & 47.37 & 99.37 & 84.44 & 66.89 \\
\hline$\left|t\left(\hat{\rho}_{k}\right)\right|$ & 99.37 & 99.53 & 99.71 & 93.61 & 46.48 & 99.32 & 83.35 & 65.70 \\
\hline$\left|\bar{t}\left(\hat{\rho}_{k}\right)\right|$ & 99.39 & 99.54 & 99.73 & 94.03 & 48.63 & 99.34 & 84.11 & 66.73 \\
\hline
\end{tabular}


Table C.15. Empirical powers of serial dependence tests at level $\alpha=0.05$

$$
X_{t}=0.8 X_{t-1}+u_{t}, v_{t} \sim \mathrm{N}(0,1)
$$

\begin{tabular}{|c|c|c|c|c|c|c|c|c|}
\hline \multirow{2}{*}{$\begin{array}{c}\text { Sample size: } n=30 \\
\text { Test } \backslash \text { Model }\end{array}$} & \multicolumn{8}{|c|}{ Asymptotic tests and bounds } \\
\hline & M1 & M2 & M3 & M4 & M5 & M6 & M7 & M8 \\
\hline$E_{11}$ & 93.90 & 91.48 & 97.90 & 65.82 & 15.77 & 78.10 & 72.23 & 74.13 \\
\hline \multirow[t]{2}{*}{ Best bound } & 94.47 & 91.91 & 98.00 & 66.59 & 15.77 & 78.50 & 72.25 & 74.13 \\
\hline & \multicolumn{8}{|c|}{ Tests based on global size correction } \\
\hline$\left|t\left(r_{1}\right)\right|$ & 79.81 & 88.93 & 98.92 & 63.50 & 36.01 & 72.90 & 68.82 & 68.96 \\
\hline$\left|\tilde{t}\left(r_{1}\right)\right|$ & 79.81 & 88.93 & 98.92 & 63.50 & 36.01 & 72.90 & 68.82 & 68.96 \\
\hline$\left|t\left(\hat{\rho}_{k}\right)\right|$ & 64.35 & 76.47 & 97.02 & 56.26 & 35.98 & 63.11 & 61.58 & 61.49 \\
\hline$\left|\bar{t}\left(\hat{\rho}_{k}\right)\right|$ & 71.63 & 83.00 & 97.99 & 63.41 & 42.17 & 67.29 & 64.34 & 64.40 \\
\hline & \multicolumn{8}{|c|}{ Tests based on model-specific size correction } \\
\hline$\left|t\left(r_{1}\right)\right|$ & 98.82 & 99.73 & 100 & 83.71 & 36.01 & 83.08 & 75.45 & 75.22 \\
\hline$\left|\tilde{t}\left(r_{1}\right)\right|$ & 98.82 & 99.73 & 100 & 83.71 & 36.01 & 83.08 & 75.45 & 75.22 \\
\hline$\left|t\left(\hat{\rho}_{k}\right)\right|$ & 95.95 & 98.79 & 100 & 78.45 & 35.98 & 76.54 & 69.44 & 69.40 \\
\hline$\left|\bar{t}\left(\hat{\rho}_{k}\right)\right|$ & 97.27 & 99.25 & 100 & 82.67 & 42.17 & 79.26 & 71.64 & 71.35 \\
\hline Sample size: $n=60$ & \multicolumn{8}{|c|}{ Asymptotic tests and bounds } \\
\hline$E_{11}$ & 99.97 & 99.49 & 99.78 & 67.16 & 14.80 & 90.96 & 72.70 & 74.84 \\
\hline \multirow[t]{2}{*}{ Best bound } & 99.97 & 99.51 & 99.79 & 67.95 & 14.80 & 91.29 & 72.74 & 74.84 \\
\hline & \multicolumn{8}{|c|}{ Tests based on global size correction } \\
\hline$\left|t\left(r_{1}\right)\right|$ & 93.83 & 96.38 & 99.45 & 63.15 & 35.18 & 78.99 & 68.75 & 69.07 \\
\hline$\left|\tilde{t}\left(r_{1}\right)\right|$ & 93.83 & 96.38 & 99.45 & 63.15 & 35.18 & 78.99 & 68.75 & 69.07 \\
\hline$\left|t\left(\hat{\rho}_{k}\right)\right|$ & 88.33 & 92.24 & 99.03 & 60.75 & 35.56 & 73.99 & 65.63 & 65.90 \\
\hline$\left|\bar{t}\left(\hat{\rho}_{k}\right)\right|$ & 90.58 & 93.91 & 99.21 & 63.81 & 38.33 & 75.83 & 66.80 & 67.04 \\
\hline & \multicolumn{8}{|c|}{ Tests based on model-specific size correction } \\
\hline$\left|t\left(r_{1}\right)\right|$ & 99.99 & 99.99 & 100 & 84.07 & 35.18 & 92.32 & 75.48 & 75.77 \\
\hline$\left|\tilde{t}\left(r_{1}\right)\right|$ & 99.99 & 99.99 & 100 & 84.07 & 35.18 & 92.32 & 75.48 & 75.77 \\
\hline$\left|t\left(\hat{\rho}_{k}\right)\right|$ & 99.99 & 99.99 & 100 & 82.18 & 35.56 & 89.51 & 73.26 & 73.22 \\
\hline$\left|\bar{t}\left(\hat{\rho}_{k}\right)\right|$ & 99.99 & 99.99 & 100 & 84.30 & 38.33 & 90.52 & 74.01 & 74.05 \\
\hline Sample size: $n=100$ & \multicolumn{8}{|c|}{ Asymptotic tests and bounds } \\
\hline$E_{11}$ & 100 & 100 & 99.99 & 68.50 & 15.78 & 96.78 & 74.58 & 74.56 \\
\hline \multirow[t]{2}{*}{ Best bound } & 100 & 100 & 99.99 & 69.29 & 15.78 & 96.91 & 74.62 & 74.56 \\
\hline & \multicolumn{8}{|c|}{ Tests based on global size correction } \\
\hline$\left|t\left(r_{1}\right)\right|$ & 98.26 & 98.87 & 99.92 & 65.44 & 36.47 & 85.11 & 69.48 & 68.82 \\
\hline$\left|\tilde{t}\left(r_{1}\right)\right|$ & 98.26 & 98.87 & 99.92 & 65.44 & 36.47 & 85.11 & 69.48 & 68.82 \\
\hline$\left|t\left(\hat{\rho}_{k}\right)\right|$ & 96.71 & 97.75 & 99.72 & 63.99 & 36.55 & 81.96 & 67.80 & 67.16 \\
\hline$\left|\bar{t}\left(\hat{\rho}_{k}\right)\right|$ & 97.51 & 98.27 & 99.80 & 65.95 & 38.45 & 83.39 & 68.48 & 67.92 \\
\hline & \multicolumn{8}{|c|}{ Tests based on model-specific size correction } \\
\hline$\left|t\left(r_{1}\right)\right|$ & 100 & 100 & 100 & 84.38 & 36.47 & 96.91 & 76.23 & 75.45 \\
\hline$\left|\tilde{t}\left(r_{1}\right)\right|$ & 100 & 100 & 100 & 84.38 & 36.47 & 96.91 & 76.23 & 75.45 \\
\hline$\left|t\left(\hat{\rho}_{k}\right)\right|$ & 100 & 100 & 100 & 83.55 & 36.55 & 96.09 & 74.82 & 74.12 \\
\hline$\left|\bar{t}\left(\hat{\rho}_{k}\right)\right|$ & 100 & 100 & 100 & 84.72 & 38.45 & 96.49 & 75.39 & 74.57 \\
\hline
\end{tabular}


Table C.16. Empirical powers of serial dependence tests at level $\alpha=0.05$

$$
X_{t}=0.8 X_{t-1}+u_{t}, v_{t} \sim \text { Cauchy }
$$

\begin{tabular}{|c|c|c|c|c|c|c|c|c|}
\hline Sample size: $n=30$ & \multicolumn{8}{|c|}{ Asymptotic tests and bounds } \\
\hline Test $\backslash$ Model & M1 & M2 & M3 & M4 & M5 & M6 & M7 & M8 \\
\hline$E_{11}$ & 88.30 & 88.95 & 92.03 & 76.05 & 30.26 & 86.24 & 79.00 & 79.59 \\
\hline Best & 3.72 & 89.40 & 92.31 & 76.58 & 30.32 & 86.75 & 79.21 & 79.59 \\
\hline & \multicolumn{8}{|c|}{ Tests based on global size correction } \\
\hline$\left|t\left(r_{1}\right)\right|$ & 86.57 & 88.41 & 94.35 & 80.57 & 50.15 & 86.21 & 80.41 & 78.14 \\
\hline$\left|\tilde{t}\left(r_{1}\right)\right|$ & 86.57 & 88.41 & 94.35 & 80.57 & & & & 78.14 \\
\hline & 5.95 & 78.92 & 88.99 & 73.43 & & 76.25 & 12.00 & 70.58 \\
\hline $\mid \bar{t}(\hat{r}$ & 2.05 & 84.24 & 92.33 & 79.38 & 55.30 & 81.56 & 76.27 & 73.82 \\
\hline & \multicolumn{8}{|c|}{ Tests based on model-specific size correction } \\
\hline$\left|t\left(r_{1}\right)\right|$ & 18 & 98.57 & 99.42 & 97.82 & 66.52 & 97.13 & 87.71 & 84.01 \\
\hline$\left|\tilde{t}\left(r_{1}\right)\right|$ & 3.18 & 98.57 & 99.42 & 97.82 & & 97 & & 84.01 \\
\hline$\left|t\left(\hat{\rho}_{k}\right)\right|$ & 97.25 & 97.92 & 99.16 & 96.70 & & 95.28 & 83 & 79.24 \\
\hline$\left|\bar{t}\left(\hat{\rho}_{k}\right)\right|$ & 97.66 & 98.30 & 99.28 & 97.54 & 72.07 & 96.10 & 85.24 & 80.98 \\
\hline Sample size: $n=60$ & \multicolumn{8}{|c|}{ Asymptotic tests and bounds } \\
\hline$E_{11}$ & 97.34 & 97.63 & 97.46 & 79.97 & 28.28 & 96.62 & 87.05 & 79.30 \\
\hline Best bc & 2 & 97.73 & 97.56 & 80.28 & 28.31 & 96.73 & 87.30 & 79.30 \\
\hline & \multicolumn{8}{|c|}{ Tests based on global size correction } \\
\hline$\left|t\left(r_{1}\right)\right|$ & 94.30 & 95.24 & 97.01 & 82.39 & 47.60 & 93.87 & 85.2 & 76.82 \\
\hline$\left|\tilde{t}\left(r_{1}\right)\right|$ & 94.30 & 95.24 & 97.01 & 82.39 & & & & 76.82 \\
\hline$\left|t\left(\hat{\rho}_{k}\right)\right|$ & 91.94 & 93.25 & 95.87 & 80.33 & & 91 & & 73.97 \\
\hline$\left|\bar{t}\left(\hat{\rho}_{k}\right)\right|$ & 3.12 & 94.55 & 96.52 & 82.61 & 50.45 & 92.47 & 83.36 & 75.01 \\
\hline & \multicolumn{8}{|c|}{ Tests based on model-specific size correction } \\
\hline$\left|t\left(r_{1}\right)\right|$ & 99.47 & 99.52 & 99.84 & 98.45 & 65.07 & 99.21 & 92.54 & 82.80 \\
\hline$\left|\tilde{t}\left(r_{1}\right)\right|$ & 99.47 & 99.52 & 99. & 98.45 & 65.07 & 96 & 92 & 82.80 \\
\hline$\left|t\left(\hat{\rho}_{k}\right)\right|$ & 99.43 & 99.44 & 99.82 & 98.15 & 64.76 & 99.07 & & 81.09 \\
\hline$\left|\bar{t}\left(\hat{\rho}_{k}\right)\right|$ & 99.45 & 99.47 & 99.85 & 98.44 & 67.80 & 99.12 & 91.64 & 81.68 \\
\hline Sample size: $n=10$ & \multicolumn{8}{|c|}{ Asymptotic tests and bounds } \\
\hline$E_{11}$ & 99.03 & 99.07 & 98.80 & 80.69 & 30.75 & 98.89 & & 79.82 \\
\hline st bol & 9 & 99.11 & 98.84 & 81.06 & 30.77 & 98.91 & & 79.82 \\
\hline & \multicolumn{8}{|c|}{ Tests based on global size correction } \\
\hline$\left|t\left(r_{1}\right)\right|$ & 97.53 & 97.72 & 98.20 & 83.14 & 48.51 & 97.25 & 88.40 & 77.84 \\
\hline$\left|\tilde{t}\left(r_{1}\right)\right|$ & 97.53 & 97.72 & 98.20 & & & & & 77.84 \\
\hline & & 97.04 & & & & & & 76.48 \\
\hline$\left|\bar{t}\left(\hat{\rho}_{k}\right)\right|$ & 97.29 & 97.42 & 97.95 & 83.43 & 50.30 & 96.89 & 87.37 & 77.01 \\
\hline & \multicolumn{8}{|c|}{ Tests based on model-specific size correction } \\
\hline$\left|t\left(r_{1}\right)\right|$ & 99.83 & 99.77 & 99.85 & 98.51 & 64.65 & 99.75 & 95.54 & 83.19 \\
\hline$\left|\tilde{t}\left(r_{1}\right)\right|$ & 99.83 & 99.77 & 99.85 & 98.51 & 64.65 & 99.75 & 95.54 & 83.19 \\
\hline$\left|t\left(\hat{\rho}_{k}\right)\right|$ & 99.83 & 99.74 & 99.84 & 98.47 & & & 94.94 & 82.42 \\
\hline$\left|\bar{t}\left(\hat{\rho}_{k}\right)\right|$ & 99.83 & 99.76 & 99.85 & 98.50 & 66.59 & 99.73 & 95.22 & 82.70 \\
\hline
\end{tabular}


Table C.17. Empirical powers of serial dependence tests at level $\alpha=0.05$

$$
X_{t}=0.9 X_{t-1}+u_{t}, v_{t} \sim \mathrm{N}(0,1)
$$

\begin{tabular}{|c|c|c|c|c|c|c|c|c|}
\hline Sample size: $n=30$ & \multicolumn{8}{|c|}{ Asymptotic tests and bounds } \\
\hline Test $\backslash$ Model & M1 & M2 & M3 & M4 & M5 & M6 & M7 & M8 \\
\hline$E_{11}$ & 97.94 & 97.95 & 99.71 & 80.64 & 19.45 & 90.06 & 83.83 & 84.70 \\
\hline Best $\mathrm{b}$ & 98.20 & 98.18 & 99.76 & 81.25 & 19.45 & 90.46 & 84.21 & 85.06 \\
\hline & \multicolumn{8}{|c|}{ Tests based on global size correction } \\
\hline$\left|t\left(r_{1}\right)\right|$ & 2.69 & 97.09 & 99.61 & 77.68 & 40.57 & 85.59 & 79.63 & 79.43 \\
\hline$\left|\tilde{t}\left(r_{1}\right)\right|$ & 2.69 & 97.09 & 99.61 & 77.68 & 40.57 & 85.59 & & 79.43 \\
\hline & 0.15 & 90.72 & 98.74 & 70.18 & 40.76 & 75.99 & 71.37 & $71.4 \mathrm{C}$ \\
\hline $\bar{t}\left(\hat{\rho}_{k}\right)$ & 5.21 & 93.59 & 99.13 & 76.44 & 47.30 & 79.35 & 73.70 & 73.76 \\
\hline & \multicolumn{8}{|c|}{ Tests based on model-specific size correction } \\
\hline$\left|t\left(r_{1}\right)\right|$ & .65 & 99.95 & 100 & 91.71 & 40.57 & 91.95 & 84.29 & 83.85 \\
\hline$\left|\tilde{t}\left(r_{1}\right)\right|$ & 65 & 99.95 & 100 & 91 & 40.57 & 91.95 & & 83.85 \\
\hline$\left|t\left(\hat{\rho}_{k}\right)\right|$ & .52 & 99.71 & 100 & 87.32 & 40.76 & 85.47 & 77.68 & 77.46 \\
\hline$\left|\bar{t}\left(\hat{\rho}_{k}\right)\right|$ & 9.06 & 99.84 & 100 & 90.09 & 47.30 & 87.62 & 79.27 & 79.06 \\
\hline Sample size: $n=60$ & \multicolumn{8}{|c|}{ Asymptotic tests and bounds } \\
\hline$E_{1}$ & 100 & 100 & 100 & 81.76 & 18.45 & 98.44 & 86.29 & 85.85 \\
\hline Best b & 1 & 100 & 100 & 82.36 & 18.45 & 98.53 & 86.55 & 86.23 \\
\hline & \multicolumn{8}{|c|}{ Tests based on global size correction } \\
\hline$\left|t\left(r_{1}\right)\right|$ & .07 & 99.53 & 99.97 & 77.97 & 38.98 & 92.28 & 80.46 & 80.16 \\
\hline$\left|\tilde{t}\left(r_{1}\right)\right|$ & 9.07 & 99.53 & 99.97 & & 38 & 92.28 & & 80.16 \\
\hline$\left|t\left(\hat{\rho}_{k}\right)\right|$ & 7.44 & 98.53 & 99.87 & & 39 & 87.78 & & 75.68 \\
\hline$\left|\bar{t}\left(\hat{\rho}_{k}\right)\right|$ & 3.08 & 98.83 & 99.88 & 78.13 & 42.55 & 88.83 & 77.40 & 76.55 \\
\hline & \multicolumn{8}{|c|}{ Tests based on model-specific size correction } \\
\hline$\left|t\left(r_{1}\right)\right|$ & & 100 & 10 & 92 & 39.31 & 97.80 & 84.95 & 84.35 \\
\hline$\left|\tilde{t}\left(r_{1}\right)\right|$ & 10 & 100 & 10 & 07 & 39. & 97.80 & 8 & 84.35 \\
\hline$\left|t\left(\hat{\rho}_{k}\right)\right|$ & 10 & 100 & 10 & 90 & 40.00 & 96.26 & 81 & 81.11 \\
\hline$\left|\bar{t}\left(\hat{\rho}_{k}\right)\right|$ & & 100 & 100 & 91.81 & 42.82 & 96.68 & 82.35 & $81.6 \mathrm{c}$ \\
\hline Sample size: $n=1$ & \multicolumn{8}{|c|}{ Asymptotic tests and bounds } \\
\hline$E_{11}$ & 100 & 100 & 100 & & 18.88 & 99.80 & 87.41 & 86.00 \\
\hline Best b & & 100 & 100 & 82.48 & 18.88 & 99.83 & 87.65 & 86.34 \\
\hline & \multicolumn{8}{|c|}{ Tests based on global size correction } \\
\hline$\left|t\left(r_{1}\right)\right|$ & 9.94 & 99.99 & 99.98 & 79.05 & 39.98 & 96.89 & 80.91 & 80.02 \\
\hline$\left|\tilde{t}\left(r_{1}\right)\right|$ & & 99.99 & 99.98 & & & 96.89 & 80.91 & 80.02 \\
\hline$\left|t\left(\hat{\rho}_{k}\right)\right|$ & & 99.94 & 99.98 & & & 95.08 & & 77.62 \\
\hline$\left|\bar{t}\left(\hat{\rho}_{k}\right)\right|$ & 9 & 99.97 & 99.98 & 79.33 & 42.34 & 95.48 & 78.57 & 78.03 \\
\hline & \multicolumn{8}{|c|}{ Tests based on model-specific size correction } \\
\hline$\left|t\left(r_{1}\right)\right|$ & 100 & 100 & 100 & 92.11 & 39.98 & 99.68 & 85.52 & 83.93 \\
\hline$\left|\tilde{t}\left(r_{1}\right)\right|$ & 100 & 100 & 100 & 92.11 & 39.98 & 99.68 & 85.52 & 83.93 \\
\hline$\left|t\left(\hat{\rho}_{k}\right)\right|$ & 100 & 100 & 10 & 91.54 & 40.43 & 99.38 & 83.70 & 82.19 \\
\hline$\left|\bar{t}\left(\hat{\rho}_{k}\right)\right|$ & 100 & 100 & 100 & 92.19 & 42.34 & 99.48 & 84.06 & 82.57 \\
\hline
\end{tabular}


Table C.18. Empirical powers of serial dependence tests at level $\alpha=0.05$

$$
X_{t}=0.9 X_{t-1}+u_{t}, v_{t} \sim \text { Cauchy }
$$

\begin{tabular}{|c|c|c|c|c|c|c|c|c|}
\hline Sample size: $n=30$ & \multicolumn{8}{|c|}{ Asymptotic tests and bounds } \\
\hline Test $\backslash$ Model & M1 & M2 & M3 & M4 & M5 & M6 & M7 & M8 \\
\hline$E_{11}$ & 94.39 & 94.67 & 97.09 & 84.16 & 35.89 & 94.37 & 90.42 & 89.65 \\
\hline Best & 4.55 & 94.92 & 97.13 & 84.56 & 35.95 & 94.58 & 90.81 & 89.97 \\
\hline & \multicolumn{8}{|c|}{ Tests based on global size correction } \\
\hline$\left|t\left(r_{1}\right)\right|$ & 93.46 & 94.40 & 97.42 & 88.32 & 56.06 & 93.38 & 88.42 & 86.01 \\
\hline$\left|\tilde{t}\left(r_{1}\right)\right|$ & 93.46 & 94.40 & 97.42 & 88.32 & & & 2 & 86.01 \\
\hline & 6.36 & 87.83 & 94.99 & 83.62 & 54.99 & 86.57 & 81.97 & 80.07 \\
\hline $\mid \bar{t}(\hat{r}$ & 9.35 & 90.57 & 96.19 & 87.38 & 61.06 & 89.27 & 84.20 & 81.78 \\
\hline & \multicolumn{8}{|c|}{ Tests based on model-specific size correction } \\
\hline$\left|t\left(r_{1}\right)\right|$ & 99 & 99.09 & 99.63 & 98.88 & 71.44 & 98.30 & 92.65 & 88.98 \\
\hline$\left|\tilde{t}\left(r_{1}\right)\right|$ & .99 & 99.09 & 99.63 & 98.88 & & & 92 & 88.98 \\
\hline$\left|t\left(\hat{\rho}_{k}\right)\right|$ & 98.28 & 98.41 & 99.40 & 98.26 & 71.21 & 96.99 & 88 & 84.87 \\
\hline$\left|\bar{t}\left(\hat{\rho}_{k}\right)\right|$ & 98.59 & 98.64 & 99.47 & 98.65 & 77.24 & 97.53 & 89.86 & 85.98 \\
\hline Sample size: $n=60$ & \multicolumn{8}{|c|}{ Asymptotic tests and bounds } \\
\hline$E_{11}$ & 99.09 & 99.15 & 99.32 & 87.28 & 35.00 & 99.04 & 96.03 & 90.32 \\
\hline Best bc & 9 & 99.18 & 99.37 & 87.48 & 35.10 & 99.07 & 96.28 & 90.52 \\
\hline & \multicolumn{8}{|c|}{ Tests based on global size correction } \\
\hline$\left|t\left(r_{1}\right)\right|$ & 97.93 & 98.15 & 98.91 & 90.44 & 55.43 & 97.90 & 92.95 & 86.61 \\
\hline$\left|\tilde{t}\left(r_{1}\right)\right|$ & 97.93 & 98.15 & 98.91 & 90 & & & & 86.61 \\
\hline$\left|t\left(\hat{\rho}_{k}\right)\right|$ & 97.02 & 97.44 & 98.39 & 89 & & 97 & & 83.31 \\
\hline$\left|\bar{t}\left(\hat{\rho}_{k}\right)\right|$ & 97.43 & 97.68 & 98.58 & 90.46 & 58.09 & 97.34 & 91.07 & 83.98 \\
\hline & \multicolumn{8}{|c|}{ Tests based on model-specific size correction } \\
\hline$\left|t\left(r_{1}\right)\right|$ & 99.68 & 99.62 & 99.88 & 99.34 & 70.70 & 99.62 & 96.31 & 89.96 \\
\hline$\left|\tilde{t}\left(r_{1}\right)\right|$ & 99.68 & 99.62 & 99. & 99. & 70.70 & 99 & & 89.96 \\
\hline$\left|t\left(\hat{\rho}_{k}\right)\right|$ & 99.58 & 99.57 & 99.88 & 99.23 & 70.87 & 99.52 & 94 & 87.73 \\
\hline$\left|\bar{t}\left(\hat{\rho}_{k}\right)\right|$ & 99.60 & 99.59 & 99.88 & 99.35 & 74.26 & 99.54 & 95.35 & 88.13 \\
\hline Sample size: $n=10$ & \multicolumn{8}{|c|}{ Asymptotic tests and bounds } \\
\hline$E_{11}$ & 99.60 & 99.70 & 99.80 & 88. & 34.89 & 99.65 & 98 & 90.24 \\
\hline st bou & 99 & 99.71 & 99.81 & 88.60 & 34.98 & 99.65 & & \\
\hline & \multicolumn{8}{|c|}{ Tests based on global size correction } \\
\hline$\left|t\left(r_{1}\right)\right|$ & 99.03 & 99.01 & 99.35 & 90.69 & 56.30 & 99.04 & 95.66 & 86.61 \\
\hline$\left|\tilde{t}\left(r_{1}\right)\right|$ & 99.03 & 99.01 & & & & & & 86.61 \\
\hline & & 98.84 & 99. & 90. & & & & 84. \\
\hline$\left|\bar{t}\left(\hat{\rho}_{k}\right)\right|$ & 98.94 & 98.93 & 99.26 & 90.91 & 57.93 & 98.89 & 94.94 & 85.17 \\
\hline & \multicolumn{8}{|c|}{ Tests based on model-specific size correction } \\
\hline$\left|t\left(r_{1}\right)\right|$ & 99.87 & 99.79 & 99.98 & 99.27 & 71.04 & 99.89 & 98.24 & 89.71 \\
\hline$\left|\tilde{t}\left(r_{1}\right)\right|$ & 99.87 & 99.79 & 99.98 & 99.27 & 71.04 & 99.89 & 98.24 & 89.71 \\
\hline$\left|t\left(\hat{\rho}_{k}\right)\right|$ & 99.84 & 99.78 & 99.96 & 99.24 & 70.90 & 99.87 & 97.84 & 88.49 \\
\hline$\left|\bar{t}\left(\hat{\rho}_{k}\right)\right|$ & 99.85 & 99.78 & 99.98 & 99.28 & 72.87 & 99.88 & 97.95 & 88.76 \\
\hline
\end{tabular}




\section{References}

Anderson, T. W. (1971), The Statistical Analysis of Time Series, John Wiley \& Sons, New York.

Balke, N. S. and Gordon, R. J. (1986), Historical data, in R. J. Gordon, ed., 'The American Business Cycle: Continuity and Change', University of Chicago Press, Chicago, Illinois, pp. 781-850.

Brockwell, P. J. and Davis, R. A. (1991), Time Series: Theory and Methods, second edn, SpringerVerlag, New York.

Campbell, B. and Dufour, J.-M. (1997), 'Exact nonparametric tests of orthogonality and random walk in the presence of a drift parameter', International Economic Review 38, 151-173.

Dufour, J.-M. (1981), 'Rank tests for serial dependence', Journal of Time Series Analysis 2, 117128.

Dufour, J.-M. (1990), 'Exact tests and confidence sets in linear regressions with autocorrelated errors', Econometrica 58, 475-494.

Dufour, J.-M. (2003), 'Identification, weak instruments and statistical inference in econometrics', Canadian Journal of Economics 36(4), 767-808.

Dufour, J.-M. and Hallin, M. (1991), 'Nonuniform bounds for nonparametric $t$ tests', Econometric Theory 7, 253-263.

Dufour, J.-M. and Hallin, M. (1992), 'Simple exact bounds for distributions of linear signed rank statistics', Journal of Statistical Planning and Inference 31, 311-333.

Dufour, J.-M. and Hallin, M. (1993), 'Improved Eaton bounds for linear combinations of bounded random variables, with statistical applications', Journal of the American Statistical Association 88, 1026-1033.

Dufour, J.-M. and King, M. L. (1991), 'Optimal invariant tests for the autocorrelation coefficient in linear regressions with stationary or nonstationary AR(1) errors', Journal of Econometrics 47, 115-143.

Dufour, J.-M. and Kiviet, J. F. (1998), 'Exact inference methods for first-order autoregressive distributed lag models', Econometrica 66, 79-104.

Dufour, J.-M. and Roy, R. (1985), 'Some exact results on sample autocorrelations and tests of randomness', Journal of Econometrics 29, 257-273. Corrigendum 41(1989), 279-281.

Eaton, M. L. (1970), 'A note on symmetric Bernoulli random variables', The Annals of Statistics 41, 1223-1226.

Edelman, D. (1986), 'Bounds for a nonparametric $t$ table', Biometrika 73, 242-243. 
Edelman, D. (1990), 'An inequality of optimal order for the tail probabilities of the $t$-statistic under symmetry', Journal of the American Statistical Association 85, 120-122.

Efron, B. (1969), 'Student's $t$-test under symmetry conditions', Journal of the American Statistical Association 81, 461-470.

Hallin, M. and Puri, M. L. (1992), Rank tests for time series analysis: A survey, in D. Brillinger, P. Caines, J. Geweke, E. Parzen, M. Rosenblatt and M. S. Taqqu, eds, 'New Directions in Time Series Analysis, Part I', Vol. 45 of The IMA Volumes in Mathematics and its Applications, Springer-Verlag, New York, pp. 111-153.

Hoeffding, W. (1963), 'Probability inequalities for sums of bounded random variables', Journal of the American Statistical Association 58, 13-20.

Imhof, P. J. (1961), 'Computing the distribution of quadratic forms in normal variables', Biometrika 48, 419-426. Corrigendum 49 (1962), 284.

Koerts, J. and Abrahamse, A. P. J. (1969), On the Theory and Application of the General Linear Model, Rotterdam University Press, Rotterdam, The Netherlands.

Lehmann, E. L. (1986), Testing Statistical Hypotheses, 2nd edition, John Wiley \& Sons, New York.

Lehmann, E. L. and Stein, C. (1949), 'On the theory of some non-parametric hypotheses', Annals of Mathematical Statistics 20, 28-45.

Pinelis, I. (1994), 'Extremal probabilistic problems and Hotelling's $T^{2}$ test under symmetry condition', The Annals of Statistics 22(1), 357-368.

Pratt, J. W. and Gibbons, J. D. (1981), Concepts of Nonparametric Theory, Springer-Verlag, New York.

van Beek, P. (1972), 'An application of Fourier methods to the problem of sharpening the BerryEsséen inequality', Zeitschrift für Wahrscheinlichkeitstheorie und verwandte Gebiete 23, 187196.

Whistler, D., White, K. J., Wong, S. D. and Bates, D. (2001), SHAZAM Version 9 User's Reference Manual, Northwest Econometrics, Vancouver, Canada.

Zolotarev, V. M. (1965), 'On the closeness of the distributions of two sums of independent random variables', Theory of Probability and Its Applications 10, 472-479. 